\title{
Estimação de medidas de risco utilizando modelos CAViaR e CARE.
}

\author{
Francyelle de Lima e Silva \\ DISSERTAÇÃO APRESENTADA \\ $\mathrm{AO}$ \\ Instituto De Matemática e Estatística \\ DA \\ UniversidAdE DE SÃO PAUlO \\ PARA \\ OBTENÇÃO DO TÍTULO \\ $\mathrm{DE}$ \\ Mestre EM Cî̂nCIAS
}

Programa: Estatística

Orientadora: Prof ${ }^{\mathrm{a}}$. Dr ${ }^{\mathrm{a}}$. Clelia Maria de Castro Toloi

São Paulo, Agosto de 2010 


\title{
Estimação de medidas de risco utilizando modelos CAViaR e CARE.
}

\author{
Este exemplar corresponde à redação \\ final da dissertação devidamente corrigida \\ e defendida por Francyelle de Lima e Silva \\ e aprovada pela Comissão Julgadora.
}

Banca Examinadora:

- Prof ${ }^{\mathrm{a}}$. Dr ${ }^{\mathrm{a}}$. Clelia Maria de Castro Toloi (orientadora) - IME-USP.

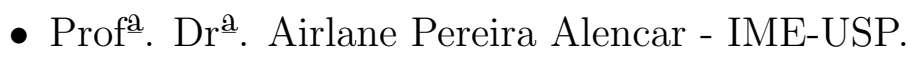

- Prof ${ }^{\mathrm{a}}$. Dr ${ }^{\mathrm{a}}$. Adriana Bruscato Bortoluzzo - INSPER. 


\section{Agradecimentos}

Inicialmente, agradeço aos meus pais, Ionice e Aparecido, que sempre me apoiaram e me orientaram em todas as etapas da minha vida, ajudando a fazer com que meus objetivos pudessem ser alcançados. Se não fosse por eles, a realização deste trabalho e a minha vinda a São Paulo não seriam possíveis.

A Professora Clélia Maria de Castro Toloi por me orientar e me ajudar, além de me incentivar em cada etapa do trabalho.

Agradeço aos meus queridos amigos cearenses João Ítalo e Rafael Paixão pelo companheirismo e discussões sobre aspectos computacionas e estatísticos, além dos grandes momentos de alegria e descontração.

As minhas grandes amigas Patrícia e Liliane pela contribuição e ajuda durante todo o mestrado.

A todas as minhas amigas do sul, pelos momentos alegres que tornaram meus dias em São Paulo especiais e inesquecíveis.

E finalmente, a todos os meus amigos e colegas que contribuíram direta e indiretamente para a elaboração e conclusão deste trabalho, além das pessoas que convivem comigo e contribuem a cada dia pra que eu me torne uma pessoa melhor. 


\section{Resumo}

Neste trabalho são definidos, discutidos e estimados o Valor em Risco e o Expected Shortfall. Estas são medidas de Risco Financeiro de Mercado muito utilizadas por empresas e investidores para o gerenciamento do risco, aos quais podem estar expostos.

O objetivo foi apresentar e utilizar vários métodos e modelos para a estimação dessas medidas e estabelecer qual o modelo mais adequado dentro de determinados cenários.

Palavras-chave: Quantil, Expectil, Teoria dos Valores Extremos, Valor em Risco, Expected Shortfall, Modelos CAViaR, Modelos CARE. 


\section{Abstract}

In this work Value at Risk and Expected Shortfall are defined, discussed and estimated . These are measures heavily used in Financial Market Risk, in particular by companies and investors to manage risk, which they may be exposed.

The aim is to present and use several methods and models for estimating those measures and to establish which model is most appropriate in certain scenarios.

Keywords: Quantile, Expectile, Extreme Value Theory, Value at Risk, Expected Shortfall, CAViaR Models, CARE Models. 


\section{Sumário}

Lista de Figuras $\quad$ xiii

Lista de Tabelas $\quad$ xv

1 Introdução 1

2 VaR - Valor em Risco $\quad 5$

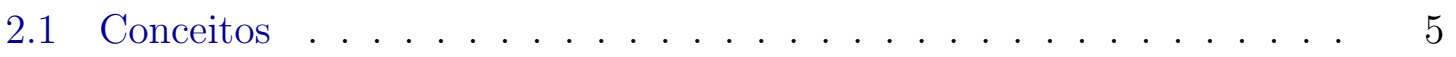

2.2 VaR Usando a Distribuição Normal . . . . . . . . . . . . . . . 8

2.3 VaR Usando a Distribuição t-Student . . . . . . . . . . . . . . . . 10

2.4 VaR Usando a Distribuição t-Student Generalizada Assimétrica . . 12

3 TVE - Teoria dos Valores Extremos $\quad 19$

3.1 Conceitos . . . . . . . . . . . . . . . . . . . . . . . 19

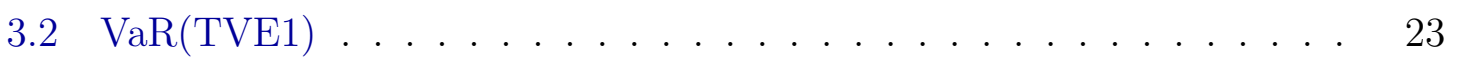

$3.3 \quad \operatorname{VaR}(\mathrm{TVE} 2) \ldots \ldots \ldots \ldots \ldots$

3.4 Verificação dos Métodos para o VaR . . . . . . . . . . . . 28

4 CAViaR - Valor em Risco Autorregressivo Condicional 31

4.1 Conceitos . . . . . . . . . . . . . . . . . . 31

4.2 Especificações dos Modelos CAViaR . . . . . . . . . . . . . . . 32

4.2 .1 Valor Absoluto Simétrico: . . . . . . . . . . . . . . . . . 32 
4.2 .2 Inclinação Assimétrica: . . . . . . . . . . . . . . . . . 33

$4.2 .3 \quad \operatorname{GARCH}(1,1)$ Indireto: . . . . . . . . . . . . . . . . . . 33

4.2.4 Adaptativo: . . . . . . . . . . . . . . . . . . . 33

4.2.5 Adaptativo Simétrico Proporcional: . . . . . . . . . . . . . 34

4.2.6 Valor Absoluto Assimétrico: . . . . . . . . . . . . . . . . 34

4.2.7 $\operatorname{AR}(1)-\operatorname{GARCH}(1,1)$ indireto: . . . . . . . . . . . . 34

4.3 Estimação do Modelo . . . . . . . . . . . . . . . . . . . . . . . 36

4.3 .1 Regressão Quantílica . . . . . . . . . . . . . . . . . 36

4.4 Teste de Ajuste dos Modelos . . . . . . . . . . . . . . . . . . 40

5 CARE - Expectil Autorregressivo Condicional 45

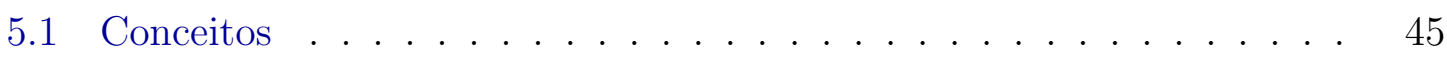

5.2 Expectil . . . . . . . . . . . . . . . . . . 46

5.3 Especificações dos modelos CARE . . . . . . . . . . . . . . . . . . 49

5.3.1 Valor Absoluto Simétrico: . . . . . . . . . . . . . . . . . . 49

5.3 .2 Inclinação Assimétrica: . . . . . . . . . . . . . . . . . 49

5.3.3 GARCH(1,1) Indireto: . . . . . . . . . . . . . . . . . . 49

5.3 .4 Adaptativo: . . . . . . . . . . . . . . . . . . . . . 49

5.3.5 Adaptativo Simétrico Proporcional: . . . . . . . . . . . . . . . 49

5.3.6 Valor Absoluto Assimétrico: . . . . . . . . . . . . . . . . . . 49

5.3.7 $\operatorname{AR}(1)-\operatorname{GARCH}(1,1)$ Indireto: . . . . . . . . . . . . . . 50

5.3 .8 Especificação $[1] \ldots \ldots \ldots \ldots$. . . . . . . . . . . . . . . . 51

5.3 .9 Especificação $[2] \ldots \ldots \ldots \ldots \ldots$. . . . . . . . . . . . . 52

5.3 .10 Especificação $[3] \ldots \ldots \ldots \ldots$. . . . . . . . . . . . . . 52

5.3 .11 Especificação $[4] \ldots \ldots \ldots \ldots \ldots$

5.4 Estimação do Modelo . . . . . . . . . . . . . . . . . . . . . . . . 53

5.5 Teste de Ajuste dos Modelos . . . . . . . . . . . . . . . . 55

$\begin{array}{lll}6 & \text { ES - Expected Shortfall } & \mathbf{6 1}\end{array}$

6.1 Conceitos . . . . . . . . . . . . . . . . . . . 61 
6.2 ES Usando a Distribuição Normal . . . . . . . . . . . . . . . . . . 63

6.3 ES Usando a Distribuição t-Student . . . . . . . . . . . . . . . . . . . 65

6.4 ES Utilizando Expectil . . . . . . . . . . . . . . . . . 66

6.5 ES Usando Teoria dos Valores Extremos . . . . . . . . . . . . . 68

6.5.1 $\mathrm{ES}(\mathrm{TVE} 1) \ldots \ldots \ldots \ldots . \ldots . \ldots . \ldots 68$

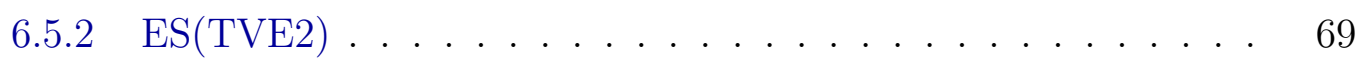

6.6 Verificação dos Métodos e Modelos para o ES . . . . . . . . . . . 70

7 Aplicações $\quad 73$

7.1 Descrição da Série . . . . . . . . . . . . . . . . . . . . . . 73

7.2 Cálculo do VaR . . . . . . . . . . . . . . . . . . 76

7.2.1 Abordagem Econométrica . . . . . . . . . . . 76

7.2 .2 Teoria dos Valores Extremos . . . . . . . . . . . . . . 79

7.2.3 Modelos CAViaR .................. 83

7.2.4 Modelos CARE . . . . . . . . . . . . . . 90

7.3 Calculo do ES . . . . . . . . . . . . . . . . . . . 101

7.3.1 Abordagem Econométrica . . . . . . . . . . . . . . . 101

7.3.2 Teoria dos Valores Extremos . . . . . . . . . . . . . . 102

7.3.3 Modelos CARE . . . . . . . . . . . . . . 103

8 Conclusões 109

A Distribuição T-Student Generalizada Assimétrica. 113

B Medidas de Risco $\quad 115$

C Método da Aceitação-Rejeição 119

$\begin{array}{ll}\text { D Teste Bootstrap } & 121\end{array}$

E Ajuste do Modelo ARMA-TARCH 123 
Referências Bibliográficas 


\section{Lista de Abreviaturas}

$\begin{array}{ll}\text { CARE } & \text { Expectil Autorregressivo Condicional. } \\ \text { CAViaR } & \text { Valor em Risco Autorregressivo Condicional. } \\ \text { CQ } & \text { Critério Quantílico. } \\ \text { DGP } & \text { Distribuição Generalizada de Pareto. } \\ \text { ES } & \text { Expected Shortfall. } \\ \text { f.d.p. } & \text { Função Densidade de Probabilidade. } \\ \text { g.l. } & \text { graus de liberdade } \\ \text { GVE } & \text { Generalizada de Valores Extremos } \\ \text { IBOVESPA } & \text { Índice da Bolsa de Valores de São Paulo. } \\ \text { MQA } & \text { Mínimos Quadrados Assimétricos. } \\ \text { QD } & \text { Quantis Dinâmicos. } \\ \text { QD am } & \text { Quantis Dinâmicos Amostrais. } \\ \text { QD } D_{\text {pam }} & \text { Quantis Dinâmicos pós-amostrais. } \\ \text { RQ } & \text { Regressão Quantílica. } \\ \text { tsga } & \text { t-Student Generalizada Assimétrica. } \\ \text { TVE } & \text { Teoria dos Valores Extremos. } \\ \text { v.a. } & \text { Variável Aleatória. } \\ \text { VaR } & \text { Valor em Risco. }\end{array}$




\section{Lista de Figuras}

7.1 Gráficos da série de IBOVESPA e da série de log-retornos. . . . . . . 74

7.2 Gráficos do histograma com densidade normal ajustada e QQ-Plot dos $\log$-retornos. . . . . . . . . . . . . . . . . . 74

7.3 Estimativa da volatilidade para os retornos do IBOVESPA. . . . . . 76

7.4 QQ-Plot dos resíduos padronizados. . . . . . . . . . . 77

7.5 Estimativas para o VaR, $\operatorname{com} \theta=0,05$ e $\theta=0,95 \ldots \ldots \ldots \ldots 79$

7.6 Gráfico da Média dos Excessos para os log-retornos. . . . . . . . . 80

7.7 Estimativas para o VaR, $\operatorname{com} \theta=0,05$ e $\theta=0,95$ utilizando TVE. . $\quad 81$

7.8 Gráfico da Média dos Excessos para os Resíduos Padronizados. . . . . 81

7.9 Estimativas para o VaR, $\operatorname{com} \theta=0,05$ e $\theta=0,95$ utilizando TVE. . $\quad 82$

7.10 Gráficos de VaR estimados através dos modelos CAViaR. . . . . . . 85

7.11 Gráfico do $\theta$ - quantil e $\tau$-expectil obtidos para a série de log-retornos. 90

7.12 Gráficos de VaR estimados através dos modelos CARE. . . . . . . . . 92

7.13 Estimativas para o VaR utilizando modelos CARE . . . . . . . . . 98

7.14 Estimativas para o VaR e ES, $\operatorname{com} \theta=0,05 \ldots \ldots \ldots \ldots \ldots$

7.15 Estimativas para o VaR e ES, $\operatorname{com} \theta=0,05$ utilizando TVE. . . . . . 102

7.16 Estimativas para o VaR e ES, $\operatorname{com} \theta=0,05$ utilizando TVE. . . . . . 103

7.17 Gráficos de VaR e ES estimados através dos modelos CARE. . . . . . 105

E.1 Ajuste do modelo AR(10)-TARCH(1,1) . . . . . . . . . . . . 124 


\section{Lista de Tabelas}

7.1 Estatísticas da série de log-retornos do IBOVESPA. . . . . . . . . 75

7.2 Valores do Teste de Kolmogorov-Smirnov . . . . . . . . . . . . . 78

7.3 Valores Estimados para os Parâmetros das DGP ajustadas. . . . . . . 82

7.4 Número de Excessos e Proporção para os métodos adotados. . . . . . 82

7.5 Estimativas e Estatísticas dos Parâmetros Estimados Utilizando as Especificações do Modelo CAViaR. . . . . . . . . . . . . . . . 86

7.6 Estimativas e Estatísticas dos Parâmetros Estimados Utilizando as Especificações do Modelo CAViaR. . . . . . . . . . . . . . . . 87

7.7 Estimativas e Estatísticas dos Parâmetros Estimados Utilizando as Especificações do Modelo CARE. . . . . . . . . . . . . . . . . . . . 93

7.8 Estimativas e Estatísticas dos Parâmetros Estimados Utilizando as Especificações do Modelo CARE. . . . . . . . . . . . . . . . . . . . 94

7.9 Estimativas e Estatísticas dos Parâmetros Estimados Utilizando a Especificação 3 do Modelo CARE. . . . . . . . . . . . . . . . . . . 99

7.10 Estimativas e Estatísticas dos Parâmetros Estimados Utilizando a Especificação 4 do Modelo CARE. . . . . . . . . . . . . . . . 100

7.11 Hits Amostrais e Pós-amostrais e Valor do MQA. . . . . . . . . . . . 101

7.12 Estatísticas do Teste Bootstrap para ES utilizando Abordagem Econométrica, TVE e modelos CARE. . . . . . . . . . . . . . . 106 


\section{Capítulo 1}

\section{Introdução}

No mercado financeiro, existem três tipos de risco aos quais as empresas estão expostas: operacional, estratégico e financeiro. Este último pode ainda ser subdividido em: operacional, de crédito, de liquidez, legal e de mercado.

O trabalho apresenta somente medidas de riscos financeiros de mercado, que estão associadas às possíveis perdas ou ganhos potenciais de investimentos (posições financeiras) de acordo com o comportamento do mercado.

Os investimentos citados no trabalho são referências feitas às posições financeiras definidas como vendidas ou compradas.

No Capítulo 2, de acordo com Morettin (2008), é definido e apresentado o Valor em Risco (VaR), além dos métodos de estimação pela abordagem econométrica, supondo distribuição Normal, t-Student e a distribuição t-Student Generalizada Assimétrica aos erros. A distribuição assimétrica proposta tem densidade seguindo as idéias de Fernándes e Steel (1998).

No Capítulo 3 são apresentados os métodos de estimação para o VaR utilizando abordagem econométrica juntamente com a Teoria dos Valores Extremos (TVE), a partir da escolha de um limiar apropriado, não considerando apenas máximos de blocos.

Após a estimação do VaR, visando qual o método que fornece medidas de risco 
mais precisas e adequadas em cada cenário, apresenta-se também o método de verificação para esta medida.

Ao longo do trabalho, foi possível notar que o VaR apresenta certas fragilidades, como por exemplo a suposição inadequada de distribuição dos erros, resultando em estimativas imprecisas. Sendo assim, no Capítulo 4 são detalhados e apresentados os modelos de Valor em Risco Autorregressivo Condicional (CAViaR) propostos por Engle e Manganelli (2004), que ao invés de modelar toda a distribuição dos retornos, modela diretamente seus quantis. São comentados e apresentados todos os testes para fins de verificação, seleção e adequação dos modelos adotados.

No Capítulo 5 são apresentados os modelos Expectil Autorregressivo Condicional (CARE) propostos por Taylor (2008), que também são utilizados para a estimação do VaR. Estes modelos possuem estruturas semelhantes aos modelos CAViaR, porém a diferença está no fato de possuir estimativas mais influenciadas pela magnitude dos retornos. Visando discutir melhor as propriedades dos modelos CARE, Kuan, Yeh e Hsu (2009) propõem novas especificações, cujas propriedades assintóticas e testes são apresentados ao longo do Capítulo 5.

O VaR, como comentado, é uma das medidas de risco mais utilizadas no mercado para fim de controle e gerenciamento de risco, mas que possui fragilidades, como por exemplo não ser uma medida de risco coerente. Sendo assim, surge a medida denominada como Expected Shortfall (ES), apresentada no Capítulo 6, fornecendo informações sobre os retornos que estão acima ou abaixo do Valor em Risco, dependendo da posição, além de ser uma medida de risco coerente. O trabalho apresenta os métodos de estimação para o ES considerando os modelos CARE propostos por Taylor (2008), além da abordagem econométrica supondo algumas distribuições aos erros e o uso da TVE detalhada no Capítulo 3. O método de verificação e adequação dos modelos também é apresentado.

No Capítulo 7 são estimados todos os modelos para a mensuração do VaR e ES, além do cálculo de testes para fins de seleção e adequação dos modelos. Todas as aplicações foram feitas para a série de Índices da Bolsa de Valores de São Paulo 
(IBOVESPA), constituída de valores no período de 04/07/1994 a 11/02/2010.

De forma geral, o que se objetivou na elaboração do trabalho não foi definir e nem escolher uma medida de risco que seja considerada como a mais correta, mas apresentar vários modelos e métodos utilizando os mais distintos conceitos e abordagens, resultando em opções para a obtenção de uma medida de risco adequada em determinados cenários, já que o comportamento das observações influencia diretamente na estimação e verificação dos modelos. 


\section{Capítulo 2}

\section{VaR - Valor em Risco}

\subsection{Conceitos}

De acordo com Morettin (2008), uma posição financeira comprada (ou "long") significa possuir determinado ativo (ou carteira de ativos). Neste caso, há uma perda se há diminuição no valor do ativo. Posição financeira vendida (ou "short") envolve vender um ativo que não se possui. Esta operação é realizada alugando-se um ativo, isto é, em data futura, o vendedor é obrigado a comprar exatamente o mesmo número de cotas ou ações alugadas (e não o valor em moeda), para pagar o débito. Como o pagamento é em cotas ou ações, o vendedor ganha com a queda do preço do ativo. Assim, pode-se notar a importância de calcular, a um dado horizonte, com dada probabilidade, o comportamento da variação dos preços de ativos.

Um dos métodos muito utilizado na mensuração do risco é o Valor em Risco (VaR), traduzido do inglês Value at Risk. Este método utiliza técnicas estatísticas comumente usadas pelos principais bancos e instituições financeiras do mundo, para o cálculo e controle de riscos de mercado.

O VaR envolve o cálculo da volatilidade de um ativo financeiro ou de uma carteira de instrumentos financeiros, dando uma medida do grau de incerteza sobre retornos líquidos futuros. Ele mede a variação potencial máxima do valor de um ativo (ou 
carteira de ativos), sobre um período pré-fixado, com dada probabilidade, ou seja, o mínimo que se pode perder, com probabilidade $\theta$, sobre um horizonte $h, h>0$ fixado. Do ponto de vista de uma empresa, o VaR é uma medida de perda associada a um evento extremo, sob condições normais de mercado.

Assim, dado um nível de confiança $\theta \in(0,1)$, o VaR de uma posição vendida de um portfólio é o menor valor $l$ tal que a probabilidade da perda ou lucro $\Delta P(h)$ sobre um horizonte $h$ exceder $l$ é menor ou igual a $(1-\theta)$, em que:

$$
\Delta P(h)=P(t+h)-P(t)
$$

é a variação do valor do ativo entre dois instantes. Formalmente:

$$
V_{a} R_{t+h}(\theta)=\inf \{l \in \mathbb{R}, P(\Delta P(h)>l) \leq 1-\theta\} .
$$

Já para posição comprada, o VaR é definido como

$$
\operatorname{VaR}_{t+h}(\theta)=\inf \{l \in \mathbb{R}, P(\Delta P(h) \leq l) \leq \theta\} .
$$

Contudo, considerando a função de distribuição acumulada (f.d.a) $F_{h}($.) de $\Delta P(h)$, para o cálculo do $\theta$ - quantil, deseja-se encontrar

$$
\inf \left\{l \in \mathbb{R}, 1-F_{h}(l) \leq 1-\theta\right\}=\inf \left\{l \in \mathbb{R}, F_{h}(l) \geq \theta\right\} \text {. }
$$

Com isso, é possível notar que a definição de VaR coincide com a definição de $\theta$ - quantil em termos da função inversa generalizada da f.d.a $F_{h}($.$) , tanto para$ posições vendidas como para posições compradas.

As medidas de risco são frequentemente medidas em termos das variações dos preços dos ativos.

Seja

$$
\begin{aligned}
R_{t} & =\frac{P(t)-P(t-1)}{P(t-1)}=\frac{\Delta P(t)}{P(t-1)} \Rightarrow R_{t}=\frac{P(t)}{P(t-1)}-1 \Rightarrow \\
1+R_{t} & =\frac{P(t)}{P(t-1)},
\end{aligned}
$$


em que $1+R_{t}$ é chamado de retorno bruto simples. $R_{t}$ é usualmente expresso em porcentagem, relativa ao período de um dia, um mês, um ano, etc. É também chamado de taxa de retorno. Denotando $p_{t}=\log P(t)$, sendo o logarítmo neperiano, define-se o retorno composto continuamente ou simplesmente log-retorno como

$$
r_{t}=\log \frac{P(t)}{P(t-1)}=\log \left(1+R_{t}\right)=p_{t}-p_{t-1} .
$$

Muitas vezes, $r_{t}$ será chamado simplesmente de retorno.

Na prática é preferível trabalhar com retornos, que são livres de escala, do que com preços, pois os retornos possuem propriedades estatísticas que facilitam o ajuste de alguns modelos. É possivel notar também que para um $w$ pequeno, $\log (1+w) \approx w$, do que segue que os retornos simples $R_{t}$ e os log-retornos $r_{t}$ serão em geral valores próximos, assim a suposição de que os log-retornos também medem variações percentuais é válida. Assim, para o cálculo do VaR, utiliza-se log-retornos.

Lembrando que o $\operatorname{VaR}_{\theta}(h)$ de uma posição comprada é um $\theta$ - quantil para um horizonte $h$, a quantia do VaR em unidades monetárias é obtida através da multiplicação do valor da posição financeira pelo VaR dos log-retornos.

Existem várias metodologias para o cálculo do VAR:

a) RiskMetrics, o qual conta com a suposição de que a distribuição condicional dos retornos segue uma distribuição normal, modelando a volatilidade por meio do modelo EWMA("exponentially weighted moving average");

b) Estimação Quantílica, feita a partir dos quantis empíricos dos retornos;

c) Teoria dos Valores Extremos (TVE), em que a estimação é feita a partir de estatísticas de ordem;

d) Abordagem Econométrica, que estima o VaR a partir dos modelos ARMA-GARCH.

Inicialmente, o trabalho apresenta a abordagem econométrica, classificada como um método paramétrico, por assumir algumas distribuições. Serão utilizados modelos lineares da família ARMA para modelar as autocorrelações que algumas séries 
de retornos apresentam e, após a obtenção dos resíduos $a_{t}$ do modelo, ajusta-se um modelo não-linear da família $\mathrm{ARCH}$ para modelar e fazer previsões da volatilidade dos retornos.

Consideraremos o modelo:

$$
r_{t}=\mu_{t}+a_{t} \Rightarrow r_{t}=\mu_{t}+\sigma_{t} \epsilon_{t},
$$

em que $r_{t}$ são os log-retornos da série financeira, $\mu_{t}=E\left\{r_{t} \mid \mathcal{F}_{t-1}\right\}$ a média variando no tempo, $a_{t}=\sigma_{t} \epsilon_{t}$ a parte aleatória, com $\sigma_{t}$ sendo a parte da volatilidade variando com o tempo, ou seja, um modelo heteroscedástico e $\epsilon_{t} \stackrel{i i d}{\sim}(0,1)$ os erros aleatórios, com distribuição $f_{\epsilon}($.$) .$

Como $\mu_{t}$ e $\sigma_{t}$ variam no tempo, é necessário que existam modelos que possam explicar o comportamento dessas medidas a partir de informações passadas, por isso a importância dos modelos ARMA(p,q)-GARCH(r,s), que possuem a seguinte estrutura:

$$
\begin{aligned}
r_{t} & =\phi_{0}+\sum_{i=1}^{p} \phi_{i} r_{t-i}-\sum_{j=1}^{q} \theta_{j} a_{t-j}+a_{t} \\
a_{t} & =\sigma_{t} \epsilon_{t} \\
\sigma_{t}^{2} & =c_{0}+\sum_{i=1}^{r} c_{i} a_{t-i}^{2}+\sum_{j=1}^{s} d_{j} \sigma_{t-j}^{2},
\end{aligned}
$$

em que, sob o modelo (2.3), $\mu_{t}=\phi_{0}+\sum_{i=1}^{p} \phi_{i} r_{t-i}-\sum_{j=1}^{q} \theta_{j} a_{t-j}$, de acordo com (2.4) e $\sigma_{t}$ é modelado de acordo com (2.6).

Utilizando estes modelos, pode se fazer suposições sobre a distribuição dos erros para obter o valor do VaR. A seguir, são apresentadas algumas dessas suposições.

\subsection{VaR Usando a Distribuição Normal}

Supondo

$$
\epsilon_{t} \sim N(0,1)
$$


de acordo com o modelo (2.3), considerando $r_{t}$ variando no tempo, $r_{t} \mid \mathcal{F}_{t-1}$ é definida como uma distribuição elíptica com parâmetros de posição (ou localização) $\mu_{t}$ e escala $\sigma_{t}^{2}\left(\sigma_{t}^{2}>0\right)$.

De fato

$$
\begin{aligned}
E\left\{r_{t} \mid \mathcal{F}_{t-1}\right\} & =E\left\{\left(\mu_{t}+\sigma_{t} \epsilon_{t}\right) \mid \mathcal{F}_{t-1}\right\}=E\left\{\mu_{t} \mid \mathcal{F}_{t-1}\right\}+E\left\{\sigma_{t} \epsilon_{t} \mid \mathcal{F}_{t-1}\right\} \\
& =\mu_{t}+\sigma_{t} E\left\{\epsilon_{t} \mid \mathcal{F}_{t-1}\right\}=\mu_{t} \\
\operatorname{e} \operatorname{Var}\left\{r_{t} \mid \mathcal{F}_{t-1}\right\} & =E\left\{\left(\mu_{t}+\sigma_{t} \epsilon_{t}\right)^{2} \mid \mathcal{F}_{t-1}\right\}-\left\{E\left(r_{t} \mid \mathcal{F}_{t-1}\right)\right\}^{2}= \\
& =E\left\{\left(\mu_{t}^{2}+2 \mu_{t} \sigma_{t} \epsilon_{t}+\sigma_{t}^{2} \epsilon_{t}^{2}\right) \mid \mathcal{F}_{t-1}\right\}-\left\{E\left(r_{t} \mid \mathcal{F}_{t-1}\right)\right\}^{2}= \\
& =E\left\{\mu_{t}^{2} \mid \mathcal{F}_{t-1}\right\}+2 \mu_{t} \sigma_{t} E\left\{\epsilon_{t} \mid \mathcal{F}_{t-1}\right\}+\sigma_{t}^{2} E\left\{\epsilon_{t}^{2} \mid \mathcal{F}_{t-1}\right\}-\mu_{t}^{2}= \\
& =\mu_{t}^{2}+\sigma_{t}^{2}-\mu_{t}^{2}=\sigma_{t}^{2},
\end{aligned}
$$

$\log \mathrm{O}, r_{t} \mid \mathcal{F}_{t-1} \sim N\left(\mu_{t}, \sigma_{t}^{2}\right)$.

De acordo com a definição de VaR, dada em (2.1):

$P\left(r_{t} \mid \mathcal{F}_{t-1}>l\right) \leq(1-\theta) \Rightarrow P\left(r_{t} \mid \mathcal{F}_{t-1} \leq l\right) \leq \theta \Rightarrow P\left(\frac{r_{t} \mid \mathcal{F}_{t-1}-\mu_{t}}{\sigma_{t}} \leq \frac{l-\mu_{t}}{\sigma_{t}}\right) \leq \theta$.

Mas $\frac{r_{t} \mid \mathcal{F}_{t-1}-\mu_{t}}{\sigma_{t}} \sim N(0,1)$, assim, usando a inversa generalizada da função de distribuição acumulada da normal padrão $F_{N}$ e pela continuidade da distribuição normal, segue:

$$
\frac{l-\mu_{t}}{\sigma_{t}}=F_{N}^{-1}(\theta)=q_{N}(\theta)
$$

em que $q_{N}(\theta)$ é o $\theta$ - quantil da distribuição normal padrão. Assim:

$$
l=\mu_{t}+q_{N}(\theta) \sigma_{t} \text { e } \operatorname{logo} \operatorname{VaR}_{t}(\theta)=\mu_{t}+q_{N}(\theta) \sigma_{t} .
$$

Dado um horizonte $h$ :

$$
\operatorname{VaR}_{t+h}(\theta)=\mu_{t+h}+q_{N}(\theta) \sigma_{t+h} \Rightarrow \widehat{\operatorname{VaR}}_{t}(\theta)[h]=\hat{\mu}_{t}(h)+q_{N}(\theta) \hat{\sigma}_{t}(h) .
$$

em que $\hat{\mu}_{t}(h)$ e $\hat{\sigma}_{t}(h)$ são previsões da média e volatilidade da série de retornos $h$ passos à frente, obtidas através dos modelos ARMA-GARCH ajustados, com estruturas mostradas em (2.4), (2.5) e (2.6). 
Entretanto, assumir a normalidade dos erros, produz, em alguns casos, resultados que não são tão bons para o cálculo das medidas de risco por ser uma distribuição com caudas pesadas. Com o objetivo de obter melhores resultados, supõe-se a distribuição t - Student para os erros, como pode ser visto a seguir.

\subsection{VaR Usando a Distribuição t-Student}

Supondo

$$
\epsilon_{t} \sim t(0,1, v)
$$

em que $t(0,1, v)$ é a distribuição t-Student centrada usual, com $v$ graus de liberdade, cuja função densidade de probabilidade (f.d.p) é dada por:

$$
f(t \mid v)=\frac{\Gamma\left(\frac{v+1}{2}\right)}{\sqrt{v \pi} \Gamma\left(\frac{v}{2}\right)}\left(1+\frac{t^{2}}{v}\right)^{-\left(\frac{v+1}{2}\right)} .
$$

Considerando (2.3) e (2.9), $r_{t} \mid \mathcal{F}_{t-1}$ é definida como uma distribuição t-Student generalizada com parâmetros de posição $\mu_{t}$ e escala $\sigma_{t}^{2}$, ou seja, $r_{t} \mid \mathcal{F}_{t-1} \sim t\left(\mu_{t}, \sigma_{t}^{2}, v\right)$.

De fato

$$
\begin{aligned}
E\left\{r_{t} \mid \mathcal{F}_{t-1}\right\} & =\mu_{t} \mathrm{e} \\
\operatorname{Var}\left\{r_{t} \mid \mathcal{F}_{t-1}\right\} & =E\left\{r_{t}^{2} \mid \mathcal{F}_{t-1}\right\}-\left\{E\left(r_{t} \mid \mathcal{F}_{t-1}\right)\right\}^{2}= \\
& =E\left\{\mu_{t}^{2} \mid \mathcal{F}_{t-1}\right\}+E\left\{\left(\sigma_{t}^{2} \epsilon_{t}^{2}\right) \mid \mathcal{F}_{t-1}\right\}-\mu_{t}^{2}= \\
& =\mu_{t}^{2}+\sigma_{t}^{2}\left(\frac{v}{v-2}\right)-\mu_{t}^{2}=\sigma_{t}^{2}\left(\frac{v}{v-2}\right),
\end{aligned}
$$

em que $\sigma_{t}^{*}=\sqrt{\sigma_{t}^{2}\left(\frac{v}{v-2}\right)}=\sigma_{t} \sqrt{\frac{v}{v-2}}$ é o desvio padrão da distribuição t-Student de 
posição e escala.

Note que $\frac{r_{t} \mid \mathcal{F}_{t-1}-\mu_{t}}{\sigma_{t}} \sim t(0,1, v)$, definida em (2.9). Fazendo

$$
P\left(r_{t} \mid \mathcal{F}_{t-1} \leq l\right)=\theta \quad \Rightarrow \quad P\left(\frac{r_{t} \mid \mathcal{F}_{t-1}-\mu_{t}}{\sigma_{t}} \leq \frac{l-\mu_{t}}{\sigma_{t}}\right)=\theta .
$$

Usando a inversa generalizada da função de distribuição acumulada da t-Student usual com $v$ graus de liberdade $F_{t_{v}}$, tem -se:

$$
\frac{l-\mu_{t}}{\sigma_{t}}=F_{t_{v}}^{-1}(\theta)=q_{t_{v}}(\theta)
$$

onde $q_{t_{v}}(\theta)$ é o $\theta$ - quantil da distribuição dada em (2.9).

Mas

$$
\begin{aligned}
P\left(\frac{r_{t} \mid \mathcal{F}_{t-1}-\mu_{t}}{\sigma_{t}} \leq \frac{l-\mu_{t}}{\sigma_{t}}\right) & =\theta \Rightarrow \\
\Rightarrow P\left(\frac{r_{t} \mid \mathcal{F}_{t-1}-\mu_{t}}{\sigma_{t} \sqrt{\frac{v}{v-2}}} \leq \frac{l-\mu_{t}}{\sigma_{t} \sqrt{\frac{v}{v-2}}}\right) & =\theta,
\end{aligned}
$$

$\log 0$

$$
q_{t_{v}}^{*}(\theta)=\frac{l-\mu_{t}}{\sigma_{t} \sqrt{\frac{v}{v-2}}}=\frac{q_{t_{v}}(\theta)}{\sqrt{\frac{v}{v-2}}} .
$$

Daí, segue que:

$$
l=\mu_{t}+\sigma_{t} \sqrt{\frac{v}{v-2}} q_{t_{v}}^{*}(\theta) \Rightarrow \operatorname{VaR}_{t}(\theta)=\mu_{t}+\sigma_{t}^{*} q_{t_{v}}^{*}(\theta),
$$

em que $q_{t_{v}}^{*}(\theta)$ é o $\theta$ - quantil da distribuição t-student padronizada.

Assim como no ítem anterior, dado um horizonte $h$,

$$
V a R_{t+h}(\theta)=\mu_{t+h}+q_{t_{v}}^{*}(\theta) \sigma_{t+h}^{*} \Rightarrow \widehat{V a R}_{t}(\theta)[h]=\hat{\mu}_{t}(h)+q_{t_{v}}^{*}(\theta) \hat{\sigma}_{t}^{*}(h),
$$


em que $\hat{\mu}_{t}(h)$ e $\hat{\sigma}_{t}^{*}(h)$ são previsões $h$ passos à frente.

As duas distribuições atribuídas ao erro, comentadas anteriormente são simétricas, logo os retornos, dadas as informações passadas, também são simétricos. Com o objetivo de obter um cenário mais realista, a seguir, propõe-se uma nova distribuição, com novos parâmetros ligados a assimetria e curtose, capazes de representar melhor o comportamento das séries financeiras, nas quais o sinal dos retornos pode causar diferentes impactos nas medidas de risco.

\subsection{VaR Usando a Distribuição t-Student Genera- lizada Assimétrica}

Com relação a distribuições assimétricas, Fernándes e Steel (1998) apresentam um método geral para transformar uma distribuição simétrica em assimétrica através da indexação de um escalar $\psi \in(0,+\infty)$, denominado parâmetro de assimetria.

É interessante destacar que, além da distribuição a ser apresentada, existem outras densidades que também são conhecidas como t-assimétrica, porém este trabalho calcula medidas de risco utilizando a densidade assimétrica de acordo com Fernándes e Steel (1998).

Considerando uma v.a. $X$ com f.d.p. $f($.$) univariada, unimodal e simétrica em$ torno de zero, formalmente assumindo $f(x)=f(|x|)$, gera-se então uma nova classe de distribuições assimétricas, em que:

$$
f(x \mid \psi)=\frac{2 \psi}{\psi^{2}+1}\left\{f\left(\frac{x}{\psi}\right) I_{[0,+\infty)}(x)+f(x \psi) I_{(-\infty, 0)}(x)\right\}
$$

em que $\psi$ é denominado fator de escala.

A idéia é a introdução desse fator de escala, comportando-se de forma diferente conforme o sinal que a variável aleatória assume. 
Para a distribuição t-Student em (2.9), de acordo com (2.11),

$$
\begin{aligned}
f(z ; \nu, \psi)=C & \left\{\left(1+\frac{\left(\frac{z}{\psi}\right)^{2}}{\nu}\right)^{-\left(\nu+\frac{1}{2}\right)} I_{[0,+\infty)}(z)+\right. \\
& \left.+\left(1+\frac{(z \psi)^{2}}{\nu}\right)^{-\left(\nu+\frac{1}{2}\right)} I_{(-\infty, 0)}(z)\right\},
\end{aligned}
$$

em que $C=\left[\left(\psi+\psi^{-1}\right) 2^{-1}(\nu)^{\frac{1}{2}} B\left(\nu, \frac{1}{2}\right)\right]^{-1}, \psi$ e $\nu$ são parâmetros positivos, $B(.,$.$) é$ a função Beta.

Neste caso, $Z=\frac{T}{\sqrt{2}}$, onde $T \sim t(0,1, v)$ e $\frac{v}{2}=\nu .{ }^{1}$ Sendo assim, diz-se então, que $Z \sim t$ - Student Generalizada Assimétrica(tsga).

De fato, $Z$ seria um caso particular de:

$$
f(z ; d, \nu, \psi)=I_{(z<0)} C\left(1+\frac{(-z \psi)^{d}}{\nu}\right)^{-\left(\nu+\frac{1}{2}\right)}+I_{(z \geq 0)} C\left(1+\frac{\left(\frac{z}{\psi}\right)^{d}}{\nu}\right)^{-\left(\nu+\frac{1}{2}\right)},
$$

em que $d=2$.

Para um $d$ qualquer, o $m$-ésimo inteiro momento de $Z$, com $0 \leq m<\nu d$ é dado por

$$
E\left\{z^{m}\right\}=\frac{(-1)^{m} \psi^{-(m+1)}+\psi^{m+1}}{\psi^{-1}+\psi}\left[\frac{B\left(\frac{m+1}{d}, \nu-\frac{m}{d}\right)}{B\left(\frac{1}{d}, \nu\right)}\right] \nu^{\frac{m}{d}},
$$

e a função de distribuição acumulada de $Z$ é dada por

$$
F(z)= \begin{cases}\frac{1}{\left(1+\psi^{2}\right) B\left(\nu, \frac{1}{d}\right)}\left(\int_{0}^{\frac{\nu}{\nu+(-z \psi)^{d}}} t^{\nu-1}(1-t)^{\frac{1}{d}-1} d t\right), & \text { se } z \geq 0 \\ \frac{1}{\left(1+\psi^{-2}\right) B\left(\frac{1}{d}, \nu\right)}\left(\int_{0}^{\nu+\left(\frac{z}{\psi}\right)^{d}} t^{\frac{1}{d}-1}(1-t)^{\nu-1} d t\right)+\left(1+\psi^{2}\right), & \text { se } z<0\end{cases}
$$

\footnotetext{
${ }^{1}$ Para mais detalhes, ver Apêndice A.
} 
Considerando o modelo (2.3) com $\epsilon_{t} \sim \operatorname{tsga}\left(\nu_{t}, \psi_{t}\right)$ definida em $(2.12), r_{t} \mid \mathcal{F}_{t-1}$ segue uma t-Student Generalizada Assimétrica de posição $\mu_{t}$ e escala $\sigma_{t}$. Assim, como pode ser visto em Lambert e Laurent (2002),

$$
r_{t} \mid \mathcal{F}_{t-1} \sim \operatorname{tsga}\left(\mu_{t}, \sigma_{t}, \nu_{t}, \psi_{t}\right)
$$

com densidade

$$
\begin{aligned}
f\left(r_{t} \mid \mathcal{F}_{t-1} ; \nu_{t}, \psi_{t}\right) & =C\left\{\left(1+\frac{\left(\frac{r_{t} \mid \mathcal{F}_{t-1}-\mu_{t}}{\psi_{t} \sigma_{t}}\right)^{2}}{\nu_{t}}\right)^{-\left(\nu_{t}+\frac{1}{2}\right)} I_{[0,+\infty)}\left(r_{t} \mid \mathcal{F}_{t-1}-\mu_{t}\right)+\right. \\
& \left.+\left(1+\frac{\left[\left(\frac{r_{t} \mid \mathcal{F}_{t-1}-\mu_{t}}{\sigma_{t}}\right) \psi_{t}\right]^{2}}{\nu_{t}}\right)^{-\left(\nu_{t}+\frac{1}{2}\right)} I_{(-\infty, 0)}\left(r_{t} \mid \mathcal{F}_{t-1}-\mu_{t}\right)\right\} .
\end{aligned}
$$

De acordo com Lambert e Laurent (2002), quando o parâmetro de assimetria $\psi_{t}$ é diferente de $1, \mu_{t}$ e $\sigma_{t}^{2}$ não são mais a média e a variância, mas definidos como moda e parâmetro de dispersão, os quais são estimados através das estruturas de modelos ARMA-GARCH, considerando $\epsilon_{t}$ independentes e identicamente distribuídos.

O cálculo do VaR, assim como nos casos anteriores, é obtido a partir da definição de VaR.

Assim,

$$
\operatorname{VaR} R_{t+h}(\theta)=\mu_{t+h}+q_{t s g a}(\theta) \sigma_{t+h} \Rightarrow \widehat{\operatorname{VaR}}_{t}(\theta)[h]=\hat{\mu}_{t}(h)+q_{t s g a}(\theta) \hat{\sigma}_{t}(h),
$$

em que $q_{t s g a}(\theta)$ é o $\theta$ - quantil da distribuição t-Student assimétrica de posição e escala, definida em (2.14).

Como visto, para o cálculo do VaR, fazem-se as suposições necessárias à distribuição dos erros, e como já dito, esta é uma medida de risco muito utilizada, mas 
não é considerada uma Medida de Risco Coerente ${ }^{2}$ por não ser subaditiva, como pode ser visto em Acerbi e Tasche (2002).

Para mostrar como o VaR não satisfaz esse axioma, considere duas posições com perdas $L_{1}$ e $L_{2}$, independentes e identicamente distribuídas, com densidades tais que

$$
\begin{aligned}
P\left\{-2 \leq L_{i} \leq 0\right\} & =0,05 \\
P\left\{0 \leq L_{i} \leq 1\right\} & =0,90, \text { com } i=\{1,2\} .
\end{aligned}
$$

Pelo fato das únicas infomações serem as distribuições dadas em 2.15, calcula-se

$$
\operatorname{VaR}_{L_{i}}(0,1)=\inf \left\{l \in \mathbb{R}: P\left(L_{i} \geq l\right) \geq 0,9\right\} \Rightarrow \operatorname{VaR}_{L_{i}}(0,1)=0
$$

$\log \mathrm{O}$

$$
\operatorname{VaR}_{L_{1}}(0,1)+\operatorname{VaR}_{L_{2}}(0,1)=0
$$

Seja, $L=L_{1}+L_{2}$

$$
\operatorname{VaR}_{L}(0,1)=\operatorname{VaR}_{L_{1}+L_{2}}(0,1)=\inf \left\{l \in \mathbb{R}: P\left(L_{1}+L_{2} \geq l\right) \geq 0,9\right\},
$$

fazendo

$P\left(L_{1}+L_{2} \geq l\right)=P\left(L_{1}+L_{2} \geq l \mid L_{1} \geq l\right) P\left(L_{1} \geq l\right)+P\left(L_{1}+L_{2} \geq l \mid L_{1}<l\right) P\left(L_{1}<l\right)$.

Mas,

$P\left(L_{1}+L_{2} \geq l \mid L_{1} \geq l\right)=P\left(L_{2} \geq 0\right)$ e fazendo $P\left(L_{1}+L_{2} \geq l \mid L_{1}<l\right)=k$, em que $0 \leq k \leq 1$, $\log \mathrm{O}$

$$
\begin{aligned}
P\left(L_{1}+L_{2} \geq l\right) & =P\left(L_{2} \geq 0\right) P\left(L_{1} \geq l\right)+k P\left(L_{1}<l\right)= \\
& =0,95 P\left(L_{1} \geq l\right)+k\left(1-P\left(L_{1} \geq l\right)\right)= \\
& =P\left(L_{1} \geq l\right)(0,95-k)+k .
\end{aligned}
$$

\footnotetext{
${ }^{2}$ Ver Apêndice B.
} 
Deseja-se $P\left(L_{1}+L_{2} \geq l\right) \geq 0,9$, ou seja,

$$
P\left(L_{1} \geq l\right)(0,95-k)+k \geq 0,9 \Rightarrow P\left(L_{1} \geq l\right) \geq \frac{0,9-k}{0,95-k} .
$$

Analisando os valores de $P\left(L_{1} \geq l\right)$ de acordo com os valores de k:

- $P\left(L_{1} \geq l\right)$ atinge o seu máximo quando $k=0$;

- $P\left(L_{1} \geq l\right)$ atinge o seu mínimo quando $k=0,9$;

Assim, $0 \leq P\left(L_{1} \geq l\right) \leq 0,94$, e de acordo com (2.15),

$$
\begin{aligned}
\operatorname{VaR}_{L_{1}+L_{2}}(0,1) & =\inf \left\{l \in \mathbb{R}: 0 \leq P\left(L_{1} \geq l\right) \leq 0,94\right\} \Rightarrow \\
\Rightarrow \operatorname{VaR}_{L_{1}+L_{2}}(0,1) & >0
\end{aligned}
$$

Note que, de acordo com (2.16) e (2.17):

$$
\operatorname{VaR}_{L_{1}+L_{2}}(0,1)>\operatorname{VaR}_{L_{1}}(0,1)+\operatorname{VaR}_{L_{2}}(0,1)=0
$$

Ou seja, considerando

$$
\operatorname{VaR}_{L_{i}}(0,1)=\omega\left(L_{i}\right)
$$

a medida de risco,

$$
\omega\left(L_{1}+L_{2}\right)>\omega\left(L_{1}\right)+\omega\left(L_{2}\right) .
$$

Assim, o VaR se mostra uma medida de Risco não subaditiva, logo não é coerente.

É importante destacar também, que em outros casos, como por exemplo supondo erros com distribuição normal, a medida é subaditiva.

Assim, se

$$
\begin{aligned}
& L_{i} \stackrel{i i d}{\sim} N\left(\mu_{i}, \sigma_{i}^{2}\right), i \in(1,2) \\
& \operatorname{VaR}_{L_{i}}(\theta)=\mu_{i}+\sigma_{i} q_{N}(\theta) \Rightarrow \\
& \Rightarrow \operatorname{VaR}_{L_{1}}(\theta)+V a R_{L_{2}}(\theta)=\left(\mu_{1}+\mu_{2}\right)+\left(\sigma_{1}+\sigma_{2}\right) q_{N}(\theta)
\end{aligned}
$$


mas,

$$
L \sim N\left(\mu_{1}+\mu_{2}, \sigma_{1}^{2}+\sigma_{2}^{2}\right), \text { assim, } \operatorname{VaR}_{L}(\theta)=\left(\mu_{1}+\mu_{2}\right)+\sigma q_{N}(\theta) .
$$

Note que $\sigma^{2}=\sigma_{1}^{2}+\sigma_{2}^{2} \Rightarrow \sigma=\sqrt{\sigma_{1}^{2}+\sigma_{2}^{2}} \leq \sigma_{1}+\sigma_{2}$.

$$
\therefore \operatorname{VaR}_{L}(\theta) \leq \operatorname{VaR}_{L_{1}}(\theta)+\operatorname{VaR}_{L_{2}}(\theta) \text {. }
$$

Logo, para a distribuição normal, a medida é coerente, mas de forma geral, como no caso em que a distribuição não é simétrica em torno de zero, como no exemplo, a medida é não coerente. Além disso, o VaR com as estruturas apresentadas, fornece informação de um quantil, mas está ligado diretamente ao desvio padrão, podendo apresentar medidas de risco insatisfatórias dependendo do comportamento dos retornos, com perdas apresentando valores autocorrelacionados ao longo do tempo. 


\section{Capítulo 3}

\section{TVE - Teoria dos Valores Extremos}

\subsection{Conceitos}

O cálculo do VaR utilizando a Teoria dos Valores Extremos (TVE) pode ser feito por vários métodos, como por exemplo através das estatísticas de ordem. Neste caso, a série de retornos observada é dividida em $m$ blocos, obtendo-se depois os máximos de cada bloco, aos quais a TVE é aplicada, ou seja, ajusta-se uma distribuição Generalizada de Valores Extremos (GVE) a esses máximos, estima-se o quantil desta distribuição, a partir do qual é possível obter o VaR de uma posição ${ }^{1}$. Outras estatísticas também podem ser utilizadas, como por exemplo, considerar as $r$ maiores estatísticas de ordem em cada bloco.

Muitas vezes, considerando somente os máximos de cada bloco, há uma considerável perda de informação para a análise de valores extremos se outros dados nos extremos não são avaliados. Por isso, o método dos "excessos threshold"tem sido muito utilizado, onde todos os dados que excedem um limiar são avaliados, obtendo uma máxima informação possível dos dados.

Seja $X_{1}, X_{2}, \ldots, X_{n}$ uma sequência de v.as. independentes e identicamente distribuídas com função de distribuição $F($.$) e considere M_{n}=\max \left\{X_{1}, X_{2}, \ldots, X_{n}\right\}$,

\footnotetext{
${ }^{1}$ Para mais detalhes, ver Morettin(2008)
} 
$\log \mathrm{O}$

$$
\begin{aligned}
P\left\{M_{n} \leq x\right\} & =P\left\{\max \left(X_{1}, X_{2}, \ldots, X_{n}\right) \leq x\right\}= \\
& =P\left\{X_{1} \leq x, X_{2} \leq x, \ldots, X_{n} \leq x\right\}= \\
& =\prod_{i=1}^{n} P\left\{X_{i} \leq x\right\}=[F(x)]^{n} .
\end{aligned}
$$

Da TVE tem-se que, se existem sequências de constantes $\left\{a_{n}>0\right\}$ e $\left\{b_{n}\right\}$ tais que

$$
P\left\{\frac{M_{n}-b_{n}}{a_{n}} \leq x\right\} \longrightarrow G(x),
$$

para uma distribuição não degenerada $G$, quando $n \rightarrow \infty$, então $G$ pertence a uma das três famílias de distribuições de valores extremos, que podem ser escritas de forma única como sendo:

$$
G(x)=\exp \left\{-\left[1+\xi\left(\frac{x-\psi}{\beta}\right)\right]^{-1 / \xi}\right\},
$$

$\operatorname{para} \operatorname{algum} \psi, \beta>0$ e $\xi$.

Agora, considerando um limiar $u$ e a mesma sequência de v.a. $X_{i}$, e denotando um termo arbitrário de $X_{i}$ por $X$, pelo comportamento estocástico de eventos extremos, a distribuição de $X$ que excede um limiar $u$ é dada por:

$$
\begin{aligned}
P\{X-u>y \mid X>u\} & =\frac{P\{X>y+u, X>u\}}{P\{X>u\}}=\frac{P\{X>y+u\}}{P\{X>u\}} \\
& =\frac{1-F(y+u)}{1-F(u)}=1-F_{u}(y)
\end{aligned}
$$

em que $F_{u}(y)$ é a função de distribuição da v.a. $Y=\{X-u \mid X>u\}$. 
Assim, segundo (3.1) e (3.2), pode-se escrever:

$$
\begin{aligned}
F^{n}(x) & \approx \exp \left\{-\left[1+\xi\left(\frac{x-\psi}{\beta}\right)\right]^{-1 / \xi}\right\} \\
\Rightarrow n \log F(x) & \approx-\left[1+\xi\left(\frac{x-\psi}{\beta}\right)\right]^{-1 / \xi} \\
\Rightarrow \log F(x) & \approx-\frac{1}{n}\left[1+\xi\left(\frac{x-\psi}{\beta}\right)\right]^{-1 / \xi} .
\end{aligned}
$$

Para valores grandes de $x$, a expansão de Taylor indica que:

$$
\log F(x) \approx-\{1-F(x)\}
$$

Para valores altos de $u$, de acordo com (3.4):

$$
1-F(u) \approx \frac{1}{n}\left[1+\xi\left(\frac{u-\psi}{\beta}\right)\right]^{-1 / \xi} .
$$

Da mesma forma, para um $y>0$ :

$$
1-F(y+u) \approx \frac{1}{n}\left[1+\xi\left(\frac{y+u-\psi}{\beta}\right)\right]^{-1 / \xi}
$$

Logo, por (3.3):

$$
\begin{aligned}
P\{X>y+u \mid X>u\} & \approx \frac{n^{-1}\left[1+\xi\left(\frac{y+u-\psi}{\beta}\right)\right]^{-1 / \xi}}{n^{-1}\left[1+\xi\left(\frac{u-\psi}{\beta}\right)\right]^{-1 / \xi}}= \\
& =\left[\frac{1+\xi\left(\frac{u-\psi}{\beta}\right)}{1+\xi\left(\frac{u-\psi}{\beta}\right)}+\frac{\frac{\xi(y)}{\beta}}{1+\xi\left(\frac{u-\psi}{\beta}\right)}\right]^{-1 / \xi}= \\
& =\left[1+\frac{\frac{\xi(y)}{\beta}}{\frac{\beta+\xi(u-\psi)}{\beta}}\right]^{-1 / \xi}=\left[1+\frac{\xi(y)}{\tilde{\beta}}\right]^{-1 / \xi}
\end{aligned}
$$

em que $\tilde{\beta}=\beta+\xi(u-\psi)$. 
Assim, para um valor $u$ suficientemente grande, a função de distribuição dos valores da v.a. $X-u$ que excede o valor $y$, condicional em $X>u$, é aproximadamente:

$$
H(y)=1-\left[1+\frac{\xi(y)}{\beta}\right]^{-1 / \xi},
$$

definida em $\{y: y>0\}$ e $\left(1+\frac{\xi y}{\beta}\right)>0$.

As famílias de distribuições definidas em (3.5) são chamadas Distribuições Generalizadas de Pareto (DGP), ou seja, considerando um limiar $u$, e uma v.a. $X$, os excessos $X-u \mid X>u$ seguem uma DGP, como pode ser visto em Coles (2001).

Com o objetivo de estimar um quantil da distribuição dada em (3.5), tem-se que de acordo com (3.3)

$$
\begin{aligned}
F_{u}(y) & =1-\frac{1-F(y+u)}{1-F(u)}=\frac{F(y+u)-F(u)}{1-F(u)} \\
\Rightarrow F(y+u) & =F_{u}(y)[1-F(u)]+F(u),
\end{aligned}
$$

considerando $w=y+u$,

$$
F(w)=F_{u}(w-u)[1-F(u)]+F(u),
$$

em que $F_{u}(w-u)$ pode ser estimado por $H(w-u)$ e $F(u)$ pode ser estimado pela função de distribuição empírica $F_{n}(u)$.

O parâmetro $\xi$ estimado para $F_{u}(y)$ tem a mesma forma para $F_{u}(w-u)$, o que não ocorre $\operatorname{com} \beta$, pois ao considerar os valores $w=y+u$, existirá, para cada valor $u$ adotado um parâmetro $\beta_{u}$, que será estimado por máxima-verossimilhança.

Logo,

$$
\begin{aligned}
\hat{F}(w) & =H(w-u)\left[1-F_{n}(u)\right]+F_{n}(u) \\
\Rightarrow 1-\hat{F}(w) & =1-F_{n}(u)-H(w-u)\left[1-F_{n}(u)\right] \\
& =\left(1-F_{n}(u)\right)[1-H(w-u)] .
\end{aligned}
$$

Seja $1-F_{n}(u)=\overline{F_{n}(u)}, \log 0$ 


$$
1-\hat{F}(w)=\overline{F_{n}(u)}\left[1+\frac{\hat{\xi}(w-u)}{\hat{\beta}_{u}}\right]^{-1 / \hat{\xi}}
$$

Para $\hat{F}(w)=\theta$ e $\theta>1-F(u)$, pode-se inverter (3.6), ou seja:

$$
\begin{aligned}
(1-\theta)^{-\hat{\xi}} & =\left(\overline{F_{n}(u)}\right)^{-\hat{\xi}}\left[1+\frac{\hat{\xi}(q(\theta)-u)}{\hat{\beta}_{u}}\right] \Rightarrow \\
\Rightarrow\left(\frac{1-\theta}{\overline{F_{n}(u)}}\right)^{-\hat{\xi}}-1 & =\frac{\hat{\beta}_{u}}{\hat{\xi}}(q(\theta)-u) \Rightarrow \\
\Rightarrow \hat{q}(\theta) & =u+\frac{\hat{\beta}_{u}}{\hat{\xi}}\left[\left(\frac{1-\theta}{\overline{F_{n}(u)}}\right)^{-\hat{\xi}}-1\right] \Rightarrow \\
\Rightarrow \hat{q}(\theta) & =u+\frac{\hat{\beta}_{u}}{\hat{\xi}}\left[\left(\frac{n}{N_{u}}(1-\theta)\right)^{-\hat{\xi}}-1\right]
\end{aligned}
$$

em que $N_{u}$ é o número de excessos acima do limiar $u$ e $n$ o número total de realizações obtidas da distribuição $F($.$) .$

Assim, $\hat{q}(\theta)$ é a estimativa do $\theta$ - quantil da distribuição de $X$ que excede $u$.

Ao utilizar a TVE para estimar o VaR, o trabalho apresenta dois métodos, os quais denominam-se VaR(TVE1) e VaR(TVE2), respectivamente.

\section{$3.2 \quad \operatorname{VaR}(\mathrm{TVE} 1)$}

Este método, discutido por Zivot (2006), considera uma série de retornos $r_{t}$, e aos excessos dos retornos após a escolha de um limiar apropriado ajusta-se uma DGP, de acordo com o que foi visto anteriormente.

Da definição de VaR, e de acordo com (3.7), considerando a distribuição de uma v.a. que excede um threshold $u$, o VaR(TVE1), para uma posição vendida, pode ser 
calculado como:

$$
\widehat{V a R}_{t}(T V E 1)(\theta)=u+\frac{\hat{\beta}_{t, u}}{\hat{\xi}_{t}}\left[\left(\frac{1-\theta}{\overline{F_{n}(u)}}\right)^{-\hat{\xi}_{t}}-1\right],
$$

em que $\xi_{t}$ e $\beta_{t, u}$ são os parâmetros estimados considerando a série de log-retornos até $\mathrm{o}$ instante $t-1$.

Como pode ser visto, o cálculo do VaR está intimamente relacionado com a escolha do limiar $u$, assim, visando reponder a questão de qual seria o melhor limiar a ser adotado, faz-se um pequeno estudo de simulação. McNeil e Saladin (1997) apresentam um método que utiliza o seguinte procedimento:

1 - Através de uma distribuição $F($.$) com caudas pesadas, fixa-se a probabilidade$ $0<<\phi<\theta<1$ e $N_{u}>0$ o número observãções acima do limiar $u$;

2 - Calcule $u=q(\phi)$ usando (3.7). O threshold é obtido como sendo o $\phi$-quantil de $F($.$) ;$

3 - Calcule $q(\theta)$ o verdadeiro valor do quantil a ser estimado, em que

$$
q(\theta)=\inf \{w \in \mathbb{R}: F(w) \geq \theta\}
$$

4 - Obtenha $N_{u}$ pontos independentes de F acima do threshold $u$ pelo método da aceitação-rejeição ${ }^{2}$ e obtenha o número total de pontos n necessários;

5 - Estime $\xi$ e $\beta$, ajustando uma DGP para os $N_{u}$ excessos acima do threshold $u$, por máxima-verossimilhança;

6 - Calcule $\hat{q}(\theta)$ a estimativa quantílica da DGP dada em (3.7);

\footnotetext{
${ }^{2}$ Ver Apêndice C.
} 
7 - Repita K vezes os passos 4 a 6 e estime o viés e o erro quadrático médio (EQM) da estimativa quantílica, onde:

$$
\begin{gathered}
\widehat{V i e ́ s}(\hat{q}(\theta))=E[\hat{q}(\theta)-q(\theta)]=\frac{1}{K} \sum_{i=1}^{K} \hat{q}_{i}(\theta)-q(\theta), \\
\widehat{E Q M}(\hat{q}(\theta))=E\left[(\hat{q}(\theta)-q(\theta))^{2}\right]=\frac{1}{K} \sum_{i=1}^{K}\left[\hat{q}_{i}(\theta)-q(\theta)\right]^{2} .
\end{gathered}
$$

Com o procedimento, o limiar pode ser alterado através da variação dos valores de $\phi, \theta$ e $N_{u}$. Logo, o valor de $u$ mais adequado é aquele que apresenta os menores valores para a estimativa do viés e do EQM.

Além do método mencionado anteriormente, há uma outra forma, pela qual o valor do limiar pode ser deduzido.

Considere, novamente a distribuição dos excessos $X-u$ condicional em $X>u$, seguindo uma DGP, em que a função densidade de probabilidade (fdp) é dada pela derivada de $H(y)$, definida em (3.5), ou seja:

$$
h(y)=d H(y) \Rightarrow h(y)=\frac{1}{\beta_{u}}\left[1+\frac{\xi y}{\beta_{u}}\right]^{-(1+\xi) / \xi} .
$$

Logo, é possível calcular a média de $Y=\{X-u \mid X>u\}$, como sendo:

$$
E\{Y\}=E\{X-u \mid X>u\}=\int_{0}^{\infty} y h(y) d y=\int_{0}^{\infty} y \frac{1}{\beta_{u}}\left[1+\frac{\xi y}{\beta_{u}}\right]^{-(1+\xi) / \xi} d y .
$$

Fazendo

$$
w=1+\frac{\xi y}{\beta_{u}} \Rightarrow \frac{\beta_{u}}{\xi}(w-1)=y,
$$


tem-se:

$$
d y=\frac{\beta_{u}}{\xi} d w
$$

De acordo com (3.9), para $y \rightarrow \infty, w \rightarrow \infty$ e para $y=0$ tem-se $w=1$, o que implica que substituindo (3.9) e (3.10) em (3.8), obtém-se

$$
\begin{aligned}
E\{X-u \mid X>u\} & =\lim _{a \rightarrow \infty}\left[\int_{1}^{a} \frac{\beta_{u}}{\xi^{2}}(w-1) w^{-(1+\xi) / \xi}\right] \\
& =\left.\frac{\beta_{u}}{\xi^{2}} \lim _{a \rightarrow \infty}\left[\frac{\xi}{\xi-1} w^{(\xi-1) / \xi}+\xi w^{-1 / \xi}\right]\right|_{1} ^{a} \\
& =\frac{\beta_{u}}{\xi^{2}} \lim _{a \rightarrow \infty}\left[\frac{\xi}{\xi-1} a^{(\xi-1) / \xi}+\xi a^{-1 / \xi}-\left(\frac{\xi^{2}}{\xi-1}\right)\right] \\
& =\frac{\beta_{u}}{\xi^{2}} \lim _{a \rightarrow \infty}\left[\frac{\xi a+\xi(\xi-1)}{(\xi-1) a^{1 / \xi}}+\frac{\xi^{2}}{1-\xi}\right] .
\end{aligned}
$$

Aplicando a regra de L'Hospital

$$
E\{X-u \mid X>u\}=\frac{\beta_{u}}{1-\xi},
$$

em que a média de uma DGP pode ser calculada para valores de $\xi$, tais que $0<\xi<1$.

Considere a variável $Y-\delta \mid Y>\delta$, para um $\delta$ suficientemente grande, onde $\delta>u$ e $Y$ segue uma DGP com média finita para $0<\xi<1$.

De fato, faz-se, de acordo com (3.3),

$$
\begin{aligned}
1-F_{\delta}(y) & =\frac{1-F(y+\delta)}{1-F(\delta)}=\frac{1-[F(u+(y+\delta-u))]}{1-[F(u+(\delta-u))]} \cdot \frac{(1-F(u))}{(1-F(u))}= \\
& =\frac{1-[F(u+(y+\delta-u))]}{(1-F(u))} \cdot \frac{(1-F(u))}{1-[F(u+(\delta-u))]}= \\
& =\frac{1-F_{u}[y+(\delta-u)]}{1-F_{u}(\delta-u)}=1-F_{(\delta-u)}(y) .
\end{aligned}
$$

Sendo assim, ao considerar uma v.a. $Y$, seguindo uma DGP com um limiar $u$, ao considerar um novo limiar $\delta>u, Y-\delta \mid Y>\delta$ também segue uma DPG, em que, de acordo com Coles (2001), o parâmetro $\xi$ tem a mesma forma do que o da v.a. $Y$, 
porém o parâmetro de escala $\beta_{\delta}$ depende do limiar utilizado. Sendo assim, dado que o parâmetro de escala para $Y$ é $\beta_{u}$, para $Y-\delta \mid Y>\delta$, $\operatorname{com} \delta>u$ é

$$
\beta_{\delta}=\beta_{u}+\xi(\delta-u) .
$$

Logo, a média é dada por

$$
E\{Y-\delta \mid Y>\delta\}=\frac{\beta_{\delta}}{1-\xi}=\frac{\beta_{u}+\xi(\delta-u)}{1-\xi} .
$$

Considere agora um novo limiar $\delta_{1}$, tal que $\delta_{1}>\delta$.

Logo,

$$
\beta_{\delta_{1}}=\beta_{\delta}+\xi\left(\delta_{1}-\delta\right)
$$

e por (3.11),

$$
\begin{aligned}
& \beta_{\delta_{1}}=\beta_{u}+\xi\left(\delta_{1}-\delta\right)+\xi\left(\delta_{1}-\delta\right) \Rightarrow \\
& \beta_{\delta_{1}}=\beta_{u}+\xi\left(\delta_{1}-u\right),
\end{aligned}
$$

em que

$$
E\left\{Y-\delta_{1} \mid Y>\delta_{1}\right\}=\frac{\beta_{u}+\xi\left(\delta_{1}-u\right)}{1-\xi} .
$$

Assim, considerando um novo limiar, ao calcular a função, definida como função média dos excessos, pode-se observar que a média é uma função linear da escolha do limiar.

De acordo com essa idéia, surge um método gráfico, detalhado por Tsay (2005) e Zivot (2006), que ajuda na dedução de um valor apropriado para o limiar, apresentado a seguir.

Considere uma amostra aleatória $Y_{1}, Y_{2}, \ldots, Y_{m}$. Seja $E_{N_{u}}(u)$ a função média empírica dos excessos, em que

$$
E_{N_{u}}(u)=\frac{1}{N_{u}} \sum_{i=1}^{N_{u}}\left(Y_{(i)}-u\right),
$$

em que $Y_{(i)}\left(i=1, \ldots, N_{u}\right)$ são valores tais que $Y_{(i)}>u$. Logo o gráfico de $E_{N_{u}}(u)$ deve ser linear em $\mathrm{u}$, para um valor fixo de $\xi$, e o valor deduzido como o apropriado para $u$ será o que apresentar o menor valor para $E_{N_{u}}(u)$. 


\subsection{VaR(TVE2)}

Considere uma série de retornos $r_{t}$, sob o modelo $r_{t}=\mu_{t}+\sigma_{t} \epsilon_{t}$ explicado anteriormente. Para o cálculo do VaR(TVE2), inicialmente ajusta-se um modelo $\operatorname{ARMA}(\mathrm{p}, \mathrm{q})$ - GARCH(r,s) com $\epsilon_{t} \sim f($.$) e obtém-se os resíduos padronizados do modelo$

$$
z_{t}=\frac{r_{t}-\hat{\mu}_{t}}{\hat{\sigma}_{t}}
$$

Em seguida, escolhe-se um limiar apropriado, e aos resíduos que excedem o limiar é ajustada uma DGP, obtendo as estimativas dos parâmetros $\hat{\xi}$ e $\hat{\beta}_{u}$, e de acordo com (3.7), o $\theta-$ quantil $q_{D G P}(\theta)$.

Assim, pela definição de VaR para séries variando no tempo:

$$
\begin{aligned}
\operatorname{VaR}_{t+h}(T V E 2)(\theta) & =\mu_{t+h}+q_{D G P}(\theta) \sigma_{t+h} \Rightarrow \\
\widehat{V a R}_{t}(T V E 2)(\theta)[h] & =\hat{\mu}_{t}(h)+q_{D G P}(\theta) \hat{\sigma}_{t}(h),
\end{aligned}
$$

em que, novamente, $\hat{\mu}_{t}(h)$ e $\hat{\sigma}_{t}(h)$ são previsões $h$ passos a frente obtidas através do modelo $\operatorname{ARMA}(\mathrm{p}, \mathrm{q})$ - GARCH$(\mathrm{r}, \mathrm{s})$ ajustado.

Ao calcular as estimativas do VaR, muitos gerenciadores de medidas de risco esperam errar o menos possível. Mas como saber se as estimativas do VaR são adequadas?

Visando reponder esta questão, a seguir, será apresentado um teste para fim de verificação, visando saber qual método pode ser considerado adequado.

\subsection{Verificação dos Métodos para o VaR}

Existem órgãos reguladores para validar ou não se essas medidas realmente expressam bem o comportamento do mercado, pois o objetivo é analisar se um modelo é capaz de prever os riscos corretamente.

Uma maneira mais simples de verificar a adequação do método é verificar a precisão do mesmo, ou seja, registrar a taxa de erro, que é a proporção de vezes que o VaR é excedido pelos log-retornos. 
No caso do trabalho, considera-se que para cada instante de tempo $t=(1, \ldots, T)$, $r_{i}$ é independente de $r_{j}, i \neq j$, onde $i, j=(1, \ldots, T)$.

Primeiramente, formula-se, para posições compradas:

$$
\left\{\begin{array}{l}
H_{0}: P\left\{r_{t}<\operatorname{VaR}(\theta)\right\}=\theta, \forall t, \\
H_{1}: P\left\{r_{t}<\operatorname{VaR} R_{t}(\theta)\right\}>\theta .
\end{array}\right.
$$

Sendo assim, constrói-se uma sequência de funções indicadoras, em que:

$$
I_{t}=1_{\left\{r_{t}<\operatorname{VaR}(\theta)\right\}} \sim \operatorname{Ber}(\theta) .
$$

Logo, considerando (3.12) e assumindo independência, para a série de retornos, tem-se a estatística

$$
\sum_{t \in T} I_{t} \sim \operatorname{Binomial}(\#(T), \theta),
$$

em que:

$$
\left\{\begin{array}{l}
H_{0}: \text { O método estima corretamente o quantil condicional; } \\
H_{1}: \text { O método tem um erro sistemático, fornecendo várias violações. }
\end{array}\right.
$$

Logo, se a proporção de valores que ultrapassam os valores estimados para o VaR estiver próxima de $\theta$, a um nível de significância estabelecido, há indícios de que o método é adequado.

É possível, também, construir intervalos de confiança para o número aceitável de violações, dado que estes números seguem uma distribuição Binomial.

No trabalho, esse método de verificação é utilizado para as estimativas do VaR obtidas através dos métodos que utlilizam abordagem econométrica e TVE, dados neste Capítulo e no Capítulo 2. 


\section{Capítulo 4}

\section{CAViaR - Valor em Risco Autorregressivo Condicional}

\subsection{Conceitos}

Analisando todas as fragilidades do VaR, Engle e Manganelli (2004) propuseram uma nova metodologia para o cálculo desta medida de risco, os modelos CAViaR(Valor em Risco Autoregressivo Condicional), que ao invés de modelar toda a distribuição dos retornos, modela diretamente seus quantis.

Os retornos, em muitos casos são autocorrelacionados, logo o VaR que é ligado ao desvio padrão pode apresentar comportamento semelhante. Assim com uma estrutura similar aos modelos GARCH, os modelos CAViaR, através de um termo autorregressivo, incorporam as autocorrelações do VaR ao modelo.

Segundo Kuester, Mittnik e Paolella (2006), a idéia básica é modelar o $\theta$-quantil condicional $Q_{\theta}\left(r_{t} \mid \boldsymbol{x}_{t}\right)=\operatorname{Va} R_{t}(\theta)$ como uma função $g\left(\right.$.) das informações $\boldsymbol{x}_{t} \in \mathcal{F}_{t-1}$ (informações até o instante $(t-1))$, e do vetor de parâmetros $\boldsymbol{\beta}$, ou seja, considerando posição vendida,

$$
\operatorname{VaR}(\theta) \equiv g_{\theta}\left(\boldsymbol{x}_{t}, \boldsymbol{\beta}_{\theta}\right)
$$


em que $r_{t} \operatorname{com}\{t: 1, \ldots, T\}$ são os retornos, $\theta$ a probabilidade associada ao VaR, $\boldsymbol{\beta}$ o vetor de parâmetros dependente de $\theta$.

Os modelos CAViaR possuem especificações distintas, as quais são utilizadas de acordo com o comportamento dos retornos. Estes modelos não fazem suposições sobre a distribuição dos retornos.

Uma forma mais geral da especificação do modelo é:

$$
\operatorname{VaR}_{t}(\theta)=\beta_{0}+\sum_{i=1}^{p} \beta_{i} \operatorname{Va} R_{t-i}(\theta)+g\left(\beta_{p+1}, \ldots, \beta_{p+q}, \mathcal{F}_{t-1}\right) .
$$

Por simplicidade, assim como nos modelos GARCH a especificação mais utilizada é a de ordem 1:

$$
\operatorname{VaR}_{t}(\theta)=\beta_{0}+\beta_{1} \operatorname{VaR}_{t-1}(\theta)+g\left(\beta_{2}, r_{t-1}, \operatorname{VaR}_{t-1}(\theta)\right),
$$

em que $g(., .,$.$) é a função de ligação entre V a R_{t-1}(\theta)$ e $r_{t-1}$, que mede o quanto o $V a R$ pode variar no tempo baseado em novas informações dos log-retornos, como as curvas de impacto dos modelos GARCH, com parâmetros apresentando valores entre $(-1,1)$.

A seguir são apresentadas algumas formulações específicas:

\subsection{Especificações dos Modelos CAViaR}

\subsubsection{Valor Absoluto Simétrico:}

$$
\operatorname{VaR}_{t}(\theta)=\beta_{1}+\beta_{2} \operatorname{VaR}_{t-1}(\theta)+\beta_{3}\left|r_{t-1}\right| .
$$

Nesta especificação é possível ver que $g(.,$.$) é uma função modular, mostrando$ como o VaR responde simetricamente aos retornos passados, ou seja, observações de mesma magnitude e sinais diferentes são tratadas igualmente. 


\subsubsection{Inclinação Assimétrica:}

$$
\operatorname{VaR}_{t}(\theta)=\beta_{1}+\beta_{2} \operatorname{VaR} R_{t-1}(\theta)+\beta_{3} r_{t-1} I\left(r_{t-1}>0\right)+\beta_{4} r_{t-1} I\left(r_{t-1}<0\right),
$$

em que $I($.$) representa uma função indicadora.$

Neste caso, $g(.,$.$) considera os retornos positivos e negativos separadamente, mos-$ trando que o VaR não responde simetricamente aos dados, a não ser quando $\beta_{3}=\beta_{4}$.

\subsection{3 $\operatorname{GARCH}(1,1)$ Indireto:}

$$
\operatorname{VaR}_{t}(\theta)=\left(\beta_{1}+\beta_{2} V a R_{t-1}^{2}(\theta)+\beta_{3} r_{t-1}^{2}\right)^{\frac{1}{2}}
$$

Essa especificação pode ser usada, segundo Engle e Manganelli (2004) se os dados seguem realmente um processo GARCH com erros independentes e identicamente distribuídos (iid) gerados por um modelo de posição e escala.

\subsubsection{Adaptativo:}

$$
\operatorname{VaR}_{t}(\theta)=\operatorname{VaR}_{t-1}(\theta)+\beta_{1}\left\{\left[1+\exp \left[k\left(r_{t-1}-V a R_{t-1}(\theta)\right)\right]\right]^{-1}-\theta\right\} .
$$

De acordo com Engle e Manganelli (2004), nesta especificação $k$ é um número finito positivo. Quando $k \rightarrow \infty$, o último termo converge quase certamente para $\beta_{1}\left[I\left(r_{t-1} \leq \operatorname{VaR}_{t-1}(\theta)\right)-\theta\right]$. A principal idéia é que quando o VaR é excedido, ele pode ser aumentado, mas quando isso não ocorre, ele pode ser diminuído suavemente, ou seja, se o VaR é excedido, quando $k \rightarrow \infty$, acrescenta-se $\beta_{1}(1-\theta)$ ao valor do VaR, caso contrário, o valor a ser diminuído é $-\theta \beta_{1}$.

Esta estratégia tende a diminuir a probabilidade da sequência de retornos $\left\{r_{t-1} \leq V_{a} R_{t-1}(\theta)\right\}$, e fará isso também para aqueles valores que possuem comportamento contrário, diminuindo a possibilidade de ter uma série autocorrelacionada de retornos que excedam ou não o VaR. 
34CAPÍTULO 4. CAVIAR - VALOR EM RISCO AUTORREGRESSIVO CONDICIONAL

\subsubsection{Adaptativo Simétrico Proporcional:}

$$
\begin{array}{r}
\operatorname{VaR}_{t}(\theta)=\operatorname{VaR} R_{t-1}(\theta)+\beta_{1} \max \left\{0,\left|r_{t-1}\right|-V a R_{t-1}(\theta)\right\}- \\
-\beta_{2} \min \left\{0,\left|r_{t-1}\right|-V a R_{t-1}(\theta)\right\}
\end{array}
$$

\subsubsection{Valor Absoluto Assimétrico:}

$$
\operatorname{VaR}_{t}(\theta)=\beta_{1}+\beta_{2} \operatorname{VaR} R_{t-1}(\theta)+\beta_{3}\left|r_{t-1}-\beta_{4}\right|
$$

E finalmente, o trabalho apresenta uma especificação mais recente, detalhada a seguir:

\subsection{7 $\operatorname{AR}(1)-\operatorname{GARCH}(1,1)$ indireto:}

Pensando em um modelo que possa capturar a autocorrelação da média condicional da série de retornos, Kuester, Mittnik e Paolella (2006) estenderam a estrutura do modelo GARCH Indireto dado em (4.3). Primeiramente eles consideraram um modelo de posição e escala, no qual a média e a volatilidade seguem um modelo ARMA-GARCH.

Por simplicidade, seja o modelo $\operatorname{AR}(1)-\operatorname{GARCH}(1,1)$, em que

$$
\begin{aligned}
r_{t} & =\phi_{0}+\phi_{1} r_{t-1}+a_{t} \\
a_{t} & =\sigma_{t} \epsilon_{t} \\
\sigma_{t}^{2} & =c_{0}+c_{1} a_{t-1}^{2}+d_{1} \sigma_{t-1}^{2} .
\end{aligned}
$$

O valor do $\operatorname{VaR}_{t}(\theta)$, como visto anteriormente é obtido a partir do modelo $r_{t}=\mu_{t}+\sigma_{t} \epsilon_{t}$, supondo $\epsilon_{t} \stackrel{i i d}{\sim} f($.), ou seja, para uma posição vendida,

$$
\operatorname{VaR}_{t}(\theta)=\mu_{t}+\sigma_{t} q_{f}(\theta)
$$


em que $q_{f}(\theta)$ é o $\theta-q u a n t i l$ da distribuição de $\epsilon_{t}$. Assim,

$$
\sigma_{t}=\frac{V a R_{t}(\theta)-\mu_{t}}{q_{f}(\theta)} \Rightarrow \sigma_{t}^{2}=\left(\frac{V a R_{t}(\theta)-\mu_{t}}{q_{f}(\theta)}\right)^{2} .
$$

Substituindo (4.8) na estrutura do modelo GARCH dada em (4.7):

$$
\begin{aligned}
& \left(\frac{V a R_{t}(\theta)-\mu_{t}}{q_{f}(\theta)}\right)^{2}=c_{0}+c_{1} a_{t-1}^{2}+d_{1}\left(\frac{V a R_{t-1}(\theta)-\mu_{t-1}}{q_{f}(\theta)}\right)^{2} \Rightarrow \\
& \Rightarrow \frac{V a R_{t}(\theta)-\mu_{t}}{q_{f}(\theta)}=\left[c_{0}+c_{1} a_{t-1}^{2}+d_{1}\left(\frac{V a R_{t-1}(\theta)-\mu_{t-1}}{q_{f}(\theta)}\right)^{2}\right]^{\frac{1}{2}},
\end{aligned}
$$

e utilizando um modelo $\operatorname{AR}(1)$, com $\phi_{0}=0$,

$$
r_{t}=\phi_{1} r_{t-1}+a_{t} \Rightarrow a_{t}=r_{t}-\phi_{1} r_{t-1} \Rightarrow a_{t-1}=r_{t-1}-\phi_{1} r_{t-2},
$$

e substituindo em (4.9), tem-se:

$$
\begin{gathered}
\operatorname{VaR}_{t}(\theta)-\mu_{t}=q_{f}(\theta)\left[c_{0}+c_{1}\left(r_{t-1}-\phi_{1} r_{t-2}\right)^{2}+d_{1}\left(\frac{V a R_{t-1}(\theta)-\mu_{t-1}}{q_{f}(\theta)}\right)^{2}\right]^{\frac{1}{2}} \Rightarrow \\
V a R_{t}(\theta)=\mu_{t}+\left[\left(q_{f}(\theta)\right)^{2} c_{0}+\left(q_{f}(\theta)\right)^{2} c_{1}\left(r_{t-1}-\phi_{1} r_{t-2}\right)^{2}+d_{1}\left(V a R_{t-1}(\theta)-\mu_{t-1}\right)^{2}\right]^{\frac{1}{2}} .
\end{gathered}
$$

De acordo com (4.10):

$$
\begin{aligned}
E\left\{r_{t} \mid \mathcal{F}_{t-1}\right\} & =E\left\{\left(\phi_{1} r_{t-1}+a_{t}\right) \mid \mathcal{F}_{t-1}\right\} \\
\Rightarrow \mu_{t} & =\phi_{1} r_{t-1},
\end{aligned}
$$

logo, desenvolvendo a equação e reparametrizando, tem-se o modelo AR(1)-

\section{$\operatorname{GARCH}(1,1)$ Indireto:}

$$
\operatorname{VaR}_{t}(\theta)=\phi_{1} r_{t-1}+\left[\beta_{1}+\beta_{2}\left(\operatorname{VaR}_{t-1}(\theta)-\phi_{1} r_{t-2}\right)^{2}+\beta_{3}\left(r_{t-1}-\phi_{1} r_{t-2}\right)^{2}\right]^{\frac{1}{2}},
$$

em que $\beta_{1}=\left(q_{f}(\theta)\right)^{2} c_{0}, \beta_{2}=d_{1}$ e $\beta_{3}=\left(q_{f}(\theta)\right)^{2} c_{1}$.

Os parâmetros dos modelos CAViaR são estimados por Regressão Quantílica $(R Q)$, uma técnica mais robusta a valores atípicos, desenvolvida por cálculos de otimização. 


\subsection{Estimação do Modelo}

\subsubsection{Regressão Quantílica}

Introduzida por Koenker e Bassett (1978), a técnica de RQ permite caracterizar toda a distribuição condicional de uma variável resposta a partir de um conjunto de regressores.

Seja a v.a. $Y$ com função de distribuição $F(y)$, onde para algum $0<\theta<1$,

$$
F^{-1}(\theta)=\inf \{y \in \mathbb{R}: F(y) \geq \theta\}
$$

é chamado $\theta$-ésimo quantil de $Y$.

Agora considere um simples problema de teoria da decisão com a função de perda convexa descrita como a seguinte função linear

$$
\rho_{\theta}(u)=\left\{\begin{array}{l}
u(\theta-1), \text { se } u<0 \\
u \theta, \text { se } u>0
\end{array},\right.
$$

em que $u=(Y-b)$ é o desvio, $b \in \mathbb{R}$.

Fixando um valor $\theta$, é possível calcular a média da função de perda como sendo

$$
E\left\{\rho_{\theta}(u)\right\}=E\left\{\rho_{\theta}(Y-b)\right\}=(\theta-1) \int_{-\infty}^{b}(y-b) d F(y)+\theta \int_{b}^{+\infty}(y-b) d F(y) .
$$

Com o objetivo de encontrar o menor desvio, calcula-se o valor de $b$ que minimiza a média da função de perda, dada em (4.13), ou seja, basta diferenciar $E\left\{\rho_{\theta}(Y-b)\right\}$ com relação a $b$ e igualar a zero, assim:

$$
(1-\theta) \int_{-\infty}^{b} d F(y)-\theta \int_{b}^{+\infty} d F(y)=0 \Rightarrow F(b)-\theta=0 \Rightarrow F(b)=\theta .
$$

Desde que $F$ seja monótona, algum elemento de $\{y \in \mathbb{R}: F(y)=\theta\}$ minimiza a perda esperada. Quando a solução é única, 


$$
b=F^{-1}(\theta),
$$

caso contrário, tem-se um intervalo para $b$, no qual o menor elemento deve ser escolhido.

Note que por (4.12) e (4.14), a divisão da amostra em quantis pode ser feita através da minimização da função perda esperada com respeito à variável aleatória $Y$, ou seja, basta obter o valor de $b$ que minimiza

$$
C Q=\min _{b \in \mathbb{R}}\left[E\left\{\rho_{\theta}(Y-b)\right\}\right],
$$

em que a função $C Q$ é chamada de Critério Quantílico, onde $b=Q(\theta)$ é o $\theta$-quantil da v.a. Y.

Para o cálculo do quantil amostral, através da função de perda para o caso discreto, considere $\left\{Y_{1}, Y_{2}, \ldots, Y_{T}\right\}$ uma amostra aleatória da v.a. $Y$ e $F_{n}$ a função de distribuição empírica:

$$
F_{n}(y)=n^{-1} \sum_{i=1}^{n} I_{\left(Y_{i} \leq y\right)}
$$

em que $i \in\{1, \ldots, T\}$ e $n \in\{1, \ldots, T\}$, com $i \leq n$.

Escolhendo $b$ que minimiza a perda esperada, considerando $F_{n}($.$) dada em (4.15),$ faz-se

$$
C Q=\min _{b \in \mathbb{R}}\left[n^{-1} \sum_{i=1}^{n} \rho_{\theta}\left(Y_{i}-b\right)\right],
$$

e considerando um modelo linear

$$
Y_{i}=\boldsymbol{x}_{i}^{\prime} \boldsymbol{\beta}+u_{i} \Rightarrow u_{i}=Y_{i}-\boldsymbol{x}_{i}^{\prime} \boldsymbol{\beta},
$$

em que $\boldsymbol{x}_{i}^{\prime} \boldsymbol{\beta}$ são os regressores, de acordo com (4.16), tem-se que o vetor $\hat{\boldsymbol{\beta}}_{\boldsymbol{\theta}}$ é o vetor $\boldsymbol{\beta}$, tal que

$$
C Q=\min _{\boldsymbol{\beta}}\left[n^{-1} \sum_{i=1}^{n} \rho_{\theta}\left(Y_{i}-\boldsymbol{x}_{i}^{\prime} \boldsymbol{\beta}\right)\right]
$$


Assim,

$$
C Q=\min _{\boldsymbol{\beta}}\left(\frac{1}{n}\left\{\sum_{i: Y_{i} \geq \boldsymbol{x}_{i}^{\prime} \boldsymbol{\beta}} \theta\left|Y_{i}-\boldsymbol{x}_{i}^{\prime} \boldsymbol{\beta}\right|+\sum_{i: Y_{i}<\boldsymbol{x}_{i}^{\prime} \boldsymbol{\beta}}(1-\theta)\left|Y_{i}-\boldsymbol{x}_{i}^{\prime} \boldsymbol{\beta}\right|\right\}\right)
$$

resulta no cálculo da estimativa do vetor $\boldsymbol{\beta}_{\theta}$ de parâmetros, ou seja, $Q(\theta)$ é obtido a partir do vetor $\hat{\boldsymbol{\beta}}_{\theta}$ que minimiza a função perda esperada.

Para estender o conceito de Regressão Quantílica a uma série de retornos financeiros, existem algumas suposições básicas a serem feitas para que o cálculo do VaR, de acordo com as especificações citadas anteriormente, seja possível.

A suposição chave do modelo linear é que

$$
r_{t}=\boldsymbol{x}_{t}^{\prime} \boldsymbol{\beta}_{\boldsymbol{\theta}}+u_{t, \theta} \text { e } Q_{\theta}\left(u_{t, \theta} \mid \boldsymbol{x}_{\boldsymbol{t}}\right)=0
$$

em que $r_{t}$ são os retornos, $u_{t, \theta}$ é a parte aleatória, $\boldsymbol{x}_{\boldsymbol{t}}$ o vetor de regressores, com ordem $(k \times 1)$ para um $t$ fixo, $\boldsymbol{\beta}_{\boldsymbol{\theta}}$ o vetor de $k$ parâmetros a serem ajustados e $Q_{\theta}\left(u_{t, \theta} \mid \boldsymbol{x}_{\boldsymbol{t}}\right)$ o $\theta-q u a n t i l$ de $u_{t, \theta}$ condicional em $\boldsymbol{x}_{\boldsymbol{t}}$.

De fato, tomando $Q_{\theta}\left(u_{t, \theta} \mid \boldsymbol{x}_{\boldsymbol{t}}\right)=0$, o modelo supõe que os erros tenham valores próximos de zero, independentemente do quantil adotado, assim possibilitando que os quantis condicionais dos retornos, sejam relativamente iguais a $\boldsymbol{x}_{t}^{\prime} \boldsymbol{\beta}_{\boldsymbol{\theta}}$, ou seja,

$$
Q_{\theta}\left(r_{t} \mid \boldsymbol{x}_{\boldsymbol{t}}\right)=\boldsymbol{x}_{t}^{\prime} \boldsymbol{\beta}_{\boldsymbol{\theta}}
$$

logo, o $\theta$-quantil de $r_{t}$ dadas as informações até o instante $t$, é a parte determinística do modelo, ou seja, o conjunto de informações disponíveis até o período $t$.

Portanto, de (4.17)

$$
C Q=\min _{\boldsymbol{\beta}}\left(\frac{1}{T}\left\{\sum_{t: r_{t} \geq V a R_{t}(\theta)} \theta\left|r_{t}-V a R_{t}(\theta)\right|+\sum_{t: r_{t}<V a R_{t}(\theta)}(1-\theta)\left|r_{t}-\operatorname{VaR}_{t}(\theta)\right|\right\}\right)
$$


resulta na estimativa $\hat{\boldsymbol{\beta}}_{\boldsymbol{\theta}}$ do vetor $\boldsymbol{\beta}$ de parâmetros, necessária para o cálculo do $\operatorname{VaR}_{t}(\theta)$, que pode assumir as especificações mostradas na seção 2.1 .

Por exemplo, considerando a especificação $\operatorname{GARCH}(\mathbf{1}, \mathbf{1})$ Indireto, dada em (4.3), calculando o vetor $\hat{\boldsymbol{\beta}_{\boldsymbol{\theta}}}$, pelo CQ tem-se,

$$
\begin{array}{r}
C Q=\min _{\boldsymbol{\beta}}\left[\frac { 1 } { T } \left\{\sum_{t}\left(I_{r_{t}<\left(\beta_{0}+\beta_{1} V a R_{t-1}^{2}(\theta)+\beta_{2} r_{t-1}^{2}\right)^{\frac{1}{2}}}-\theta\right)\right.\right. \\
\left.\left.\left|r_{t}-\left(\beta_{0}+\beta_{1} V a R_{t-1}^{2}(\theta)+\beta_{2} r_{t-1}^{2}\right)^{\frac{1}{2}}\right|\right\}\right],
\end{array}
$$

e $\hat{\boldsymbol{\beta}}_{\theta}=\left\{\hat{\beta}_{0}, \hat{\beta}_{1}, \hat{\beta}_{2}\right\}^{T}$.

A função $C Q$ serve como uma alternativa a mais para a seleção e adequação de modelos, pois para cada especificação, considerando os parâmetros estimados, desejase o que resulta no menor valor de $C Q$.

Considerados como um caso particular de estimadores de mínimos absolutos, os estimadores por regressão quantílica possuem características que os definem como sendo mais robustos que os de mínimos quadrados por serem menos sensíveis ao comportamento das caudas das distribuições dos dados.

Segundo Engle e Manganelli (2004), os estimadores obtidos por regressão quantílica são consistentes e assintoticamente normais, ou seja, sob certas condições e utilizando como base o trabalho de Weiss (1991), demonstram que

$$
\hat{\boldsymbol{\beta}}_{\theta} \stackrel{p}{\longrightarrow} \boldsymbol{\beta}_{\theta}, T \longrightarrow \infty,
$$

em que

$$
C Q=\min _{\boldsymbol{\beta}}\left(\frac{1}{T}\left\{\sum_{t: r_{t} \geq \operatorname{VaR} R_{t}(\theta)} \theta\left|r_{t}-\operatorname{VaR}_{t}(\theta)\right|+\sum_{t: r_{t}<\operatorname{VaR} R_{t}(\theta)}(1-\theta)\left|r_{t}-\operatorname{VaR}_{t}(\theta)\right|\right\}\right) .
$$

Sob certas suposições, foi possível provar que

$$
\sqrt{T} \boldsymbol{A}_{T}^{-1 / 2} \boldsymbol{D}_{T}\left(\hat{\boldsymbol{\beta}}_{\theta}-\beta_{\theta}\right) \stackrel{d}{\longrightarrow} N(\mathbf{0}, \boldsymbol{I}),
$$


em que

$$
\begin{aligned}
\boldsymbol{A}_{T} & \equiv E\left[T^{-1} \theta(1-\theta) \sum_{t=1}^{T} \nabla^{\prime} V a R_{t}(\theta) \nabla V a R_{t}(\theta)\right], \\
\boldsymbol{D}_{T} & \equiv E\left[T^{-1} \sum_{t=1}^{T} h_{t}\left(0 \mid \mathcal{F}_{t}\right) \nabla^{\prime} \operatorname{VaR}_{t}(\theta) \nabla V a R_{t}(\theta)\right],
\end{aligned}
$$

onde $\nabla \operatorname{Va} R_{t}(\theta)$ é gradiente de $\operatorname{Va} R_{t}(\theta)$ e $h_{t}\left(0 \mid \mathcal{F}_{t}\right)$ a densidade condicional dos erros calculada em zero.

As matrizes $\boldsymbol{A}_{T}$ e $\boldsymbol{D}_{T}$ podem ser estimadas por

$$
\begin{aligned}
\hat{\boldsymbol{A}}_{T} & =T^{-1} \theta(1-\theta) \nabla^{\prime} \widehat{\operatorname{VaR}}_{t}(\theta) \nabla \widehat{V a R}_{t}(\theta), \\
\hat{\boldsymbol{D}}_{T} & =\left(2 T \hat{c}_{T}\right) \sum_{t=1}^{T} I\left(\left|r_{t}-\widehat{\operatorname{VaR}}_{t}(\theta)\right|<\hat{c}_{T}\right) \nabla^{\prime} \widehat{\operatorname{VaR}}_{t}(\theta) \nabla \widehat{V a R}_{t}(\theta),
\end{aligned}
$$

em que

$$
\hat{c}_{T}=\frac{1}{T} \sum_{t=1}^{T} \frac{\nabla \widehat{V a R}_{t}(\theta) \nabla^{\prime} \widehat{\operatorname{VaR}}_{t}(\theta)}{\widehat{\operatorname{VaR}}_{t}(\theta)} .
$$

\subsection{Teste de Ajuste dos Modelos}

Considerando uma série de retornos $r_{t}$, para uma posição comprada, para determinar se o modelo está corretamente especificado, tem-se o teste:

$$
\left\{\begin{array}{l}
H_{0}: P\left\{r_{t}<\operatorname{VaR}_{t}(\theta)\right\}=\theta, \forall t, \\
H_{1}: P\left\{r_{t}<\operatorname{VaR}_{t}(\theta)\right\} \neq \theta .
\end{array}\right.
$$

É possível obter, também uma sequência de funções indicadoras, tal que

$$
I\left(r_{t}<\operatorname{VaR}_{t}(\theta)\right)_{t=1}^{T}
$$

Assim, uma forma de testar se o modelo é válido, é verificar se a sequência de funções indicadoras é i.i.d.. Logo, uma propriedade que qualquer estimativa de $V a R$ 
deve satisfazer é se comportar como um filtro, capaz de transformar uma série autocorrelacionada e heteroscedástica em uma série independente de funções indicadoras.

Esta é uma condição necessária, mas não suficiente para avaliar o comportamento e desempenho do modelo quantílico, uma vez que esta não considera e nem incorpora a influência de outras variáveis ao modelo.

Sendo assim, Engle e Manganelli (2004) propuseram um novo teste que pode ser estendido para incorporar essas variáveis ao modelo.

Seja

$$
\operatorname{Hit}_{t}\left(\beta_{\theta}\right)=I\left(r_{t}<\operatorname{VaR}(\theta)\right)-\theta
$$

uma função que assume os valores $(1-\theta)$ quando $r_{t}$ é menor que o $\theta-$ quantil e o valor $-\theta$ caso contrário.

Considerando $f_{t-1} \in \mathcal{F}_{t-1}$,

$$
E\left\{\left(f_{t-1} H i t_{t}\right) \mid f_{t-1}\right\}=f_{t-1} E\left\{H i t_{t} \mid f_{t-1}\right\},
$$

o que mostra que $H_{i t} t_{t}$ é não correlacionada com qualquer subconjunto pertencente ao conjunto de informações passadas $\mathcal{F}_{t-1}$, ou seja, não há correlação entre $H i t_{t} \mathrm{e}$ qualquer $H i t_{t-l}$, para todo $l$.

Segundo os autores, uma maneira natural de fazer o teste é verificar se a estatística do teste

$$
\frac{1}{T^{\frac{1}{2}}} \boldsymbol{X}^{\prime}(\hat{\boldsymbol{\beta}}) \boldsymbol{H} \boldsymbol{i t}(\hat{\boldsymbol{\beta}})
$$

é significativamente diferente de zero, onde $\boldsymbol{x}_{t}(\hat{\boldsymbol{\beta}}), t=1, \ldots, T$ é um k-vetor, sendo uma linha de $\boldsymbol{X}^{\prime}(\hat{\boldsymbol{\beta}})$, possivelmente dependendo de $\hat{\boldsymbol{\beta}}$, mensurável em $\mathcal{F}_{t-1}$, e

$$
\boldsymbol{H i t}(\hat{\boldsymbol{\beta}}) \equiv\left[\operatorname{Hit}_{1}(\hat{\boldsymbol{\beta}}), H i t_{2}(\hat{\boldsymbol{\beta}}), \ldots, H i t_{T}(\hat{\boldsymbol{\beta}})\right]^{\prime}
$$

Visando testes para fins de seleção de modelos e que também possam avaliar quando uma estimativa de $V a R$ apresentada a uma instituição financeira satisfaz 
alguns requisitos básicos, Engle e Manganelli (2004) desenvolveram os testes de Quantis Dinâmicos (QD), que podem ser definidos como amostrais $\left(Q D_{a m}\right)$ e pósamostrais $\left(Q D_{\text {pam }}\right)$.

Inicialmente, detalha-se o teste $Q D_{a m}$, o qual verifica a qualidade do ajuste permitindo a avaliação do modelo e possibilitando a escolha daquele mais adequado aos dados.

Seja

$$
\boldsymbol{M}_{T} \equiv\left(\boldsymbol{X}^{\prime}\left(\hat{\boldsymbol{\beta}}_{\theta}\right)\right)-E\left[\frac{1}{T} \boldsymbol{X}^{\prime}\left(\hat{\boldsymbol{\beta}}_{\theta}\right) H \nabla V a R(\theta)\right] \boldsymbol{D}_{T}^{-1} \times \nabla^{\prime} V a R(\theta),
$$

em que $\boldsymbol{H}$ é uma matriz diagonal, formada por $h_{t}\left(0 \mid \mathcal{F}_{t}\right)$.

Sob certas suposições, assumindo algumas condições de regularidade, Engle e Manganelli (2004) provaram que

$$
Q D_{a m} \equiv \frac{\boldsymbol{H} \boldsymbol{i t}(\hat{\boldsymbol{\beta}}) \boldsymbol{X}(\hat{\boldsymbol{\beta}})\left(\hat{\boldsymbol{M}}_{\boldsymbol{T}} \hat{\boldsymbol{M}}_{\boldsymbol{T}}^{\prime}\right)^{-1} \boldsymbol{X}^{\prime}(\hat{\boldsymbol{\beta}}) \boldsymbol{H} \boldsymbol{i t} \boldsymbol{t}^{\prime}(\hat{\boldsymbol{\beta}})}{\theta(1-\theta)} \stackrel{d}{\sim} \chi_{(k)}, \text { quando } T \rightarrow \infty,
$$

em que

$$
\begin{array}{r}
\hat{\boldsymbol{M}}_{T} \equiv \boldsymbol{X}^{\prime}(\hat{\boldsymbol{\beta}})-\left\{\left(2 T \hat{c}_{T}\right)^{-1} \sum_{t=1}^{T} I\left(\left|r_{t}-\widehat{\operatorname{VaR}}_{t}(\theta)\right|<\hat{c}_{T}\right) \times\right. \\
\left.\times \boldsymbol{x}_{t}^{\prime}(\hat{\boldsymbol{\beta}}) \nabla \widehat{V a R}_{t}(\theta)\right\} \hat{\boldsymbol{D}}_{T}^{-1} \nabla^{\prime} \widehat{\operatorname{VaR}}(\theta) .
\end{array}
$$

Já o teste de $Q D_{\text {pam }}$ serve como ferramenta para verificar se as estimativas dos parâmetros de interesse satisfazem alguns requisitos que toda boa estimativa quantílica deve satisfazer. Uma das grandes vantagens do teste é que pode ser considerado como simples, além do fato de não depender do procedimento de estimação. Para implementá-lo, é necessário a sequência de valores de $V a R s$ e dos correspondentes valores dos retornos.

Sendo assim, seja $T_{R}$ o número de observações amostrais e $N_{R}$ o número de observações pós-amostrais, ambos dependendo de $T$, em que $T \in \mathbb{Z}$. Assim, se $T$ é o 
número total de observações, então

$$
T=T_{R}+N_{R},
$$

em que, satisfazendo certas condições,

$$
\lim _{T \rightarrow \infty} T_{R}=\infty, \lim _{T \rightarrow \infty} N_{R}=\infty
$$

e

$$
\lim _{T \rightarrow \infty} \frac{N_{R}}{T_{R}}=0 .
$$

Seja o $k$-vetor mensurável $\mathcal{F}_{n}$, como sendo $\boldsymbol{X}_{n}\left(\hat{\boldsymbol{\beta}}_{T_{R}}\right)$, em que $n=T_{R}+1, \ldots, T_{R}+N_{R}$, uma linha da matriz $\boldsymbol{X}\left(\hat{\boldsymbol{\beta}}_{T_{R}}\right)$, dependendo de $\hat{\boldsymbol{\beta}}_{T_{R}}$, e considerando também

$$
\boldsymbol{H i t}\left(\hat{\boldsymbol{\beta}}_{T_{R}}\right) \equiv\left[H i t_{T_{R}+1}\left(\hat{\boldsymbol{\beta}}_{T_{R}}\right), H i t_{T_{R}+2}\left(\hat{\boldsymbol{\beta}}_{T_{R}}\right), \ldots, H i t_{T_{R}+N_{R}}\left(\hat{\boldsymbol{\beta}}_{T_{R}}\right)\right]^{\prime},
$$

então, sob certas suposições,

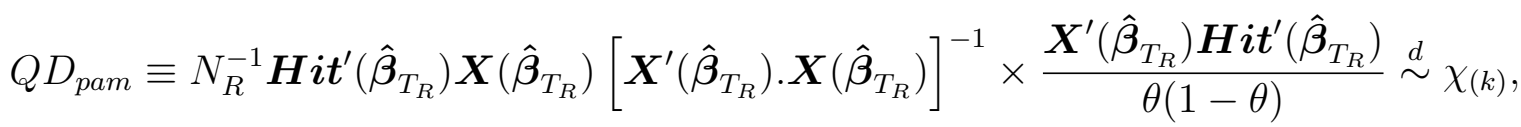
quando $T \rightarrow \infty$.

Como alternativa para verificar se a especificação escolhida é apropriada, Engle e Manganelli (2004) sugerem que se analise o percentual de valores que as funções indicadoras assumem na amostra, ou seja, escolhendo um $\theta$ apropriado, para posição vendida ou comprada, calcula-se

$$
p=\frac{1}{T} \sum_{t=1}^{T} I\left(r_{t}<\operatorname{Va} R_{t}(\theta)\right),
$$

assim, $p \approx \theta$ é um indicativo de que modelo é adequado.

Esta proporção, considerando $T_{R}$ e $N_{R}$ é definida como proporção de $H_{i t} t_{a m}$ e Hit $t_{\text {pam }}$. 
44CAPÍTULO 4. CAVIAR - VALOR EM RISCO AUTORREGRESSIVO CONDICIONAL 


\section{Capítulo 5}

\section{CARE - Expectil Autorregressivo Condicional}

\subsection{Conceitos}

Uma das fragilidades dos modelos CAViaR com parâmetros estimados por Regressão Quantílica é que a magnitude dos dados não tem influência no processo de estimação, pois o que se considera de fato são os quantis dos dados.

Assim, ao considerar por exemplo, dois conjuntos de dados com caudas se comportando de formas diferentes, eles podem apresentar o mesmo valor para um $\theta$-quantil.

Com o objetivo de obter medidas de risco calculadas por um método mais adequado, Taylor (2008) introduz os modelos Expectil Autorregressivo Condicional (CARE) com as mesmas estruturas dos modelos CAViaR, porém com parâmetros estimados por Mínimos Quadrados Assimétricos. Estes modelos receberam algumas críticas por apresentar as mesmas estruturas dos modelos CAViaR, não apresentar e nem discutir as propriedades assintóticas do estimador de Mínimos Quadrados Assimétricos, além de não definir um teste no contexto dinâmico.

Sendo assim, Kuan, Yeh e Hsu (2009) propõem novas especificações, com propriedades assintóticas e testes apresentados ao longo do Capítulo. 
Antes de detalhar as especificações do modelo, é necessário que se defina expectil.

\section{$5.2 \quad$ Expectil}

Considere a função de perda definida anteriormente $\rho_{\tau}(u)$, com $\tau \in(0,1)$ fixo. O $\tau$ - expectil, definido como $\mu(\tau)$ é o parâmetro $m$ que minimiza a função de perda calculada em $(Y-m)^{2}$, com relação à v.a. Y, ou seja, é um mínimo quadrado assimétrico (MQA), em que

$$
M Q A=\min _{m \in \mathbb{R}}\left[E\left\{\rho_{\tau}(Y-m)^{2}\right\}\right]
$$

De fato, para o cálculo de $\mu(\tau)$ de acordo com (5.1), tem -se:

$$
E\left\{\rho_{\tau}(Y-m)^{2}\right\}=|\tau-1| \int_{-\infty}^{m}(Y-m)^{2} d F_{Y}(y)+|\tau| \int_{m}^{+\infty}(Y-m)^{2} d F_{Y}(y) .
$$

Para minimizar, basta diferenciar com relação a m e igualar a zero, assim:

$$
(1-\tau) \int_{-\infty}^{\mu(\tau)}(Y-\mu(\tau)) d F_{Y}(y)+\tau \int_{\mu(\tau)}^{+\infty}(Y-\mu(\tau)) d F_{Y}(y)=0
$$

Fazendo

$$
\int_{\mu(\tau)}^{+\infty}(Y-\mu(\tau)) d F_{Y}(y)=E(Y-\mu(\tau))-\int_{-\infty}^{\mu(\tau)}(Y-\mu(\tau)) d F_{Y}(y)
$$

e substituindo (5.3) em (5.2), tem-se:

$$
(1-\tau) \int_{-\infty}^{\mu(\tau)}(Y-\mu(\tau)) d F_{Y}(y)+\tau\left[E(Y-\mu(\tau))-\int_{-\infty}^{\mu(\tau)}(Y-\mu(\tau)) d F_{Y}(y)\right]=0 \Rightarrow
$$




$$
\begin{aligned}
& \Rightarrow \quad(1-\tau) \int_{-\infty}^{\mu(\tau)}(Y-\mu(\tau)) d F_{Y}(y)+\tau[E(Y-\mu(\tau))]-\tau \int_{-\infty}^{\mu(\tau)}(Y-\mu(\tau)) d F_{Y}(y)=0 \\
& \Rightarrow \quad(1-2 \tau) \int_{-\infty}^{\mu(\tau)}(Y-\mu(\tau)) d F_{Y}(y)-\tau[\mu(\tau)-E(Y)]=0 \\
& \Rightarrow \quad(1-2 \tau) \int_{-\infty}^{\mu(\tau)}(Y-\mu(\tau)) d F_{Y}(y)=\tau[\mu(\tau)-E(Y)] \\
& \Rightarrow \mu(\tau)-E(Y)=\left(\frac{1-2 \tau}{\tau}\right) \int_{-\infty}^{\mu(\tau)}(Y-\mu(\tau)) d F_{Y}(y) .
\end{aligned}
$$

Assim, para um $\tau$ fixo,

$$
\mu(\tau)-E(Y)=\left(\frac{1-2 \tau}{\tau}\right) \int_{-\infty}^{\mu(\tau)}(Y-\mu(\tau)) d F_{Y}(y) .
$$

Logo, pode se concluir que o resultado dessa minimização é o valor $\mu(\tau)$ que satisfaz a equação (5.4).

Ao comparar o $\theta$-quantil e o $\tau$-expectil, Koenker (2005) observa que o expectil tem uma maior dependência global da forma da distribuição. Por exemplo, alterando a forma da distribuição nas caudas, o quantil não é alterado, mas há um impacto no expectil.

De acordo com a definição de expectil, inicialmente é necessário definir qual o valor mais adequado para $\tau$, dado o valor de $\theta$.

Seja $\mu(\tau)$, tal que, de acordo com a equação (5.4):

$$
\begin{aligned}
\mu(\tau)-E(Y) & =\left(\frac{1-2 \tau}{\tau}\right) \int_{-\infty}^{\mu(\tau)}(Y-\mu(\tau)) d F_{Y}(y) \\
\Rightarrow \mu(\tau)-E(Y) & =\left(\frac{1-2 \tau}{\tau}\right)\left\{E\left(Y I_{y<\mu(\tau)}\right)-\mu(\tau) E\left(I_{y<\mu(\tau)}\right)\right\} \\
\Rightarrow \frac{1}{\theta}(\mu(\tau)-E(Y)) & =\left(\frac{1-2 \tau}{\tau}\right) \frac{1}{\theta}\left\{E\left(Y I_{y<\mu(\tau)}\right)-\mu(\tau) E\left(I_{y<\mu(\tau)}\right)\right\} .
\end{aligned}
$$

Sendo assim, fazendo $E\left(I_{y<\mu(\tau)}\right)=F_{a c}(\mu(\tau))=\theta$, em que $F_{a c}$ é a função de 
distribuição acumulada da v.a. Y, tem-se:

$$
\begin{aligned}
\frac{1}{\theta}(\mu(\tau)-E(Y)) & =\left(\frac{1-2 \tau}{\tau}\right) \frac{1}{\theta}\left\{E\left(Y I_{y<\mu(\tau)}\right)-\mu(\tau) \theta\right\} \\
\Rightarrow \frac{\mu(\tau)-E(Y)}{\theta} & =\left(\frac{1-2 \tau}{\tau}\right)\left\{\frac{E\left(Y I_{y<\mu(\tau)}\right)}{\theta}-\mu(\tau)\right\} \\
\Rightarrow\left(\frac{1-2 \tau}{\tau}\right) & =\frac{\mu(\tau)-E(Y)}{\theta\left\{\frac{E\left(Y I_{y<\mu(\tau)}\right)}{\theta}-\mu(\tau)\right\}} .
\end{aligned}
$$

Estendendo o conceito para uma série de retornos, inicialmente, fixa-se o valor de $\theta$, logo $\hat{\mu}(\tau)=q_{\theta}\left(r_{t} \mid \mathcal{F}_{t-1}\right)$, em que $q_{\theta}\left(r_{t} \mid \mathcal{F}_{t-1}\right)$ é o $\theta-q u a n t i l$ empírico da série de retornos dadas as informações passadas, $E(Y)=E\left(r_{t} \mid \mathcal{F}_{t-1}\right)$, e

$$
\frac{E\left(Y I_{y<\mu(\tau)}\right)}{\theta}=E\left(\left(r_{t} \mid \mathcal{F}_{t-1}\right) \mid\left(r_{t} \mid \mathcal{F}_{t-1}\right)<q_{\theta}\left(r_{t} \mid \mathcal{F}_{t-1}\right)\right)
$$

ou seja, $\frac{E\left(Y I_{y<\hat{\mu}(\tau)}\right)}{\theta}$ é obtido pela média dos retornos abaixo do $\theta$ - quantil estimado.

Logo, de acordo com (5.5), tem-se:

$$
\frac{1-2 \tau}{\tau}=\frac{\hat{\mu}(\tau)-E\left(r_{t} \mid \mathcal{F}_{t-1}\right)}{\theta\left\{E\left(\left(r_{t} \mid \mathcal{F}_{t-1}\right) \mid\left(r_{t} \mid \mathcal{F}_{t-1}\right)<q_{\theta}\left(r_{t} \mid \mathcal{F}_{t-1}\right)\right)-\hat{\mu}(\tau)\right\}} .
$$

Fazendo o segundo lado da igualdade igual a $K$, tem-se:

$$
\hat{K}=\frac{\hat{\mu}(\tau)-E\left(r_{t} \mid \mathcal{F}_{t-1}\right)}{\theta\left\{E\left(\left(r_{t} \mid \mathcal{F}_{t-1}\right) \mid\left(r_{t} \mid \mathcal{F}_{t-1}\right)<q_{\theta}\left(r_{t} \mid \mathcal{F}_{t-1}\right)\right)-\hat{\mu}(\tau)\right\}} .
$$

Assim, substituindo (5.7) em (5.6), tem-se:

$$
\frac{1-2 \tau}{\tau}=\hat{K} \Rightarrow \hat{\tau}=\frac{\hat{K}}{1+2 \hat{K}}
$$

Seguindo as mesmas estruturas dos modelos CAViaR, e utilizando o $\tau$ - expectil como um estimador para o $\theta$-quantil, em que não necessariamente $\tau$ seja igual a $\theta$, Taylor (2008) define as seguintes especificações para $\mu_{\tau}$ : 


\subsection{Especificações dos modelos CARE}

Taylor (2008) propões as seguntes especificações:

5.3.1 Valor Absoluto Simétrico:

$$
\mu_{t}(\tau)=\beta_{1}+\beta_{2} \mu_{t-1}(\tau)+\beta_{3}\left|r_{t-1}\right|
$$

5.3.2 Inclinação Assimétrica:

$$
\mu_{t}(\tau)=\beta_{1}+\beta_{2} \mu_{t-1}(\tau)+\beta_{3}\left(r_{t-1}\right) I\left(r_{t-1}>0\right)+\beta_{4}\left(r_{t-1}\right) I\left(r_{t-1}<0\right) .
$$

5.3.3 GARCH(1,1) Indireto:

$$
\mu_{t}(\tau)=\left(\beta_{1}+\beta_{2} \mu_{t-1}^{2}(\tau)+\beta_{3} r_{t-1}^{2}\right)^{\frac{1}{2}}
$$

5.3.4 Adaptativo:

$$
\mu_{t}(\tau)=\mu_{t-1}(\tau)+\beta_{1}\left\{\left[1+\exp \left[k\left(r_{t-1}-\mu_{t-1}(\tau)\right)\right]\right]^{-1}-\theta\right\} .
$$

5.3.5 Adaptativo Simétrico Proporcional:

$$
\begin{aligned}
\mu_{t}(\tau)=\mu_{t-1}(\tau)+ & \beta_{1} \max \left\{0,\left|r_{t-1}\right|-\mu_{t-1}(\tau)\right\}- \\
& -\beta_{2} \min \left\{0,\left|r_{t-1}\right|-\mu_{t-1}(\tau)\right\} .
\end{aligned}
$$

\subsubsection{Valor Absoluto Assimétrico:}

$$
\mu_{t}(\tau)=\beta_{1}+\beta_{2} \mu_{t-1}(\tau)+\beta_{3}\left|r_{t-1}-\beta_{4}\right| .
$$


50 CAPÍTULO 5. CARE - EXPECTIL AUTORREGRESSIVO CONDICIONAL

\subsubsection{AR(1)-GARCH(1,1) Indireto:}

$$
\mu_{t}(\theta)=\phi_{1} r_{t-1}+\left[\beta_{1}+\beta_{2}\left(\mu_{t-1}(\theta)-\phi_{1} r_{t-2}\right)^{2}+\beta_{3}\left(r_{t-1}-\phi_{1} r_{t-2}\right)^{2}\right]^{\frac{1}{2}}
$$

As especificações possuem as mesmas características que as descritas e detalhadas para os modelos CAViaR.

Para a estimação dos modelos CARE, utiliza-se o mesmo procedimento que o feito para a estimação dos modelos CAViaR, porém ao invés de calcular a função de $C Q$ é calculada a função de $M Q A$.

Com objetivo de selecionar e verificar a adequação do modelo, como feito para os modelos CAViaR, Taylor (2008) propõe os testes de $H i t_{a m}, H i t_{p a m}$ e $Q D_{a m}$ e $Q D_{\text {pam }}$.

Kuan, Yeh e Hsu (2009) propõem novas especificações, mencionadas a seguir.

Considerando:

i) Uma coleção de $k$ variáveis, $\boldsymbol{X}$ o conjunto de informações, tais que $\boldsymbol{X} \in \mathcal{F}_{t}$ e $\boldsymbol{\beta}_{\boldsymbol{\tau}}$ o vetor $(k \times 1)$ de parâmetros dependendo de $\tau$;

ii) $\left(r_{t}, \boldsymbol{x}_{t}^{\prime}\right)$ o conjunto de dados disponível;

iii) o modelo:

$$
r_{t}=\boldsymbol{x}_{t}^{\prime} \boldsymbol{\beta}_{\boldsymbol{\tau}}+e_{t, \tau}
$$

em que $\boldsymbol{x}_{t}^{\prime} \in \boldsymbol{X}, t=\{1, \ldots, T\}$ e $e_{t, \tau}$ é o erro;

v) $\mu(\tau, \boldsymbol{X})$ o $\tau-$ expectil do conjunto de informações condicional em $\mathcal{F}$;

vi) o modelo está corretamente especificado para $\mu(\tau, \boldsymbol{X})$ se existe $\boldsymbol{\beta}_{\tau}^{0}$ tal que $\boldsymbol{X}^{\prime} \boldsymbol{\beta}_{\tau}^{0}=\mu(\tau, \boldsymbol{X})$ com probabilidade um;

Sob a suposição de que o modelo é corretamente especificado, tem-se:

$$
r_{t}=\boldsymbol{x}_{t}^{\prime} \boldsymbol{\beta}_{\tau}^{0}+\epsilon_{t, \tau}
$$


No contexto dinâmico, para modelar o $\tau$ - expectil de $r_{t}$, são consideradas informações até o instante $(t-1)$, ou seja, $\boldsymbol{x}_{t} \in \mathcal{F}_{t-1}$.

Sabendo que o expectil é também um quantil, se uma série é autocorrelacionada, $\mu(\tau)$ para valores fixos de $\tau$ também será autocorrelacionada. Visando ter uma medida de risco que não apresente este comportamento, retornos defasados no tempo são incluídos nos modelos.

De acordo com a definição de expectil, visando incorporar o impacto que a magnitude dos dados exercem no modelo, o quadrado ou o valor absoluto dos retornos também podem ser incluídos nos modelos.

Sejam

$$
\begin{aligned}
r_{t-1} & =r_{t-1}^{+}-r_{t-1}^{-} \\
\left|r_{t-1}\right| & =r_{t-1}^{+}+r_{t-1}^{-} \\
r_{t-1}^{2} & =\left(r_{t-1}^{+}\right)^{2}+\left(r_{t-1}^{-}\right)^{2},
\end{aligned}
$$

em que $r_{t-1}^{+}=\max \left(r_{t-1}, 0\right)$ e $r_{t-1}^{-}=\max \left(-r_{t-1}, 0\right)$.

Logo, as especificações CARE são mostradas a seguir, com

$\boldsymbol{x}_{t}=\left(1, r_{t-1},\left(r_{t-1}^{+}\right)^{2},\left(r_{t-1}^{-}\right)^{2}\right)^{\prime}$, de modo que, de acordo com (5.16) e (5.17) seguem as estruturas:

\subsubsection{Especificação [1]}

$$
\begin{aligned}
& r_{t}=a_{0}(\tau)+a_{1}(\tau) r_{t-1}+b_{1}(\tau) r_{t-1}^{2}+c_{1}(\tau)\left(r_{t-1}^{-}\right)^{2}+e_{t, \tau} \Rightarrow \\
& r_{t}=a_{0}(\tau)+a_{1}(\tau) r_{t-1}+b_{1}(\tau)\left[\left(r_{t-1}^{+}\right)^{2}+\left(r_{t-1}^{-}\right)^{2}\right]+c_{1}(\tau)\left(r_{t-1}^{-}\right)^{2}+e_{t, \tau} \Rightarrow \\
& r_{t}=a_{0}(\tau)+a_{1}(\tau) r_{t-1}+b_{1}(\tau)\left(r_{t-1}^{+}\right)^{2}+\gamma_{1}(\tau)\left(r_{t-1}^{-}\right)^{2}+e_{t, \tau},
\end{aligned}
$$

em que $\gamma_{1}(\tau)=b_{1}(\tau)+c_{1}(\tau)$.

Neste caso,

$$
\begin{aligned}
& \mu_{t}(\tau)=a_{0}(\tau)+a_{1}(\tau)\left[\mu_{t-1}(\tau)+e_{t-1, \tau}\right]+b_{1}(\tau)\left(r_{t-1}^{+}\right)^{2}+\gamma_{1}(\tau)\left(r_{t-1}^{-}\right)^{2} \Rightarrow \\
& \mu_{t}(\tau)=a_{0}(\tau)+a_{1}(\tau) \mu_{t-1}(\tau)+b_{1}(\tau)\left(r_{t-1}^{+}\right)^{2}+\gamma_{1}(\tau)\left(r_{t-1}^{-}\right)^{2}+a_{1}(\tau) e_{t-1, \tau} .
\end{aligned}
$$


Neste modelo, a magnitude e o erro defasado no tempo são responsáveis pelo comportamento do expectil condicional do modelo.

Note que, $\boldsymbol{\beta}_{\tau}=\left(a_{0}(\tau), a_{1}(\tau), b_{1}(\tau), \gamma_{1}(\tau)\right)$.

\subsubsection{Especificação [2]}

Uma outra alternativa de especificação, é o uso do $\left|r_{t-1}\right|$ para representar o efeito dos dados no modelo.

Assim,

$$
\begin{aligned}
& r_{t}=a_{0}(\tau)+a_{1}(\tau) r_{t-1}+b_{1}(\tau)\left|r_{t-1}\right|+e_{t, \tau} \Rightarrow \\
& r_{t}=a_{0}(\tau)+\gamma_{1}(\tau) r_{t-1}^{+}+\delta_{1}(\tau) r_{t-1}^{-}+e_{t, \tau},
\end{aligned}
$$

em que $\gamma_{1}(\tau)=a_{1}(\tau)+b_{1}(\tau)$ e $\delta_{1}(\tau)=b_{1}(\tau)-a_{1}(\tau)$.

Neste caso, $\boldsymbol{\beta}_{\tau}=\left(a_{0}(\tau), \gamma_{1}(\tau), \delta_{1}(\tau)\right)$.

Esta especificaçãó é similar a especificação do modelo CAViaR inclinação assimétrica, porém no modelo CARE o $\tau$ - expectil condicional defasado no tempo não está inserido no modelo.

\subsubsection{Especificação [3]}

Esta especificação, é na realidade uma estensão natural do modelo definido em (5.18).

Seja

$$
r_{t}=a_{0}(\tau)+\sum_{i=1}^{p} a_{i}(\tau) r_{t-i}+\sum_{j=1}^{q}\left[b_{j}(\tau)\left(r_{t-j}^{+}\right)^{2}+\gamma_{j}(\tau)\left(r_{t-j}^{-}\right)^{2}\right]+e_{t, \tau},
$$

neste caso,

$$
\mu_{t}(\tau)=a_{0}(\tau)+\sum_{i=1}^{p} a_{i}(\tau) \mu_{t-i}(\tau)+\sum_{j=1}^{q}\left[b_{j}(\tau)\left(r_{t-j}^{+}\right)^{2}+\gamma_{j}(\tau)\left(r_{t-j}^{-}\right)^{2}\right]+\sum_{i=1}^{p} e_{t-i, \tau}
$$


em que de forma análoga ao modelo (5.18), a magnitude dos dados e os erros defasados no tempo que determinam o comportamento do $\tau$-expectil, em que:

$$
\boldsymbol{\beta}_{\tau}=\left(a_{0}(\tau), a_{1}(\tau), \ldots, a_{p}(\tau), b_{1}(\tau), \ldots, b_{q}(\tau), \gamma_{1}(\tau), \ldots, \gamma_{q}(\tau)\right) .
$$

\subsubsection{Especificação [4]}

Como no caso anterior, esta é uma estensão do modelo (5.19).

Assim:

$$
r_{t}=a_{0}(\tau)+\sum_{i=1}^{p}\left[b_{i}(\tau) r_{t-i}^{+}+\gamma_{i}(\tau) r_{t-i}^{-}\right]+e_{t, \tau}
$$

$\operatorname{com} \boldsymbol{\beta}_{\tau}=\left(a_{0}(\tau), b_{1}(\tau), \ldots, b_{p}(\tau), \gamma_{1}(\tau), \ldots, \gamma_{p}(\tau)\right)$

\subsection{Estimação do Modelo}

As quatro especificações detalhadas anteriormente podem ser estimadas utilizando o método de Mínimos Quadrados Assimétricos (MQA), descrito e proposto por Newey e Powell (1987).

Neste caso a média da função de perda $\rho_{\tau}$ é calculada utilizando uma diferença quadrática $(y-m)^{2}$, dando pesos $(1-\tau)$ e $\tau$ de acordo com os sinais das diferenças, calculando o seu mínimo, como visto em (5.1). Por causa desses pesos que o método é chamado de assimétrico.

Assim, o estimador $\hat{\boldsymbol{\beta}}_{\tau}$ é o vetor $\boldsymbol{\beta}$ que satisfaz

$$
M Q A=\min _{\boldsymbol{\beta}}\left\{T^{-1} \sum_{t=1}^{T} \rho_{\tau}\left(r_{t}-\boldsymbol{x}_{t}^{\prime} \boldsymbol{\beta}\right)^{2}\right\} .
$$

Este estimador satisfaz

$$
\hat{\boldsymbol{\beta}}_{\tau}=\left(\sum_{t=1}^{T}\left|\tau-I\left(r_{t} \leq \boldsymbol{x}_{t}^{\prime} \hat{\boldsymbol{\beta}}_{\tau}\right)\right|\left(\boldsymbol{x}_{t} r_{t}\right)\right)\left(\sum_{t=1}^{T}\left|\tau-I\left(r_{t} \leq \boldsymbol{x}_{t}^{\prime} \hat{\boldsymbol{\beta}}_{\tau}\right)\right|\left(\boldsymbol{x}_{t} \boldsymbol{x}_{t}^{\prime}\right)\right)^{-1}
$$


Embora (5.23) não seja uma solução fechada, pode ser calculada como um estimador de mínimos quadrados ponderados iterado.

Newey e Powell (1987) estabelecem a consistência e normalidade assintótica do estimador dado em (5.22) sob as condições de que os dados são i.i.d.. De fato, mostram que

$$
\hat{\boldsymbol{\beta}}_{\tau} \stackrel{p}{\longrightarrow} \boldsymbol{\beta}_{\tau}, T \longrightarrow \infty,
$$

e também,

$$
\sqrt{T}\left(\hat{\boldsymbol{\beta}}_{\tau}-\boldsymbol{\beta}_{\tau}\right) \stackrel{d}{\longrightarrow} N(0, \boldsymbol{\Sigma}(\tau)),
$$

em que

$$
\boldsymbol{\Sigma}(\tau)=\boldsymbol{A}(\tau)^{-1} \boldsymbol{V}(\tau) \boldsymbol{A}(\tau)^{-1},
$$

com

$$
\begin{aligned}
& \boldsymbol{A}(\tau)=E\left[\left|\tau-I\left(r_{t} \leq \boldsymbol{x}_{t}^{\prime} \boldsymbol{\beta}_{\tau}\right)\right| \boldsymbol{x}_{t} \boldsymbol{x}_{t}^{\prime}\right] \\
& \boldsymbol{V}(\tau)=\lim _{T \rightarrow \infty} \boldsymbol{V}_{T}(\tau):=\lim _{T \rightarrow \infty}\left(\frac{1}{\sqrt{T}} \sum_{t=1}^{T}\left|\tau-I\left(r_{t} \leq \boldsymbol{x}_{t}^{\prime} \boldsymbol{\beta}_{\tau}\right)\right| \boldsymbol{x}_{t}\left(r_{t}-\boldsymbol{x}_{t}^{\prime} \boldsymbol{\beta}_{\tau}\right)\right) .
\end{aligned}
$$

De acordo com (5.16) e (5.17), se o modelo estiver corretamente especificado, $\boldsymbol{\beta}_{\tau}=\boldsymbol{\beta}_{\tau}^{0}$, onde

$$
\boldsymbol{\beta}_{\tau}^{0}=E\left[\rho_{\tau}\left(r_{t}-\boldsymbol{x}_{t}^{\prime} \boldsymbol{\beta}_{\tau}\right)^{2} \mid \mathcal{F}_{t-1}\right] .
$$

Considerando todas as condições de regularidade e as propriedades de diferenças martingales, Newey e Powell (1987) mostram que

$$
\sqrt{T}\left(\hat{\boldsymbol{\beta}}_{\tau}-\boldsymbol{\beta}_{\tau}^{0}\right) \stackrel{d}{\longrightarrow} N(0, \boldsymbol{\Sigma}(\tau)),
$$

em que

$$
\boldsymbol{\Sigma}(\tau)=\boldsymbol{A}(\tau)^{-1} \boldsymbol{V}(\tau) \boldsymbol{A}(\tau)^{-1},
$$


com

$$
\boldsymbol{V}(\tau)=\operatorname{Var}\left(\left|\tau-I\left(r_{t} \leq \boldsymbol{x}_{t}^{\prime} \boldsymbol{\beta}_{\tau}^{0}\right)\right| \boldsymbol{x}_{t}^{\prime}\left(r_{t}-\boldsymbol{x}_{t}^{\prime} \boldsymbol{\beta}_{\tau}^{0}\right)\right)
$$

A matriz de covariância assintótica $\boldsymbol{\Sigma}(\tau)$ pode ser estimada por

$$
\hat{\Sigma}(\tau)=\hat{\boldsymbol{A}}(\tau)^{-1} \hat{\boldsymbol{V}}(\tau) \hat{\boldsymbol{A}}(\tau)^{-1}
$$

em que

$$
\begin{aligned}
\hat{\boldsymbol{A}}(\tau) & =\frac{1}{T} \sum_{t=1}^{T}\left(\left|\tau-I\left(r_{t} \leq \boldsymbol{x}_{t}^{\prime} \hat{\boldsymbol{\beta}}_{\tau}\right)\right| \boldsymbol{x}_{t} \boldsymbol{x}_{t}^{\prime}\right) \stackrel{\mathbb{P}}{\longrightarrow} \boldsymbol{A}(\tau) \\
\hat{\boldsymbol{V}}(\tau) & =\frac{1}{T} \sum_{t=1}^{T}\left(\left|\tau-I\left(r_{t} \leq \boldsymbol{x}_{t}^{\prime} \hat{\boldsymbol{\beta}}_{\tau}\right)\right|^{2}\left(r_{t}-\boldsymbol{x}_{t}^{\prime} \hat{\boldsymbol{\beta}}_{\tau}\right)^{2} \boldsymbol{x}_{t} \boldsymbol{x}_{t}^{\prime}\right) \stackrel{\mathbb{P}}{\longrightarrow} \boldsymbol{V}(\tau),
\end{aligned}
$$

com

$$
\boldsymbol{V}(\tau)=\operatorname{Var}\left\{\left|\tau-I\left(r_{t} \leq \boldsymbol{x}_{t}^{\prime} \boldsymbol{\beta}_{\tau}^{0}\right)\right| \boldsymbol{x}_{t}\left(r_{t}-\boldsymbol{x}_{t}^{\prime} \boldsymbol{\beta}_{\tau}^{0}\right)\right\}
$$

\subsection{Teste de Ajuste dos Modelos}

Pelas especificações escolhidas, de acordo com os autores, para determinar qual o modelo mais apropriado, foi desenvolvido um teste com as seguintes hipóteses:

$$
\left\{\begin{array}{l}
H_{0}: \boldsymbol{x}_{t}^{\prime} \boldsymbol{\beta}_{\tau}^{0}=\mu\left(\tau, \mathcal{F}_{t-1}\right), \text { com probabilidade } 1 \\
H_{1}: \boldsymbol{\zeta}_{t}^{\prime} \boldsymbol{\beta}_{\tau}^{0}=\mu\left(\tau, \mathcal{F}_{t-1}\right), \text { com probabilidade } 1
\end{array}\right.
$$

em que $\boldsymbol{x}_{\boldsymbol{t}}$ tem ordem $(k \times T)$ e $\boldsymbol{\zeta}_{\boldsymbol{t}}$ com ordem $(m \times T)$, ambos contidos em $\mathcal{F}_{t-1}$, onde $k=3 q+1$ e $m=2 q+1$.

Note que

$$
\begin{aligned}
\boldsymbol{x}_{\boldsymbol{t}}^{\prime} & =\left(1, r_{t-i},\left(r_{t-i}^{+}\right)^{2},\left(r_{t-i}^{-}\right)^{2}\right), i=1, \ldots, q \mathrm{e} \\
\boldsymbol{\zeta}_{\boldsymbol{t}}^{\prime} & =\left(1, r_{t-i}^{+}, r_{t-i}^{-}\right), i=1, \ldots, q .
\end{aligned}
$$


De acordo com os autores, uma forma de testar o modelo sob a hipótese nula, é checar se os erros ponderados do modelo sob a hipótese nula são não correlacionados com as variáveis do modelo sob a hipótese alternativa, ou seja:

$$
E\left[\boldsymbol{\zeta}_{t}\left|\tau-I\left(r_{t} \leq \boldsymbol{x}_{t}^{\prime} \boldsymbol{\beta}_{\tau}^{0}\right)\right|\left(r_{t}-\boldsymbol{x}_{t}^{\prime} \boldsymbol{\beta}_{\tau}^{0}\right)\right]=\mathbf{0} .
$$

Demonstração:

Estendendo o conceito para uma série de retornos, logo:

$$
E\left[\boldsymbol{\zeta}_{t}\left|\tau-I\left(r_{t} \leq \boldsymbol{x}_{t}^{\prime} \boldsymbol{\beta}_{\tau}^{0}\right)\right|\left(r_{t}-\boldsymbol{x}_{t}^{\prime} \boldsymbol{\beta}_{\tau}^{0}\right)\right]=\frac{1}{\sqrt{T}} \sum_{t=1}^{T} \boldsymbol{\zeta}_{t}\left|\tau-I\left(r_{t} \leq \boldsymbol{x}_{t}^{\prime} \hat{\boldsymbol{\beta}}_{\tau}\right)\right| \hat{e}_{t, \tau}
$$

assim,

$$
E\left[\boldsymbol{\zeta}_{t}\left|\tau-I\left(r_{t} \leq \boldsymbol{x}_{t}^{\prime} \boldsymbol{\beta}_{\tau}^{0}\right)\right|\left(r_{t}-\boldsymbol{x}_{t}^{\prime} \boldsymbol{\beta}_{\tau}^{0}\right)\right]=\mathbf{0},
$$

onde, por (5.16) e (5.17),

$$
\hat{e}_{t, \tau}=\boldsymbol{x}_{t}^{\prime} \boldsymbol{\beta}_{\tau}^{0}-\epsilon_{t, \tau}-\boldsymbol{x}_{t}^{\prime} \hat{\boldsymbol{\beta}}_{\tau} .
$$

De acordo com (5.24) e (5.25), tem-se:

$$
\begin{aligned}
\frac{1}{\sqrt{T}} \sum_{t=1}^{T} \boldsymbol{\zeta}_{t}\left|\tau-I\left(r_{t} \leq \boldsymbol{x}_{t}^{\prime} \hat{\boldsymbol{\beta}}_{\tau}\right)\right| \hat{e}_{t, \tau} & =\frac{1}{\sqrt{T}} \sum_{t=1}^{T} \boldsymbol{\zeta}_{t}\left|\tau-I\left(r_{t} \leq \boldsymbol{x}_{t}^{\prime} \hat{\boldsymbol{\beta}}_{\tau}\right)\right| \epsilon_{t, \tau}- \\
& -\frac{1}{T} \sum_{t=1}^{T}\left|\tau-I\left(r_{t} \leq \boldsymbol{x}_{t}^{\prime} \hat{\boldsymbol{\beta}}_{\tau}\right)\right| \boldsymbol{\zeta}_{t} \boldsymbol{x}_{t}^{\prime} \sqrt{T}\left(\hat{\boldsymbol{\beta}}_{\tau}-\boldsymbol{\beta}_{\tau}^{0}\right) .
\end{aligned}
$$

Por (5.23),

$$
\begin{aligned}
\sqrt{T}\left(\hat{\boldsymbol{\beta}}_{\tau}-\boldsymbol{\beta}_{\tau}^{0}\right) & =\sqrt{T}\left\{\left[\sum_{t=1}^{T}\left|\tau-I\left(r_{t} \leq \boldsymbol{x}_{t}^{\prime} \hat{\boldsymbol{\beta}}_{\tau}\right)\right|\left(\boldsymbol{x}_{t} r_{t}\right)\right] \times\right. \\
& \left.\times\left[\sum_{t=1}^{T}\left|\tau-I\left(r_{t} \leq \boldsymbol{x}_{t}^{\prime} \hat{\boldsymbol{\beta}}_{\tau}\right)\right|\left(\boldsymbol{x}_{t} \boldsymbol{x}_{t}^{\prime}\right)\right]^{-1}-\boldsymbol{\beta}_{\tau}^{0}\right\}
\end{aligned}
$$




$$
\begin{aligned}
& =\sqrt{T}\left\{\left[\sum_{t=1}^{T}\left|\tau-I\left(r_{t} \leq \boldsymbol{x}_{t}^{\prime} \hat{\boldsymbol{\beta}}_{\tau}\right)\right|\left(\boldsymbol{x}_{t} r_{t}\right)\right]\left[\sum_{t=1}^{T}\left|\tau-I\left(r_{t} \leq \boldsymbol{x}_{t}^{\prime} \hat{\boldsymbol{\beta}}_{\tau}\right)\right|\left(\boldsymbol{x}_{t} \boldsymbol{x}_{t}^{\prime}\right)\right]^{-1}-\right. \\
& \left.-\boldsymbol{\beta}_{\tau}^{0}\left[\sum_{t=1}^{T}\left|\tau-I\left(\boldsymbol{x}_{t} \leq \boldsymbol{x}_{t}^{\prime} \hat{\boldsymbol{\beta}}_{\tau}\right)\right|\left(\boldsymbol{x}_{t} \boldsymbol{x}_{t}^{\prime}\right)\right]\left[\sum_{t=1}^{T}\left|\tau-I\left(\boldsymbol{x}_{t} \leq \boldsymbol{x}_{t}^{\prime} \hat{\boldsymbol{\beta}}_{\tau}\right)\right|\left(\boldsymbol{x}_{t} \boldsymbol{x}_{t}^{\prime}\right)\right]^{-1}\right\} \\
& =\sqrt{T}\left\{\left[\sum_{t=1}^{T}\left|\tau-I\left(r_{t} \leq \boldsymbol{x}_{t}^{\prime} \hat{\boldsymbol{\beta}}_{\tau}\right)\right|\left(\boldsymbol{x}_{t} r_{t}\right)\right]-\boldsymbol{\beta}_{\tau}^{0}\left[\sum_{t=1}^{T}\left|\tau-I\left(r_{t} \leq \boldsymbol{x}_{t}^{\prime} \hat{\boldsymbol{\beta}}_{\tau}\right)\right|\left(\boldsymbol{x}_{t} \boldsymbol{x}_{t}^{\prime}\right)\right]\right\} \times \\
& \times \quad\left(\sum_{t=1}^{T}\left|\tau-I\left(r_{t} \leq \boldsymbol{x}_{t}^{\prime} \hat{\boldsymbol{\beta}}_{\tau}\right)\right|\left(\boldsymbol{x}_{t} \boldsymbol{x}_{t}^{\prime}\right)\right)^{-1} \cdot \\
& =\sqrt{T}\left(\sum_{t=1}^{T}\left|\tau-I\left(r_{t} \leq \boldsymbol{x}_{t}^{\prime} \hat{\boldsymbol{\beta}}_{\tau}\right)\right|\left(\boldsymbol{x}_{t} \boldsymbol{x}_{t}^{\prime}\right)\right)^{-1}\left(\sum_{t=1}^{T}\left|\tau-I\left(r_{t} \leq \boldsymbol{x}_{t}^{\prime} \hat{\boldsymbol{\beta}}_{\tau}\right)\right| \boldsymbol{x}_{t}\left(r_{t}-\boldsymbol{x}_{t}^{\prime} \boldsymbol{\beta}_{\tau}^{0}\right)\right) \\
& =\left(\frac{1}{T} \sum_{t=1}^{T}\left|\tau-I\left(r_{t} \leq \boldsymbol{x}_{t}^{\prime} \hat{\boldsymbol{\beta}}_{\tau}\right)\right|\left(\boldsymbol{x}_{t} \boldsymbol{x}_{t}^{\prime}\right)\right)^{-1}\left(\frac{1}{\sqrt{T}} \sum_{t=1}^{T}\left|\tau-I\left(r_{t} \leq \boldsymbol{x}_{t}^{\prime} \hat{\boldsymbol{\beta}}_{\tau}\right)\right|\left(\boldsymbol{x}_{t} \epsilon_{t, \tau}\right)\right) .
\end{aligned}
$$

Assim,

$$
\begin{aligned}
\frac{1}{\sqrt{T}} \sum_{t=1}^{T} \boldsymbol{\zeta}_{t}\left|\tau-I\left(r_{t} \leq \boldsymbol{x}_{t}^{\prime} \hat{\boldsymbol{\beta}}_{\tau}\right)\right| \hat{e}_{t, \tau} & =\left(\frac{1}{\sqrt{T}} \sum_{t=1}^{T} \boldsymbol{\zeta}_{t}\left|\tau-I\left(r_{t} \leq \boldsymbol{x}_{t}^{\prime} \hat{\boldsymbol{\beta}}_{\tau}\right)\right| \epsilon_{t, \tau}\right)- \\
& -\left(\frac{1}{T} \sum_{t=1}^{T}\left|\tau-I\left(r_{t} \leq \boldsymbol{x}_{t}^{\prime} \hat{\boldsymbol{\beta}}_{\tau}\right)\right| \boldsymbol{\zeta}_{t} \boldsymbol{x}_{t}^{\prime}\right) \times
\end{aligned}
$$$$
\times\left(\frac{1}{T} \sum_{t=1}^{T}\left|\tau-I\left(r_{t} \leq \boldsymbol{x}_{t}^{\prime} \hat{\boldsymbol{\beta}}_{\tau}\right)\right|\left(\boldsymbol{x}_{t} \boldsymbol{x}_{t}^{\prime}\right)\right)^{-1}\left(\frac{1}{\sqrt{T}} \sum_{t=1}^{T}\left|\tau-I\left(r_{t} \leq \boldsymbol{x}_{t}^{\prime} \hat{\boldsymbol{\beta}}_{\tau}\right)\right|\left(\boldsymbol{x}_{t} \epsilon_{t, \tau}\right)\right)
$$

E, por Newey e Powell (1987),

$$
\left|\frac{1}{T} \sum_{t=1}^{T}\right| \tau-I\left(r_{t} \leq \boldsymbol{x}_{t}^{\prime} \hat{\boldsymbol{\beta}}_{\tau}\right)\left|\left(\boldsymbol{x}_{t} \boldsymbol{x}_{t}^{\prime}\right)-\frac{1}{T} \sum_{t=1}^{T}\right| \tau-I\left(r_{t} \leq \boldsymbol{x}_{t}^{\prime} \boldsymbol{\beta}_{\tau}^{0}\right)\left|\left(\boldsymbol{x}_{t} \boldsymbol{x}_{t}^{\prime}\right)\right| \stackrel{\mathbb{P}}{\longrightarrow} \mathbf{0},
$$


e também,

$$
\left|\frac{1}{T} \sum_{t=1}^{T}\right| \tau-I\left(r_{t} \leq \boldsymbol{x}_{t}^{\prime} \hat{\boldsymbol{\beta}}_{\tau}\right)\left|\left(\boldsymbol{\zeta}_{t} \boldsymbol{x}_{t}^{\prime}\right)-\frac{1}{T} \sum_{t=1}^{T}\right| \tau-I\left(r_{t} \leq \boldsymbol{x}_{t}^{\prime} \boldsymbol{\beta}_{\tau}^{0}\right)\left|\left(\boldsymbol{\zeta}_{t} \boldsymbol{x}_{t}^{\prime}\right)\right| \stackrel{\mathbb{P}}{\longrightarrow} \mathbf{0} .
$$

Pela lei dos grande números, tem-se que:

$$
\frac{1}{T} \sum_{t=1}^{T}\left|\tau-I\left(r_{t} \leq \boldsymbol{x}_{t}^{\prime} \boldsymbol{\beta}_{\tau}^{0}\right)\right|\left(\boldsymbol{x}_{t} \boldsymbol{x}_{t}^{\prime}\right) \stackrel{\mathbb{P}}{\longrightarrow} \boldsymbol{A}(\tau)
$$

e

$$
\frac{1}{T} \sum_{t=1}^{T}\left|\tau-I\left(r_{t} \leq \boldsymbol{x}_{t}^{\prime} \boldsymbol{\beta}_{\tau}^{0}\right)\right|\left(\boldsymbol{\zeta}_{t} \boldsymbol{x}_{t}^{\prime}\right) \stackrel{\mathbb{P}}{\longrightarrow} E\left[\left|\tau-I\left(r_{t} \leq \boldsymbol{x}_{t}^{\prime} \boldsymbol{\beta}_{\tau}^{0}\right)\right|\left(\boldsymbol{\zeta}_{t} \boldsymbol{x}_{t}^{\prime}\right)\right]=: \boldsymbol{\Gamma}(\tau)
$$

Sendo assim,

$$
\begin{gathered}
\frac{1}{\sqrt{T}} \sum_{t=1}^{T} \boldsymbol{\zeta}_{t}\left|\tau-I\left(r_{t} \leq \boldsymbol{x}_{t}^{\prime} \hat{\boldsymbol{\beta}}_{\tau}\right)\right| \hat{e}_{t, \tau}= \\
=\frac{1}{\sqrt{T}} \sum_{t=1}^{T} \boldsymbol{\zeta}_{t}\left|\tau-I\left(r_{t} \leq \boldsymbol{x}_{t}^{\prime} \hat{\boldsymbol{\beta}}_{\tau}\right)\right| \epsilon_{t, \tau}-\boldsymbol{\Gamma}(\tau)(\boldsymbol{A}(\tau))^{-1}\left(\frac{1}{\sqrt{T}}\left|\tau-I\left(r_{t} \leq \boldsymbol{x}_{t}^{\prime} \hat{\boldsymbol{\beta}}_{\tau}\right)\right| \boldsymbol{x}_{t} \epsilon_{t, \tau}\right)= \\
=\frac{1}{\sqrt{T}} \sum_{t=1}^{T}\left[\boldsymbol{\zeta}_{t}-\boldsymbol{\Gamma}(\tau)(\boldsymbol{A}(\tau))^{-1} \boldsymbol{x}_{t}\right]\left(\left|\tau-I\left(r_{t} \leq \boldsymbol{x}_{t}^{\prime} \hat{\boldsymbol{\beta}}_{\tau}\right)\right| \epsilon_{t, \tau}\right)+o_{\mathbb{P}}(1) .
\end{gathered}
$$

Logo, sob a hipótese nula,

$$
\begin{gathered}
\frac{1}{\sqrt{T}} \sum_{t=1}^{T} \boldsymbol{\zeta}_{t}\left|\tau-I\left(r_{t} \leq \boldsymbol{x}_{t}^{\prime} \hat{\boldsymbol{\beta}}_{\tau}\right)\right| \hat{e}_{t, \tau}= \\
\frac{1}{\sqrt{T}} \sum_{t=1}^{T}\left[\boldsymbol{\zeta}_{t}-\boldsymbol{\Gamma}(\tau)(\boldsymbol{A}(\tau))^{-1} \boldsymbol{x}_{t}\right]\left(\left|\tau-I\left(r_{t} \leq \boldsymbol{x}_{t}^{\prime} \boldsymbol{\beta}_{\tau}^{0}\right)\right| \epsilon_{t, \tau}\right)+o_{\mathbb{P}}(1) \stackrel{D}{\longrightarrow} \mathcal{N}(\mathbf{0}, \boldsymbol{\Omega}(\tau)),
\end{gathered}
$$

com

$$
\begin{array}{r}
\boldsymbol{\Omega}(\tau)=E\left[\left(\left|\tau-I\left(r_{t} \leq \boldsymbol{x}_{t}^{\prime} \boldsymbol{\beta}_{\tau}^{0}\right)\right|\right)^{2}\left(\epsilon_{t, \tau}\right)^{2}\left(\boldsymbol{\zeta}_{t}-\boldsymbol{\Gamma}(\tau)(\boldsymbol{A}(\tau))^{-1} \boldsymbol{x}_{t}\right) \times\right. \\
\left.\times\left(\boldsymbol{\zeta}_{t}-\boldsymbol{\Gamma}(\tau)(\boldsymbol{A}(\tau))^{-1} \boldsymbol{x}_{t}\right)^{\prime}\right],
\end{array}
$$


onde $\boldsymbol{\Gamma}(\tau)$ tem ordem $q$. Sendo assim, sabendo que $\boldsymbol{\zeta}_{\boldsymbol{t}}$ tem ordem $(m \times T), q \leq m$, em que $q$ é o número de elementos que estão em $\boldsymbol{\zeta}_{\boldsymbol{t}}$ que não foram incluídos em $\boldsymbol{x}_{\boldsymbol{t}}$.

Considerando o fato de que

$$
\frac{1}{\sqrt{T}} \sum_{t=1}^{T} \boldsymbol{\zeta}_{t}\left|\tau-I\left(r_{t} \leq \boldsymbol{x}_{t}^{\prime} \hat{\boldsymbol{\beta}}_{\tau}\right)\right| \hat{e}_{t, \tau} \stackrel{D}{\longrightarrow} \mathcal{N}(\mathbf{0}, \boldsymbol{\Omega}(\tau)),
$$

então

$$
\frac{1}{T}\left(\sum_{t=1}^{T}\left|\tau-I\left(r_{t} \leq \boldsymbol{x}_{t}^{\prime} \hat{\boldsymbol{\beta}}_{\tau}\right)\right| \boldsymbol{\zeta}_{t} \hat{e}_{t, \tau}\right)^{\prime}(\hat{\boldsymbol{\Omega}}(\tau))^{-1}\left(\sum_{t=1}^{T}\left|\tau-I\left(r_{t} \leq \boldsymbol{x}_{t}^{\prime} \hat{\boldsymbol{\beta}}_{\tau}\right)\right| \boldsymbol{\zeta}_{t} \hat{e}_{t, \tau}\right) \stackrel{D}{\longrightarrow} \chi_{(q)}^{2},
$$

onde $(\hat{\Omega}(\tau))^{-1}$ é a inversa generalizada do estimador consistente $\hat{\Omega}(\tau)$ para $\boldsymbol{\Omega}(\tau)$, que pode ser obtido por:

$$
\begin{gathered}
\hat{\boldsymbol{\Omega}}(\tau)=\frac{1}{T} \sum_{t=1}^{T}\left\{\left(\left|\tau-I\left(r_{t} \leq \boldsymbol{x}_{t}^{\prime} \hat{\boldsymbol{\beta}}_{\tau}\right)\right|^{2} \hat{e}_{t, \tau}^{2}\right)\right. \\
\times\left[\boldsymbol{\zeta}_{t}-\left(\frac{1}{T} \sum_{t=1}^{T}\left|\tau-I\left(r_{t} \leq \boldsymbol{x}_{t}^{\prime} \hat{\boldsymbol{\beta}}_{\tau}\right)\right| \boldsymbol{\zeta}_{t} \boldsymbol{x}_{t}^{\prime}\right)\left(\frac{1}{T} \sum_{t=1}^{T}\left|\tau-I\left(r_{t} \leq \boldsymbol{x}_{t}^{\prime} \hat{\boldsymbol{\beta}}_{\tau}\right)\right| \boldsymbol{x}_{t} \boldsymbol{x}_{t}^{\prime}\right)^{-1} \boldsymbol{x}_{t}\right] \\
\left.\times\left[\boldsymbol{\zeta}_{t}-\left(\frac{1}{T} \sum_{t=1}^{T}\left|\tau-I\left(r_{t} \leq \boldsymbol{x}_{t}^{\prime} \hat{\boldsymbol{\beta}}_{\tau}\right)\right| \boldsymbol{\zeta}_{t} \boldsymbol{x}_{t}^{\prime}\right)\left(\frac{1}{T} \sum_{t=1}^{T}\left|\tau-I\left(r_{t} \leq \boldsymbol{x}_{t}^{\prime} \hat{\boldsymbol{\beta}}_{\tau}\right)\right| \boldsymbol{x}_{t} \boldsymbol{x}_{t}^{\prime}\right)^{-1} \boldsymbol{x}_{t}\right]^{\prime}\right\} .
\end{gathered}
$$

De forma geral, considerando $\widetilde{\boldsymbol{\zeta}}_{t}$ um sub-vetor de $\boldsymbol{\zeta}_{t}$, não linear no espaço gerado pelas variáveis em $\boldsymbol{x}_{t}$, de acordo com as idéias mostradas anteriormente,

$$
\frac{1}{T}\left(\sum_{t=1}^{T}\left|\tau-I\left(r_{t} \leq \boldsymbol{x}_{t}^{\prime} \hat{\boldsymbol{\beta}}_{\tau}\right)\right| \widetilde{\boldsymbol{\zeta}}_{t} \hat{e}_{t, \tau}\right)^{\prime}(\widetilde{\boldsymbol{\Omega}}(\tau))^{-1}\left(\sum_{t=1}^{T}\left|\tau-I\left(r_{t} \leq \boldsymbol{x}_{t}^{\prime} \hat{\boldsymbol{\beta}}_{\tau}\right)\right| \widetilde{\boldsymbol{\zeta}}_{t} \hat{e}_{t, \tau}\right) \stackrel{D}{\longrightarrow} \chi_{(q)}^{2},
$$


em que $\widetilde{\boldsymbol{\Omega}}(\tau)$ tem ordem $q$, e é estimado da mesma forma que $\hat{\boldsymbol{\Omega}}(\tau)$, substituindo $\boldsymbol{\zeta}_{t}$ por $\widetilde{\boldsymbol{\zeta}}_{t}$.

Testar $H_{0}$ contra $H_{1}$ significa testar se existe correlação entre os resíduos do modelo sob $H_{0}$ e as variáveis do modelo sob $H_{1}$, calculando a esperança dada em (5.24), que resulta numa estatística seguindo uma distribuição Qui-Quadrado. Sendo assim, a um nível de significância pré-fixado para o teste, após cálcular o P-valor, e determinar a Região Crítica é possível deicidir se $H_{0}$ é rejeitada ou não, considerando sob o modelo alternativo o fato testar se a estatística é maior que zero.

Os modelos CARE introduzidos e detalhados neste capítulo são medidas de risco, que, assim como nos modelos CAViaR, visam fornecer medidas que possam auxiliar no gerenciamento dos riscos de mercado, aos quais as empresas estão expostas, fornecendo modelos que imcorporam o impacto que a magnitude dos dados causam a essas medidas. 


\section{Capítulo 6}

\section{ES - Expected Shortfall}

\subsection{Conceitos}

O VaR, como comentado e definido anteriormente é uma medida de risco muito utilizada, mas que apresenta algumas fragilidades, como por exemplo não ser uma medida de risco coerente e não apresentar informações sobre os retornos que estão acima ou abaixo do Valor em Risco, dependendo da posição.

Assim, considerando uma posição vendida, de acordo com Artzner, Delbaen, Eber e Heath (1999), e McNeil, Frey e Embrechts (2005), Expected Shortfall (ES) é uma medida de risco definida como a esperança condicional dos retornos, dado que excedem o valor do VaR. Já para posições compradas, ES é a esperança condicional dos retornos que estão abaixo do valor do VaR estimado. Dessa forma, considerando $L$ a v.a. que representa a variação percentual de preço e fixando $\theta$ para uma posição vendida, estima-se $\operatorname{VaR}(\theta)$ definido anteriormente e calcula-se

$$
\widehat{E S}_{L}(\theta)=E\left\{L \mid L \geq \widehat{\operatorname{VaR}}_{L}(\theta)\right\}
$$

assim, o VaR é a perda máxima que pode ser excedida só em $(1-\theta) \%$ dos casos e $E S_{L}(\theta)$ é definida como uma combinação convexa de $\operatorname{Va} R_{L}(\theta)$ e a esperança condi- 
cional das perdas que excedem o $\operatorname{Va} R_{L}(\theta)$, em que ES é dado por:

$$
E S_{L}(\theta)=\frac{1}{1-\theta} \int_{\theta}^{1} \operatorname{VaR}_{L}(\theta) d \theta
$$

Para posição comprada,

$$
\operatorname{ES}_{L}(\theta)=\frac{1}{\theta} \int_{0}^{\theta} \operatorname{VaR}_{L}(\theta) d \theta
$$

Para mostrar que $E S(\theta)$ é uma medida de risco coerente, basta provar que ela satisfaz os quatro axiomas definidos no Apêndice B.

De fato, os axiomas translação invariante, positividade homogênea e monotonocidade, são verificados pelas propriedades dos quantis. Logo, é necessário mostrar que a medida de risco é subaditiva.

Sendo assim, de acordo com Acerbi e Tasche (2002), considerando as variáveis aleatórias $X$ e $Y$, com $E\{X\} \leq \infty$ e $E\{Y\} \leq \infty$, e escrevendo (6.2) como

$$
E S_{L}(\theta)=\frac{1}{\theta} E\left[L I_{\left(L \leq l_{\theta}\right)}\right]
$$

em que $I$ é a função indicadora em $L$ e $l_{\theta}$ o $\theta$-quantil de $L$, deve-se provar que

$$
E S_{X+Y}(\theta) \leq E S_{X}(\theta)+E S_{Y}(\theta)
$$

Considere $Z=X+Y$. Faz-se então, de acordo com (6.3),

$$
\begin{aligned}
E S_{X}(\theta)+E S_{Y}(\theta)-E S_{Z}(\theta) & =\frac{1}{\theta} E\left[X I_{\left(X \leq x_{\theta}\right)}+Y I_{\left(Y \leq y_{\theta}\right)}-Z I_{\left(Z \leq z_{\theta}\right)}\right] \\
& =\frac{1}{\theta} E\left[X I_{\left(X \leq x_{\theta}\right)}+Y I_{\left(Y \leq y_{\theta}\right)}-(X+Y) I_{\left(Z \leq z_{\theta}\right)}\right] \\
& =\frac{1}{\theta} E\left[X\left(I_{\left(X \leq x_{\theta}\right)}-I_{\left(Z \leq z_{\theta}\right)}\right)+Y\left(I_{\left(Y \leq y_{\theta}\right)}-I_{\left(Z \leq z_{\theta}\right)}\right)\right] \\
& \geq x_{\theta} E\left[\left(I_{\left(X \leq x_{\theta}\right)}-I_{\left(Z \leq z_{\theta}\right)}\right)\right]+y_{\theta} E\left[\left(I_{\left(Y \leq y_{\theta}\right)}-I_{\left(Z \leq z_{\theta}\right)}\right)\right] \\
& =x_{\theta}(\theta-\theta)+y_{\theta}(\theta-\theta)=0,
\end{aligned}
$$

daí, segue que

$$
E S_{X}(\theta)+E S_{Y}(\theta) \geq E S_{X+Y}(\theta)
$$


o que mostra que esta é uma medida de risco coerente.

Para o cálculo desta medida de risco, uma maneira simples de calculá-la é através da estimação do $V a R$ pela função empírica, e depois a média simples dos retornos que excedem o valor do $V a R$. Mas em alguns casos, dependendo do comportamento dos retornos, é possível supor, que dadas as informações passadas, os retornos podem seguir algumas distribuições, como as apresentadas a seguir.

\subsection{ES Usando a Distribuição Normal}

Assim como no caso do $V a R$, considera-se o modelo

$$
r_{t}=\mu_{t}+\sigma_{t} \epsilon_{t}
$$

onde $\mu_{t}$ e $\sigma_{t}$ variam no tempo e seguem as estruturas dos modelos ARMA(p,q)$\operatorname{GARCH}(\mathrm{r}, \mathrm{s})$.

Como visto anteriormente, supondo que $\epsilon_{t} \sim N(0,1)$,

$$
r_{t} \mid \mathcal{F}_{t-1} \sim N\left(\mu_{t}, \sigma_{t}^{2}\right) .
$$

De acordo com a definição de $E S$, deseja-se obter, para uma posição vendida

$$
\widehat{E S}_{t}(\theta)=E\left\{\left(r_{t} \mid \mathcal{F}_{t-1}\right) \mid\left(r_{t} \mid \mathcal{F}_{t-1}\right) \geq \widehat{\operatorname{VaR}}_{t}(\theta)\right\} .
$$

Mas, note que (6.5) é, na verdade a média de uma nova variável aleatória denominada normal truncada em um intervalo $[\operatorname{VaR}(\theta),+\infty)$.

De fato, segundo McNeil, Frey e Embrechts (2005), considere uma v.a. $X \sim N\left(\mu, \sigma^{2}\right)$. Dado um intervalo $(A, B), X \in(A, B)$ é uma nova v.a., tal que

$$
E(X)=\mu+\frac{\sigma\left(\phi_{1}-\phi_{2}\right)}{T},
$$

em que

$$
T=\Phi\left(\frac{B-\mu}{\sigma}\right)-\Phi\left(\frac{A-\mu}{\sigma}\right), \phi_{1}=f\left(\frac{A-\mu}{\sigma}\right) \text { e } \phi_{2}=f\left(\frac{B-\mu}{\sigma}\right),
$$


sendo $f($.$) a função densidade de probabilidade e \Phi($.$) a função de probabilidade$ acumulada de uma distribuição normal padrão.

Sabendo que

$$
L=\frac{r_{t} \mid \mathcal{F}_{t-1}-\mu_{t}}{\sigma_{t}} \sim N(0,1),
$$

e considerando as propriedades do quantil de uma v.a.,

$$
\begin{aligned}
E S_{\{L\}}(\theta) & =\frac{E S_{\left\{r_{t} \mid \mathcal{F}_{t-1}\right\}}(\theta)-\mu_{t}}{\sigma_{t}} \Rightarrow \\
E S_{\left\{r_{t} \mid \mathcal{F}_{t-1}\right\}}(\theta) & =\mu_{t}+\sigma_{t} E S_{\{L\}}(\theta) .
\end{aligned}
$$

Assim,

$$
\widehat{E S}_{\left\{r_{t} \mid \mathcal{F}_{t-1\}}\right.}(\theta)=\hat{\mu}_{t}+\hat{\sigma}_{t} \widehat{E S}_{\{L\}}(\theta) .
$$

Mas, para $(A, B)=\left(\operatorname{VaR}_{\{L\}}(\theta) \leq L \leq+\infty\right)$,

$$
\widehat{E S}_{\{L\}}(\theta)=\frac{\phi\left(\Phi^{-1}(\theta)\right)}{1-\theta},
$$

onde $\phi($.$) é a função densidade de probabilidade de L$, ou seja, a f.d.p. da normal padrão.

Logo,

$$
\widehat{V a R}_{\{L\}}(\theta)=\Phi^{-1}(\theta) \text { e } T=\Phi(B)-\Phi\left(\Phi^{-1}(\theta)\right)=1-\theta,
$$

onde $\Phi(B)=1, \phi_{1}=\phi\left(\Phi^{-1}(\theta)\right)$ e $\phi_{2}=0$, quando $B \rightarrow+\infty$.

Assim, substituindo (6.7) em (6.6), para $r_{t} \mid \mathcal{F}_{t-1}$,

$$
\begin{aligned}
E S_{t}(\theta) & =\mu_{t}+\sigma_{t}\left[\frac{\phi\left(\Phi^{-1}(\theta)\right)}{1-\theta}\right] \Rightarrow \\
\widehat{E S}_{t}(\theta)[h] & =\hat{\mu}_{t}(h)+\hat{\sigma}_{t}(h)\left[\frac{\phi\left(\Phi^{-1}(\theta)\right)}{1-\theta}\right] .
\end{aligned}
$$




\subsection{ES Usando a Distribuição t-Student}

De acordo com o comportamento dos retornos, no caso de apresentarem uma cauda mais pesada, pode se supor distribuição t-Student aos dados. Assim, de acordo com o modelo (6.4), supondo $\epsilon_{t} \sim t(0,1, v)$,

$$
r_{t} \mid \mathcal{F}_{t-1} \sim t\left(\mu_{t}, \sigma_{t}^{2}, v\right) .
$$

Fazendo

$$
L=\frac{r_{t} \mid \mathcal{F}_{t-1}-\mu_{t}}{\sigma_{t}} \sim t(0,1, v),
$$

e como no caso da distribuição Normal,

$$
\widehat{E S}_{\left\{r_{t} \mid \mathcal{F}_{t-1}\right\}}(\theta)=\hat{\mu}_{t}+\widehat{E S}_{\{L\}}(\theta) \hat{\sigma}_{t} .
$$

Sendo assim,

$$
\begin{aligned}
E S_{\{L\}}(\theta) & =E\left\{L \mid L \geq \operatorname{VaR}_{L}(\theta)\right\} \\
\Rightarrow E S_{\{L\}}(\theta) & =\frac{1}{1-\theta} \int_{t^{-1}(\theta)}^{+\infty} l f(l) d l,
\end{aligned}
$$

em que $f($.$) é a f.d.p. de l$ e $t^{-1}(\theta)$ a inversa da função de distribuição, dada em (6.8). De fato, seja

$$
\frac{1}{1-\theta} \int_{t^{-1}(\theta)}^{+\infty} l f(l) d l=\frac{1}{1-\theta} \int_{t^{-1}(\theta)}^{+\infty} l \frac{\Gamma\left(\frac{v+1}{2}\right)}{\sqrt{v \pi} \Gamma\left(\frac{v}{2}\right)}\left(1+\frac{l^{2}}{v}\right)^{-\left(\frac{v+1}{2}\right)} d l .
$$

Fazendo

$$
\begin{aligned}
k & =\frac{\Gamma\left(\frac{v+1}{2}\right)}{\sqrt{v \pi} \Gamma\left(\frac{v}{2}\right)} \\
\mathrm{e} 1+\frac{l^{2}}{v} & =u \Rightarrow \frac{v}{2} d u=l d l
\end{aligned}
$$


de acordo com (6.10),

$$
\begin{aligned}
E S_{\{L\}}(\theta) & =\frac{k}{1-\theta}\left[\int_{\frac{\left\{t^{-1}(\theta)\right\}^{2}}{v}+1}^{+\infty} u^{-\left(\frac{v+1}{2}\right)} d u \frac{v}{2}\right] \\
& =\frac{k v}{2(1-\theta)}\left[-\left(\frac{\left\{t^{-1}(\theta)\right\}^{2}}{v}+1\right)^{-\left(\frac{v+1}{2}\right)+1}\left(\frac{2}{-v+1}\right)\right] \\
& =\frac{k v}{2(1-\theta)}\left(\frac{2}{-v+1}\right)\left[-\left(\frac{\left\{t^{-1}(\theta)\right\}^{2}}{v}+1\right)^{-\left(\frac{v+1}{2}\right)+1}\right] \\
& =\frac{k}{(1-\theta)}\left(\frac{v}{v-1}\right)\left[\left(\frac{\left\{t^{-1}(\theta)\right\}^{2}}{v}+1\right)^{-\left(\frac{v+1}{2}\right)}\right]\left[\frac{\left\{t^{-1}(\theta)\right\}^{2}}{v}+1\right] .
\end{aligned}
$$

Fazendo

$$
\begin{array}{r}
f\left(t^{-1}(\theta)\right)=k\left[\left(\frac{\left\{t^{-1}(\theta)\right\}^{2}}{v}+1\right)^{-\left(\frac{v+1}{2}\right)}\right], \text { tem-se } \\
E_{\{L\}}(\theta)=\frac{f\left(t^{-1}(\theta)\right)}{(1-\theta)}\left[\frac{v+\left\{t^{-1}(\theta)\right\}^{2}}{v-1}\right] .
\end{array}
$$

Assim, de acordo com (6.9), para $r_{t} \mid \mathcal{F}_{t-1}$,

$$
\begin{aligned}
E S_{t}(\theta) & =\mu_{t}+\sigma_{t}\left[\left(\frac{f\left(t^{-1}(\theta)\right)}{1-\theta}\right)\left(\frac{v+\left\{t^{-1}(\theta)\right\}^{2}}{v-1}\right)\right] \Rightarrow \\
\widehat{E S}_{t}(\theta)[h] & =\hat{\mu}_{t}(h)+\hat{\sigma}_{t}(h)\left[\left(\frac{f\left(t^{-1}(\theta)\right)}{1-\theta}\right)\left(\frac{v+\left\{t^{-1}(\theta)\right\}^{2}}{v-1}\right)\right] .
\end{aligned}
$$

\subsection{ES Utilizando Expectil}

Um outro método, proposto por Taylor (2008), é o uso do $\tau$ - expectil como um estimador para o $\theta$ - quantil, possibilitando uma nova alternativa para a estimação de ES. Considere $M Q A$ dado por

$$
M Q A=\min _{m \in \mathbb{R}}\left[E\left\{\rho_{\tau}(Y-m)^{2}\right\}\right]
$$


para um $\tau$ fixo, como visto anteriormente em (5.4)

$$
\begin{aligned}
\left(\frac{1-2 \tau}{\tau}\right) \int_{-\infty}^{\mu(\tau)}(Y-\mu(\tau)) d F_{Y}(y) & =\mu(\tau)-E(Y) \\
\Rightarrow E\left\{(Y-\mu(\tau)) I_{(y \leq \mu(\tau))}\right\} & =\left(\frac{\tau}{1-2 \tau}\right)[\mu(\tau)-E(Y)] \\
\Rightarrow E\left\{Y I_{(y \leq \mu(\tau))}\right\}-E\left\{\mu(\tau) I_{(y \leq \mu(\tau))}\right\} & =\left(\frac{\tau}{1-2 \tau}\right)[\mu(\tau)-E(Y)] \\
E\left\{Y I_{(y \leq \mu(\tau))}\right\}-\mu(\tau) E\left\{I_{(y \leq \mu(\tau))}\right\} & =\left(\frac{\tau}{1-2 \tau}\right)[\mu(\tau)-E(Y)] .
\end{aligned}
$$

Utilizando $\mu(\tau)$ como estimador para o $\theta$ - quantil, tal que $F(\mu(\tau))=\theta$, onde $F($.$) é a f.d. de Y, tem-se:$

$$
E\left\{I_{(y \leq \mu(\tau))}\right\}=\theta .
$$

Substituindo (6.12) em (6.11), tem:

$$
E\left\{Y I_{(y \leq \mu(\tau))}\right\}-\mu(\tau)(\theta)=\left(\frac{\tau}{1-2 \tau}\right)[\mu(\tau)-E(Y)] .
$$

Dividindo ambos os lados da igualdade por $\theta$, tem-se:

$$
\begin{array}{r}
\frac{E\left\{Y I_{(y \leq \mu(\tau))}\right\}-\mu(\tau)(\theta)}{\theta}=\left(\frac{\tau}{1-2 \tau}\right) \frac{\mu(\tau)}{\theta}-\left(\frac{\tau}{1-2 \tau}\right) \frac{E(Y)}{\theta} \\
\Rightarrow \frac{E\left\{Y I_{(y \leq \mu(\tau))}\right\}}{\theta}=\left(1+\frac{\tau}{(1-2 \tau) \theta}\right) \mu(\tau)-\frac{\tau}{(1-2 \tau) \theta} E(Y) \\
\Rightarrow E S(\theta)=\left(1+\frac{\tau}{(1-2 \tau) \theta}\right) \mu(\tau)-\frac{\tau}{(1-2 \tau) \theta} E(Y) .
\end{array}
$$

Estendendo a estimação de $E S$ para uma sequência $r_{t}$, com $t=(1, \ldots, T)$, tem-se:

$$
\widehat{E S}_{t}(\theta)=\left(1+\frac{\tau}{(1-2 \tau) \theta}\right) \hat{\mu}_{t}(\tau)-\frac{\tau}{(1-2 \tau) \theta} E\left(r_{t} \mid \mathcal{F}_{t-1}\right) .
$$

Note que a estimativa do $E S$ pelo expectil, é resultado da ligação entre a minimização da função de perda e a definição de $E S$, utlizando o $\tau$ - expectil como estimativa para o $\theta-q u a n t i l$. 
Sendo assim, de acordo com (6.13), pode-se ver que para o modelo condicional, o valor do $\tau$-expectil é proporcional ao valor de $E S$.

Utilizando as estruturas do modelo CARE, pode se estimar vários modelos autorregressivos para o $E S$, condicional a informações passadas. Por exemplo o modelo CARE - Absoluto Simétrico definido em (5.9), tal que

$$
\mu_{t}(\tau)=\beta_{0}+\beta_{1} \mu_{t-1}(\tau)+\beta_{2}\left|r_{t-1}\right|,
$$

em um modelo onde $E\left(r_{t} \mid \mathcal{F}_{t-1}\right)=0$, substituindo (6.14) em (6.13),

$$
E S_{t}(\theta)=\gamma_{0}+\gamma_{1} E S_{t-1}(\theta)+\gamma_{2}\left|r_{t-1}\right|,
$$

em que $\gamma_{1}=\beta_{1}$, e para $j=0$ e $2, \gamma_{j}=\left(1+\frac{\tau}{(1-2 \tau) \theta}\right) \beta_{j}$.

Utilizando as outras especificações, utilizando a mesma idéia é possível estimar o ES.

\subsection{ES Usando Teoria dos Valores Extremos}

Assim como na modelagem do VaR, ES também pode ser estimado utilizando a TVE por dois métodos, os quais são chamados, no trabalho de ES(TVE1) e ES(TVE2).

\subsubsection{ES(TVE1)}

De fato, ao considerar a distribuição de uma v.a. que excede um limiar $u$, para o cálculo do ES, de acordo com a efinição de ES, deseja-se obter a média dessa nova v.a. que está acima do VaR(TVE1), estimado, ou seja, sabendo que

$$
\widehat{V a R}_{t}(T V E 1)(\theta)=u+\frac{\hat{\beta}_{t, u}}{\hat{\xi}_{t}}\left[\left(\frac{1-\theta}{\overline{F_{n}(u)}}\right)^{-\hat{\xi}_{t}}-1\right],
$$

considerando $X$ uma nova v.a., tem-se:

$$
E S(T V E 1)(\theta)=E\{X \mid X \geq \widehat{V a R}(T V E 1)(\theta)\} .
$$


Considerando que foi ajustada uma DGP aos retornos que excedem o limiar $u$ até o instante $t-1$ e tomou-se $\widehat{V a R}_{t}(T V E 1)(\theta)$ como o $\theta$ - quantil dessa distribuição, ao considerar a mesma série de log-retornos, $\widehat{E S}_{t}(T V E 1)(\theta)$ seria a média de uma nova DGP, em que $V a R(T V E 1)>u$ seria um novo limiar.

Sendo assim, utilizando as propriedades da média dos excessos, vistas no Capítulo 2, tem-se:

$$
\begin{aligned}
\widehat{E S}_{t}(T V E 1)(\theta) & =\widehat{V a R}_{t}(T V E 1)(\theta)+E\left\{X-\widehat{V a R}_{t}(T V E 1)(\theta) \mid X \geq \widehat{V a R}(T V E 1)(\theta)\right\} \\
& =\widehat{V a R}_{t}(T V E 1)(\theta)+\frac{\hat{\beta}_{t, u}+\hat{\xi}\left(\widehat{V a R}_{t}(T V E 1)(\theta)-u\right)}{1-\xi} \\
& =\frac{\widehat{V a R}_{t}(T V E 1)(\theta)}{1-\hat{\xi}}+\frac{\hat{\beta}_{t, u}-\hat{\xi} u}{1-\hat{\xi}} .
\end{aligned}
$$

\subsubsection{ES(TVE2)}

Assim como VaR(TVE2), considere uma série de retornos $r_{t}$, sob o modelo $r_{t}=\mu_{t}+$ $\sigma_{t} \epsilon_{t}$. Depois de ajustar um modelo $\operatorname{ARMA}(\mathrm{p}, \mathrm{q})-\operatorname{GARCH}(\mathrm{r}, \mathrm{s})$, calcula-se o resíduos padronizados, e aos résíduos que excedem o limiar $u$ apropriado, ajusta-se uma DGP obtendo-se $\hat{\beta}_{u}$ e $\hat{\xi}$.

Neste contexto,

$$
E S_{t}(T V E 2)(\theta)=\mu_{t}+\sigma_{t} E\left\{Z \mid Z \geq q_{D G P}(\theta)\right\},
$$

em que $Z$ é a série de resíduos padronizados.

Mas,

$$
E\left\{Z \mid Z \geq q_{D G P}(\theta)\right\}=\frac{q_{D G P}(\theta)}{1-\hat{\xi}}+\frac{\hat{\beta}_{u}-\hat{\xi} u}{1-\hat{\xi}} .
$$

Sendo assim,

$$
\begin{aligned}
E S_{t}(T V E 2)(\theta) & =\mu_{t}+\sigma_{t}\left(\frac{q_{D G P}(\theta)}{1-\hat{\xi}}+\frac{\hat{\beta}_{u}-\hat{\xi} u}{1-\hat{\xi}}\right) \Rightarrow \\
\widehat{E S}_{t}(T V E 2)(\theta)[h] & =\hat{\mu}_{t}(h)+\hat{\sigma}_{t}(h)\left(\frac{q_{D G P}(\theta)}{1-\hat{\xi}}+\frac{\hat{\beta}_{u}-\hat{\xi} u}{1-\hat{\xi}}\right) .
\end{aligned}
$$


É possível, em moldes similares, desenvolver testes capazes de verificar se os modelos de ES são adequados ou não, assim como nos modelos de VaR.

\subsection{Verificação dos Métodos e Modelos para o ES}

A idéia principal, de acordo com McNeil e Frey (2000), é obter um teste que não faça suposições sobre a distribuição dos excessos, e nem sobre os resíduos dos modelos ajustados .

Inicialmente, o teste considera somente os retornos que excedem o valor do quantil condicional ajustado, o VaR.

Considere todos os retornos $r_{t}$ tal que

$$
r_{t}>\operatorname{Va} R_{t}(\theta)
$$

para posição vendida e

$$
r_{t}<\operatorname{Va} R_{t}(\theta)
$$

para posição comprada.

Após o ajuste do ES para cada instante de tempo, temos os excessos com as seguintes estruturas

$$
Z_{t}=\frac{r_{t}-E S_{t}(\theta)}{\sigma_{t}}
$$

onde $Z_{t}$ são variáveis aleatórias i.i.d..

Note que para os modelos CARE propostos por Taylor (2008) e para os métodos utilizando a TVE, onde a volatilidade não é estimada para o cálculo do ES, o teste bootstrap é adaptado. Ao ínvés de padronizar os excessos pela volatilidade condicional estimada, os excessos são padronizados pelo quantil condicional estimado, ou seja:

$$
Z_{t}=\frac{r_{t}-E S_{t}(\theta)}{\operatorname{VaR}_{t}(\theta)}
$$


Para esses excessos, sob o a hipótese nula de que o modelo ou método estima corretamente o ES, espera-se que tenham média zero, e para isso, constrói-se o teste bootstrap $^{1}$, em que deseja-se testar, para posição comprada,

$$
\left\{\begin{array}{l}
H_{0}: \mu_{Z}=0 \\
H_{1}: \mu_{Z}<0
\end{array}\right.
$$

que é equivalente a testar se o modelo ou modelo sistematicamente, superestima ou não o ES.

\footnotetext{
${ }^{1}$ Para mais detalhes, veja o Apêndice D.
} 


\section{Capítulo 7}

\section{Aplicações}

Neste capítulo, serão apresentados os cálculos das medidas de risco definidas anteriormente, visando observar como elas se comportam de acordo com o cenário.

Considerou-se, para a aplicação o Índice da Bolsa de Valores de São Paulo (IBOVESPA).

\subsection{Descrição da Série}

O Ibovespa é o mais importante indicador do desempenho médio das cotações das ações negociadas na Bolsa de Valores de São Paulo. É formado pelas ações com maior volume negociado. O índice é calculado em tempo real, considerando instantaneamente os preços de todos os negócios efetuados no mercado à vista com as ações componentes de sua carteira. A série utilizada para o cálculo das medidas de risco é constituída de valores no período de 04/07/1994 a 11/02/2010, totalizando 3.862 observações.

A Figura 7.1 apresenta o gráfico da série e dos log-retornos, respectivamente.

A análise da Figura 7.1 revela que o IBOVESPA, assim como outros indicadores, sofreu impacto da crise econômica de 2008 a 2009, causada pela quebra de um dos maiores bancos de investimentos do mundo, o Lehman Brothers e, apesar de orgãos do governo brasileiro afirmarem que a crise não afetaria o país, o índice da Bolsa de 



Figura 7.1: Gráficos da série de IBOVESPA e da série de log-retornos.

São Paulo mostrou como o período foi de grande instabilidade.

A Figura 7.2 apresenta o histograma e o QQ-Plot dos log-retornos do IBOVESPA, que podem auxiliar na análise do comportamento da série.
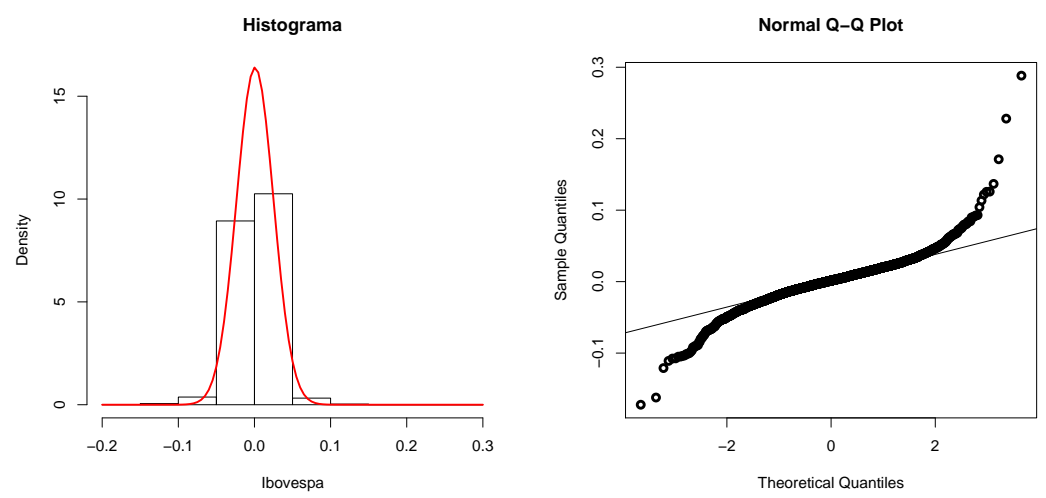

Figura 7.2: Gráficos do histograma com densidade normal ajustada e QQ-Plot dos log-retornos.

De acordo com o histograma, a série parece apresentar um comportamento assimétrico, e pelo QQ-Plot, há indícios de que a série não seja normalmente distribuída.

A Tabela 7.1 apresenta as estatísticas descritivas, pelas quais pode-se observar 


\begin{tabular}{c|c}
\hline Média & 0,000844 \\
\hline Mediana & 0,001433 \\
\hline Desvio Padrão & 0,02392736 \\
\hline Assimetria & 0,458397 \\
\hline Curtose & 15,61384 \\
\hline $1^{\underline{o}}$ Quartil & $-0,011120$ \\
\hline $3^{\text {o }}$ Quartil & 0,0138 \\
\hline Mínimo & $-0,1723$ \\
\hline Máximo & 0,2882 \\
\hline
\end{tabular}

Tabela 7.1: Estatísticas da série de log-retornos do IBOVESPA.

que os dados apresentam curtose alta, indicando que a série tem uma distribuição com caudas pesadas. Já o coeficiente de assimetria indica que a série apresenta uma leve assimetria, com média em torno de zero.

Para o cálculo das medidas do Valor em Risco, de acordo como foram definidas e comentadas nos capítulos anteriores, primeiramente utiliza-se a abordagem econométrica, depois TVE, os modelos CAViaR e CARE. Calcula-se também o ES, uma medida de risco coerente, visando analisar e discutir como se comportam os retornos que estão acima ou abaixo do valor do VaR, de acordo com a posição desejada.

O cálculo das estimativas, tanto nos modelos com abordagem econométrica, como nos modelos CaViaR, CARE e ES, foram feitos utilizando os primeiros 3.361 logretornos, no período de 04/07/1994 a 31/01/2008, deixando os últimos 500 logretornos, no período de 01/02/2008 a 11/02/2010, para fins de testes pós-amostrais, com o objetivo de verificar se o modelo utilizado é ou não adequado. 


\subsection{Cálculo do VaR}

\subsubsection{Abordagem Econométrica}

Considerando as estruturas dos modelos $\operatorname{ARMA}(\mathrm{p}, \mathrm{q})-\mathrm{GARCH}(\mathrm{r}, \mathrm{s})$, foram ajustados modelos supondo erros com distribuição Normal, T-Student e T-generalizada assimétrica, mas pelo que foi notado nos ajustes, pela aplicação do teste Ljung-Box aos resíduos padronizados e aos quadrados dos resíduos padronizados, verificou-se que os resíduos ainda eram correlacionados e apresentavam heteroscedasticidade condicional. Sendo assim, foi feito um estudo que revelou que o melhor modelo para a série de log-retornos do IBOVESPA é um AR(10)-TARCH(1,1), que é um modelo assimétrico que visa incorporar o efeito que as informações podem causar na volatilidade, modelo este detalhado no Apêndice $\mathrm{E}, \operatorname{com} \epsilon_{t} \sim t_{9,58}$, dado por:

$$
\begin{aligned}
r_{t} & =0,0014+0,029 r_{t-1}-0,04 r_{t-4}-0,0342 r_{t-5}+0,04 r_{t-10}+a_{t} \\
a_{t} & =\sigma_{t} \epsilon_{t}, \\
\sigma_{t}^{2} & =0,00001759+0,026 a_{t-1}^{2}+0,14 S_{t-1} a_{t-1}^{2}+0,86 \sigma_{t-1}^{2} .
\end{aligned}
$$

Para o ajuste do modelo foi utilizado o software $S+$ Finmetrics.

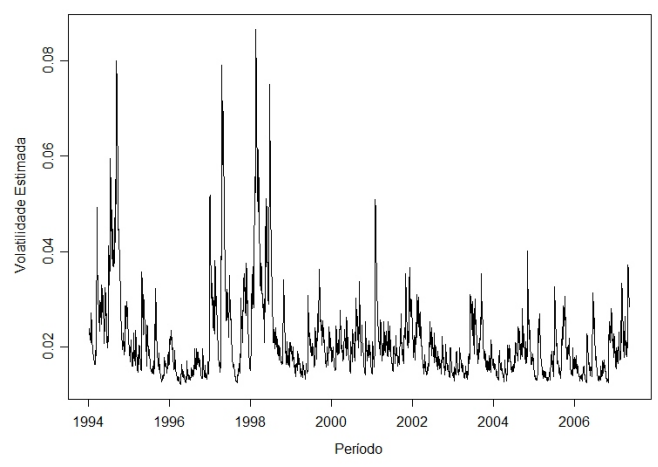

Figura 7.3: Estimativa da volatilidade para os retornos do IBOVESPA.

A Figura 7.3 apresenta a volatilidade estimada da série de log-retornos do IBOVESPA 
utilizando o modelo (7.1). Os picos observados são referentes a março de 1995, durante a crise do México, outubro de 1997, período final da crise da Ásia, início de setembro de 1996, a desvalorização do Real, em junho de 2003 devido a relação entre o mercado brasileiro e asiático e em julho de 2005, devido aos investimentos de empresas visando sustentabilidade.

A Figura 7.4 apresenta o QQ-Plot dos Resíduos Padronizados, comparando os quantis dos mesmos com os quantis da distribuição teórica, no caso t-Student.

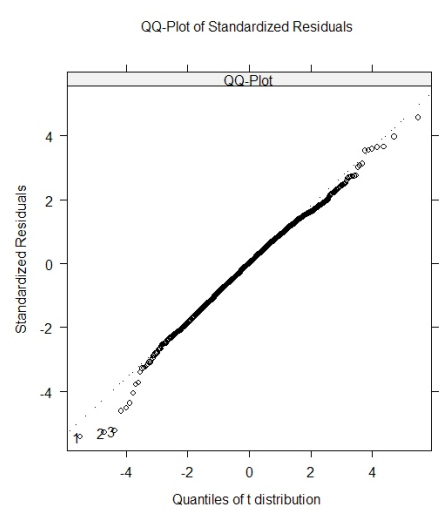

Figura 7.4: QQ-Plot dos resíduos padronizados.

O gráfico indica que, aparentemente, os resíduos seguem uma distribuição $t_{9,58}$.

Com o objetivo de testar se os resíduos padronizados realmente seguem uma distribuição $t_{9,58}$, faz-se o teste de Kolmogorov Smirnov; a Tabela 7.2 fornece os valores da estatística e do P-valor do teste, que a um nível de significância de $5 \%$ não rejeita a hipótese de que os resíduos padronizados realmente seguem a distribuição teórica proposta.

Para o cálculo do VaR, inicialmente é necessário definir quais posições estão em estudo.

Ao fazer o cálculo do VaR para posição comprada, o interesse é calcular com dada probabilidade $\theta$, o máximo que um investidor, sob condições normais de mercado, poderá perder se investir uma dada quantia. Assim, o investidor espera que a proba- 


\begin{tabular}{c||c}
\hline \multicolumn{2}{c}{ Teste Kolmogorov-Smirnov } \\
\hline \hline Estatística do Teste & 0,016 \\
\hline P-valor & 0,75 \\
\hline
\end{tabular}

Tabela 7.2: Valores do Teste de Kolmogorov-Smirnov

bilidade de perda, de acordo com a variação do valor do ativo seja pequena. Por isso, o valor adotado para a probabilidade é $\theta=0,05$.

No caso de posição vendida, na visão das empresas que alugam um ativo em data futura, espera-se que a queda do valor do ativo, ocorra com uma probabilidade alta, na maioria das vezes, o valor adotado é $\theta=0,95$.

Assim, para um $\theta=0,05$, calcula-se o VaR. Para o ajuste, fez-se a suposição de que $\epsilon_{t} \sim t_{9,58}$.

Sendo assim, utilizando o modelo (7.1), encontrou-se as previsões da média e da volatilidade um passo à frente, que resultam em:

$$
\begin{aligned}
& \hat{r}_{3361}(1)=0,001187165 \\
& \hat{\sigma}_{3361}^{2}(1)=0,00066761
\end{aligned}
$$

e utilizando o 0,05 - quantil da distribuição t-Student com 9, 58 g.l.,

$$
q_{t_{9,581578}^{*}}^{*}(0,05)=-1,820526
$$

tem-se que

$$
\begin{aligned}
& \widehat{\operatorname{VaR}}_{3361}(0,05)[1]=0,001187165-1,820526(0,00066761)^{\frac{1}{2}} \Rightarrow \\
& \widehat{\operatorname{VaR}}_{3361}(0,05)[1]=-0,04065553 .
\end{aligned}
$$

Isto significa que, sob condições normais de mercado, com probabilidade de 5\%, o máximo que um investidor poderá perder em um período de 1 dia será, aproximadamente $4,06 \%$ do valor investido na carteira, de acordo com essa abordagem. 
A seguir, utilizando a série juntamente com as 500 observações restantes faz-se, de acordo com o modelo (7.1), o gráfico das estimativas do VaR para posições compradas e vendidas.

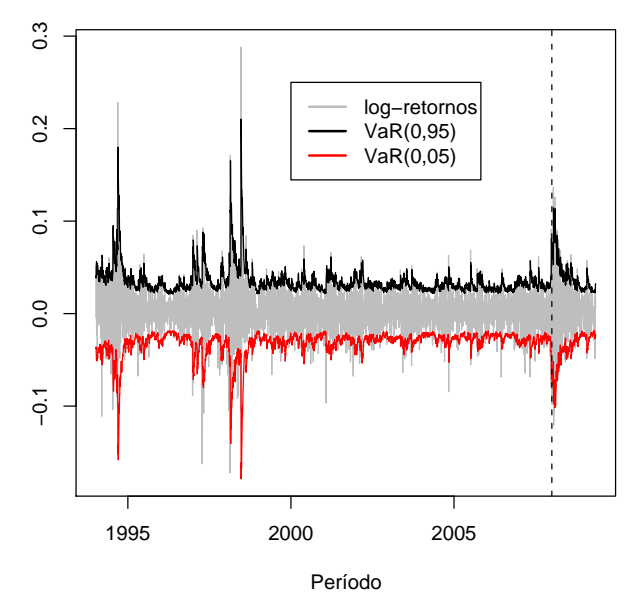

Figura 7.5: Estimativas para o VaR, $\operatorname{com} \theta=0,05$ e $\theta=0,95$.

Ao analisar o gráfico, é possível notar que os valores do VaR são ultrapassados por alguns valores dos log-retornos, totalizando 269 excessos.

\subsubsection{Teoria dos Valores Extremos}

Inicialmente, antes de estimar qualquer medida de risco, é necessário que se escolha o limiar apropriado.

A Figura 7.6 mostra a média dos excessos versus os valores do limiar para a série de log-retornos, no qual o valor mais adequado é aquele qua apresenta a menor média. De acordo com essa figura, escolheu-se como limiar, o valor $u=0$.

Para os ajustes do VaR(TVE1), utilizou-se, como estimativa para o $V a R_{t}(\theta)(T V E 1)$ os primeiros $60 \log$-retornos, em que $t=60$, assim, para cada log-retorno $r_{t+1}$, ajustase uma nova Distribuição Generalizada de Pareto, e toma-se o seu quantil como 


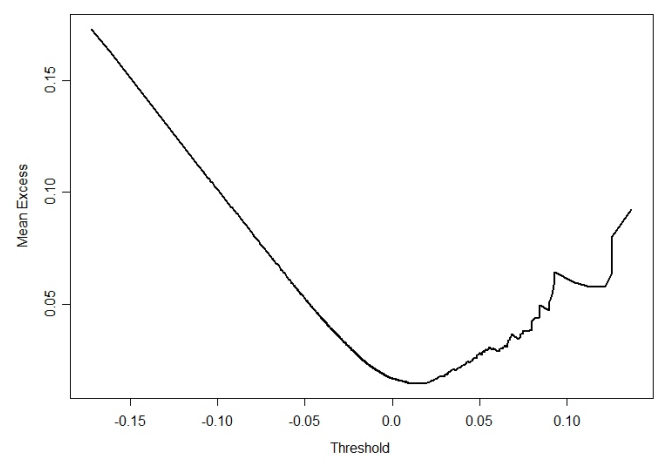

Figura 7.6: Gráfico da Média dos Excessos para os log-retornos.

$\widehat{V a R}_{t+1}(T V E 1)(\theta)$.

A Figura 7.7 apresenta os valores estimados para o VaR(TVE1). Nesta figura pode-se notar que as estimativas são bastante suaves, ou seja, apresentam pouca variablidade, podendo ser um método não muito indicado para o cálculo de medidas de risco em períodos de instabilidade, tanto para posições compradas como posições vendidas.

Finalmente, utilizando a abordagem econométrica, juntamente com Teoria de Valores Extremos, como já foi discutido no Capítulo 3, para a estimação de VaR(TVE2), faz-se a escolha do limiar.

Para isso, tem-se o gráfico da média dos excessos versus os valores do limiar para os resíduos padronizados do modelo (7.1), apresentado na Figura 7.8.

A Tabela 7.3 apresenta os valores dos limiares adotados, das estimativas dos parâmetros da DGP ajustada aos excessos dos resíduos padronizados e dos $\theta$-quantis, para $\theta=0,05$ e $\theta=0,95$.

A Figura 7.9 apresenta os valores estimados para VaR(TVE2) para $\theta=0,95$ e $\theta=0,05$.

Assim como no método utilizando abordagem econométrica, supondo distribuição $t$ - Student aos erros, os valores estimados para VaR, pelo método VaR(TVE2) 


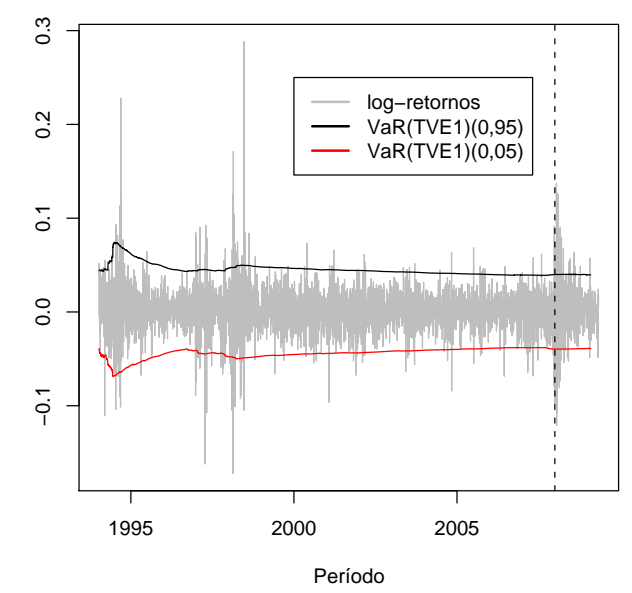

Figura 7.7: Estimativas para o VaR, $\operatorname{com} \theta=0,05$ e $\theta=0,95$ utilizando TVE.

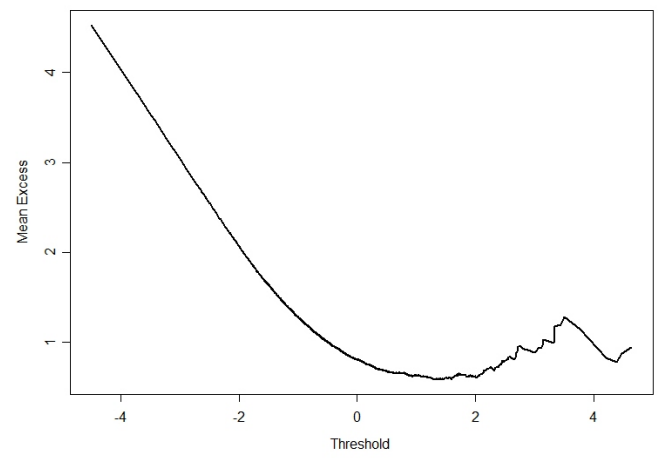

Figura 7.8: Gráfico da Média dos Excessos para os Resíduos Padronizados.

também são ultrapassados por alguns log-retornos.

Visando analisar qual o método mais adequado dentre os três utilizados, calculase, de acordo com o visto no Capítulo 3, para $\theta=0,05$ o número de excessos e suas respectivas proporções, apresentados na Tabela 7.4:

De acordo com o Capítulo 3, espera-se que se o modelo for adequado, a proporção 


\begin{tabular}{c||c|c}
\hline$\theta$ & 0,05 & 0,95 \\
\hline \hline limiar & $-1,371083$ & 1,597834 \\
\hline$\beta_{u}$ & 0,5150141 & 0,4025834 \\
\hline$\xi$ & 0,1167387 & 0,1518245 \\
\hline$q_{D G P}$ & $-1,847027$ & 1,527073 \\
\hline
\end{tabular}

Tabela 7.3: Valores Estimados para os Parâmetros das DGP ajustadas.

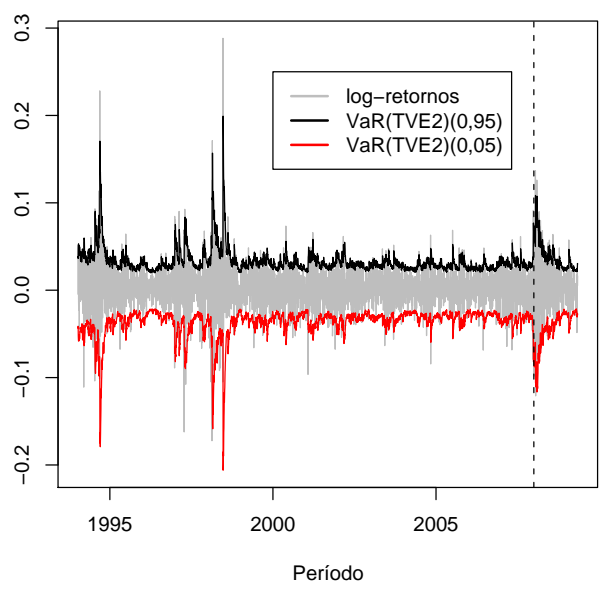

Figura 7.9: Estimativas para o VaR, $\operatorname{com} \theta=0,05$ e $\theta=0,95$ utilizando TVE.

\begin{tabular}{c||c|c|c}
\hline Método & VaR & VaR(TVE1) & VaR(TVE2) \\
\hline Excessos & 269 & 122 & 191 \\
\hline Proporção & 0,069 & 0,032 & 0,049 \\
\hline
\end{tabular}

Tabela 7.4: Número de Excessos e Proporção para os métodos adotados.

de valores que ultrapassam o VaR deve ser próxima do valor de $\theta$.

A proporção de excessos, de acordo com a Tabela 7.4, para o método considerando 
abordagem econométrica, com erros seguindo distribuição $t$-Student, apresenta valor um pouco acima do esperado, indicando que o modelo apresenta erro sistemático.

Sabendo que a distribuição do número de excessos ou violações segue uma distribuição Binomial, detalhada no Capítulo 3, é possível estabelecer a região crítica. A um nível de confiança de $95 \%$, tem-se a região crítica definida como o número de excessos $N$, tal que $N \geq 170$. Sendo assim, pela estatística do teste, dado que o número de violações atinge 269, rejeita-se a hipótese de que o modelo $\operatorname{AR}(10)-\operatorname{GARCH}(1,1)$, dado em (7.1) estima corretamente o quantil condicional para o VaR.

Da mesma forma, nota-se que o VaR(TVE1), dentre os três é o que apresenta o menor número de violações, e, consequentemente a menor proporção, indicando que o modelo estima corretamente o quantil condicional, porém, possui uma variabilidade pequena dos valores estimados, tornando-se um método não muito indicado para períodos de instabilidade.

Já o modelo VaR(TVE2) possui um número de violações que está dentro do intervalo de rejeição de $H_{0}$, porém, a proporção do números de excessos está em torno do valor esperado.

\subsubsection{Modelos CAViaR}

Para o ajuste dos modelos CAViaR, de acordo com Engle e Manganelli (2004), inicialmente gera-se $n$ vetores $\boldsymbol{\beta}$ entre 0 e 1 , em que a ordem de cada vetor depende da especificação adotada.

De acordo com a posição desejada, fixa-se o valor do $\theta$, que no trabalho, visando o cálculo das medidas de risco para posições compradas, adotou-se $\theta=0,05$.

Para cada vetor $\boldsymbol{\beta}$, aplica-se a função de Critério Quantílico (CQ) definida no Capítulo 4. Obtém-se o $m$ vetores que produziram os menores valores para o CQ.

No trabalho, foram fixados os valores $n=\left[10^{4}, 10^{5}, 10^{4}, 10^{4}, 10^{4}, 10^{4}, 10^{4}\right]$ e $m=$ $[10,15,10,5,10,10,10]$ para as 7 especificações detalhadas no Capítulo 4.

Esses $m$ vetores são utilizados como valores iniciais para o Algorítmo Quasi- 
Newton. Este procedimento é realizado até a covergência do algorítmo. O nível de tolerância para a função e os valores dos parâmetros é $10^{-10}$.

Para a estimação do modelo foi utilizado o software Matlab, com as funções de otimização fminunc e fminsearch.

Como estimativa do $\operatorname{VaR}(\theta)$ toma-se o $\theta$ - quantil das observações amostrais.

A Figura 7.10 apresenta os gráficos dos valores estimados para o VaR de acordo com as especificações do modelo.

As Tabelas 7.5 e 7.6 apresentam o valor estimado, P-valor e Erro Padrão para cada um dos parâmetros, além do valor dos $H i t_{a m}$, Hit $t_{\text {pam }}$, dos testes de $Q D_{a m}$ e $Q D_{\text {pam }}$, além do valor do $C Q$ para cada um dos modelos adotados.

As análises de todos os modelos foram feitas à um nível de significância de $5 \%$ para todos os testes.

Após a estimação, chega-se ao seguinte modelo para o $V a R$ de acordo com a especificação Valor Absoluto Simétrico:

$$
\widehat{\operatorname{VaR}}_{t}(0,05)=0,2485+0,8433 \widehat{V a R}_{t-1}(0,05)+0,2475\left|r_{t-1}\right| .
$$

Analisando os valores estimados, todos os parâmetros possuem P-valor indicando que são significativos. A proporção de Hits amostrais está em torno de 5\%, indicando que o modelo está bem ajustado. Já a proporção de Hits pós-amostrais apresenta valor de 6,8\%. O valor do teste de Quantis Dinâmicos amostral em torno de 0,07 indica que o modelo não apresenta uma boa qualidade. O teste de $Q D_{\text {pam }}$, por conter menos informações, a este de nível de significância, e por não depender do procedimento de estimação apresenta valor indicando ser um modelo razoável para o gerenciamento do risco.

A especificação Inclinação Assimétrica apresenta a seguinte modelo estimado:

$$
\begin{aligned}
\widehat{\operatorname{VaR}}_{t}(0,05)=0,2096+0,8749 \widehat{V a R}_{t-1}(0,05) & -0,0349 r_{t-1} I\left(r_{t-1}>0\right)+ \\
& +0,3268 r_{t-1} I\left(r_{t-1}<0\right) .
\end{aligned}
$$



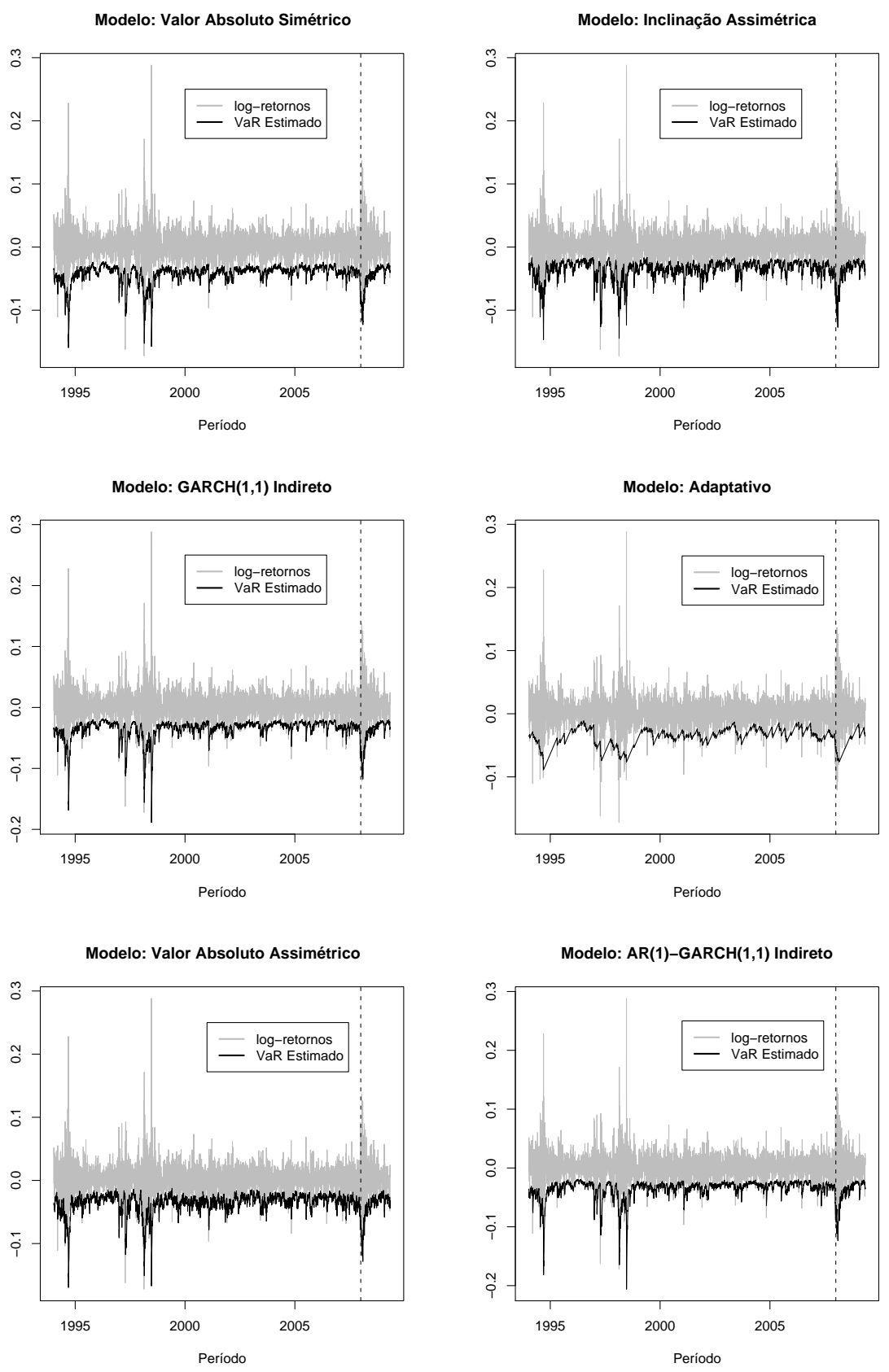

Figura 7.10: Gráficos de VaR estimados através dos modelos CAViaR. 


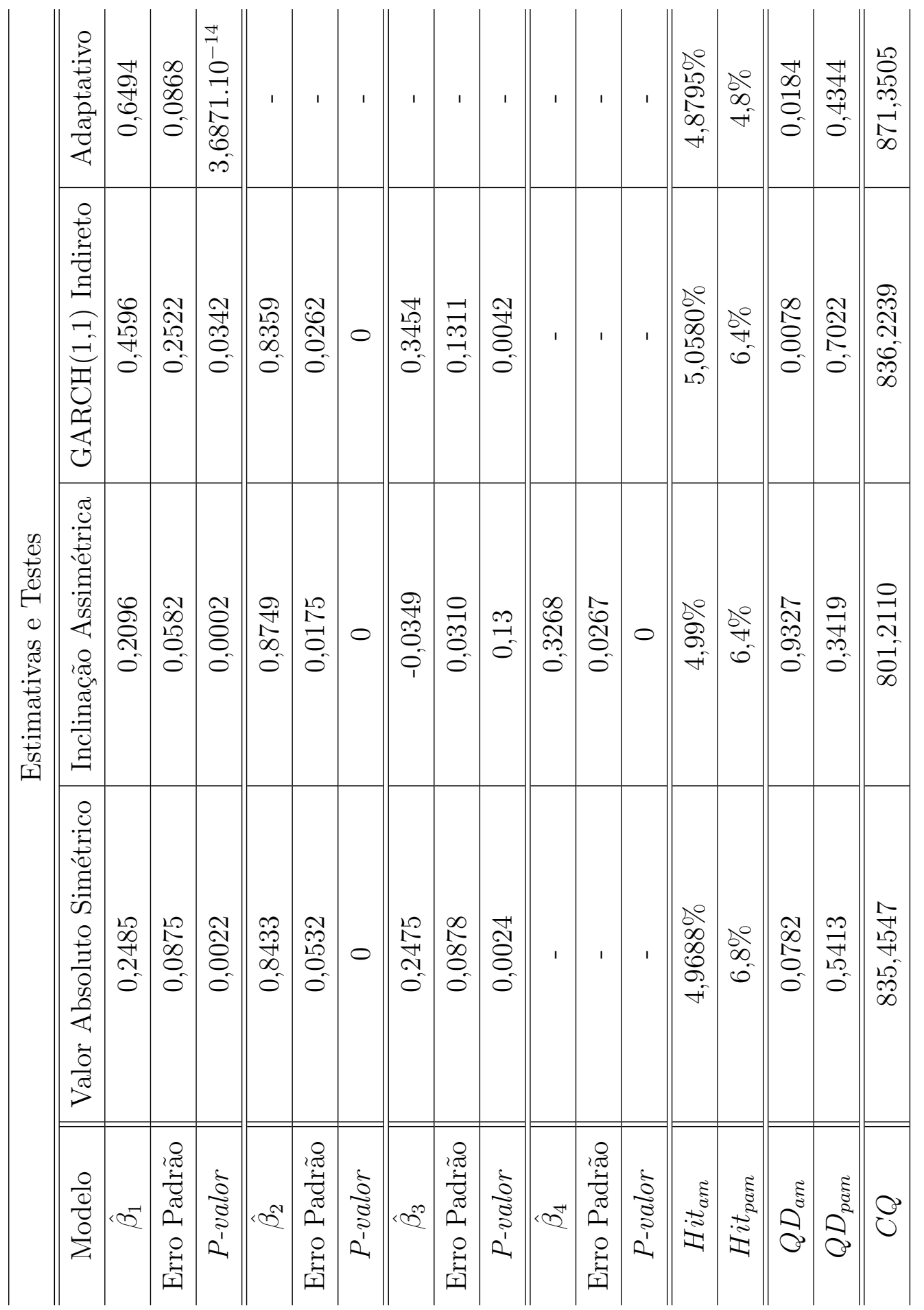

Tabela 7.5: Estimativas e Estatísticas dos Parâmetros Estimados Utilizando as Especificações do Modelo CAViaR. 


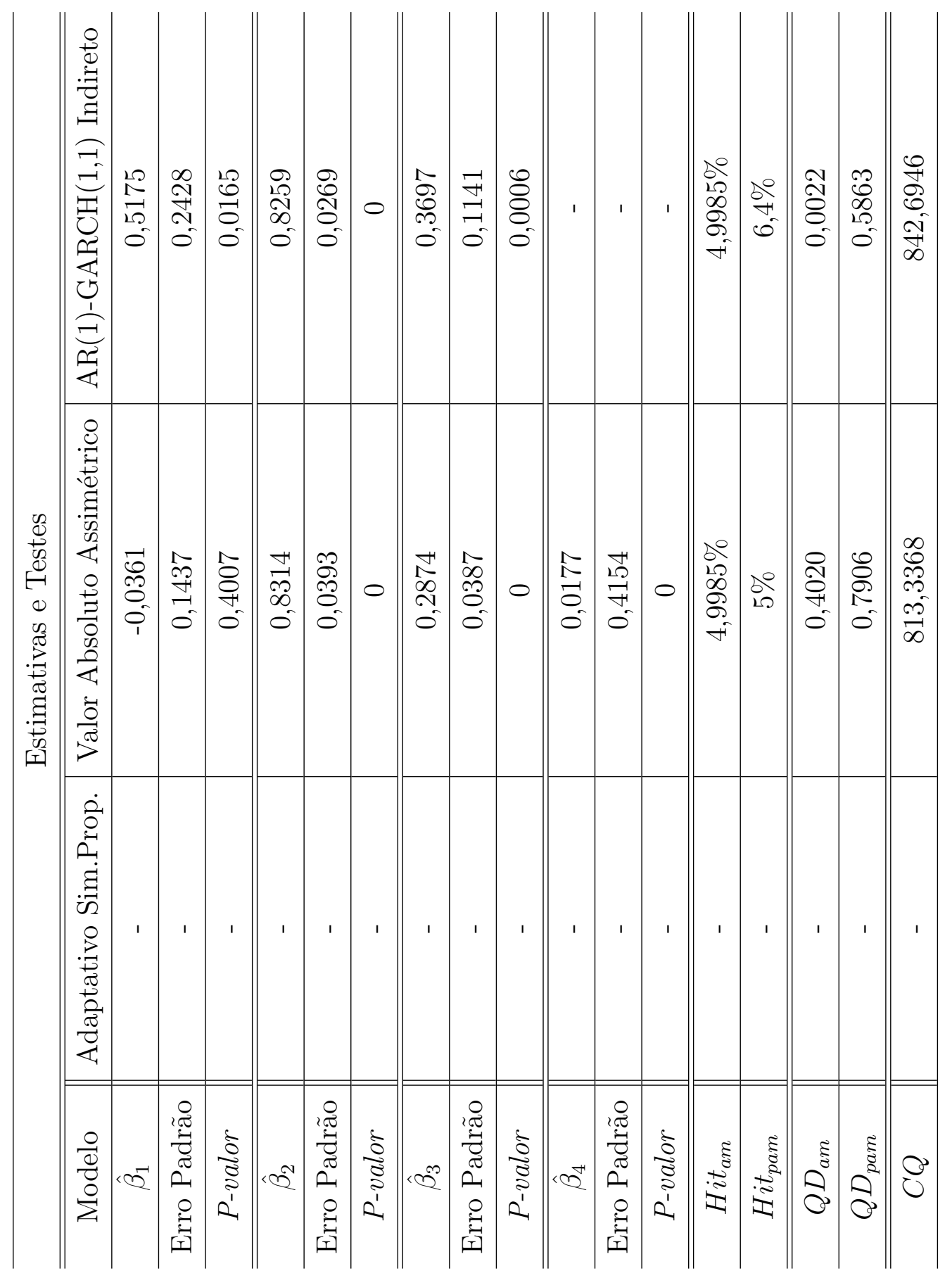

Tabela 7.6: Estimativas e Estatísticas dos Parâmetros Estimados Utilizando as Especificações do Modelo CAViaR. 
Dentre todos, este é o que apresenta menor Critério Quantílico, dando indícios de que seria o melhor modelo ajustado pra esta série. O parâmetro $\hat{\beta}_{3}$ com um $P$-valor $=0,13$ parece ser não significativo, indicando que as informações positivas não causam tanto impacto como as informações negativas. A proporção de Hits amostrais e pós-amostrais indicam que o modelo é adequado. Os testes de $Q D_{a m}$ e $Q D_{\text {pam }}$ também indicam que o modelo está ajustado corretamente à série.

A especificação $\mathbf{G A R C H}(\mathbf{1 , 1})$ Indireto resulta no seguinte modelo estimado:

$$
\widehat{\operatorname{VaR}}_{t}(0,05)=\left(0,4596+0,8359 \widehat{\operatorname{VaR}}_{t-1}^{2}(0,05)+0,3454 r_{t-1}^{2}\right)^{\frac{1}{2}}
$$

Este modelo possue boas proporções de $H i t_{a m}$ e $H i t_{\text {pam }}$, e todos os parâmetros significativos. Porém o valor do teste de $Q D_{a m}$ indica que o modelo não apresenta boa qualidade.

Para o Adaptativo foi adotado $k=10$ para fim de controle do valor do VaR no ajuste. Obtém-se então:

$$
\begin{aligned}
\widehat{V a R}_{t}(0,05) & =\widehat{V a R}_{t-1}(0,05)+ \\
& +0,6494\left\{\left[1+\exp \left[10\left(r_{t-1}-\widehat{V a R}_{t-1}(0,05)\right)\right]\right]^{-1}-0,05\right\}
\end{aligned}
$$

Este modelo é o que apresenta maior $C Q$ e menor $P$ - valor para o teste de $Q D_{a m}$, indicando ser o modelo menos adequado a essa série.

A especificação Valor Absoluto Assimétrico apresenta o modelo estimado:

$$
\widehat{V a R}_{t}(0,05)=-0,036+0,8314 \widehat{V a R}_{t-1}(0,05)+0,2874\left|r_{t-1}-0,0177\right| \text {. }
$$

Este é o que apresenta o segundo menor valor para o $C Q, \operatorname{com} \hat{\beta}_{1}$ não significativo. Já o $\hat{\beta}_{4}$, apesar de apresentar o maior erro padrão, é significativo. Na verdade, a inclusão deste parâmetro seria uma tentativa de tirar a influência que a magnitude de dados com valores atípicos poderia causar. É importante destacar que este modelo só se diferencia do modelo Valor Absoluto Simétrico por causa deste parâmetro $\beta_{4}$, e ao comparar os dois, é possível notar que a inclusão deste parâmetro apresenta melhora no valor do $C Q$ e dos testes de $Q D_{a m}$ e $Q D_{p a m}$. 
Ao modelo AR(1)-GARCH(1,1) Indireto, inicialmente foi necessário ajustar um modelo AR(1). Como o objetivo dos modelos CAViaR é não fazer suposições sobre a distribuição condicional dos retornos, para estimar o valor do $\phi_{1}$ utilizou-se, como estimador, o valor das autocovariâncias amostrais estimadas, através das equações de Yule-Walker, em que $\hat{\phi}_{1}=0,03506877$.

Logo,

$$
\begin{aligned}
\widehat{\operatorname{VaR}}_{t}(0,05)=0,035 r_{t-1}+[0,5175 & +0,8259\left(\widehat{\operatorname{VaR}}_{t-1}(0,05)-0,035 r_{t-2}\right)^{2}+ \\
& \left.+0,3697\left(r_{t-1}-0,035 r_{t-2}\right)^{2}\right]^{\frac{1}{2}}
\end{aligned}
$$

Este é o modelo que apresenta o segundo maior valor para o $C Q$ e parâmetros com o maior erro padrão, porém todos significativos ao modelo. Já os testes de $Q D_{a m}$ indica que o modelo não é adequado a série, o que já havia sido notado pelo valor do $C Q$.

O ajuste para o VaR utilizando modelo Adaptativo Simétrico Proporcional não foi possível para esta série. É importante destacar que modelo apresenta um comportamento diferente de todos os outros. De acordo com o visto em (4.5), apresenta a estrutura:

$$
\begin{aligned}
\operatorname{VaR}_{t}(\theta)=\operatorname{VaR} R_{t-1}(\theta)+ & \beta_{1} \max \left\{0,\left|r_{t-1}\right|-V_{a} R_{t-1}(\theta)\right\}- \\
& -\beta_{2} \min \left\{0,\left|r_{t-1}\right|-V_{a} R_{t-1}(\theta)\right\} .
\end{aligned}
$$

Note que $\left|r_{t-1}\right|-\operatorname{VaR}_{t-1}(\theta)$ é um resíduo, e no caso em que há uma variação muito grande dos valores dos retornos, não há nenhuma função que controle este resíduo, fazendo com que o valor do VaR aumente muito. Como o processo de estimação do modelo depende de valores simulados para o vetor $\boldsymbol{\beta}$, e tomou-se valores entre $(0,1)$, não houve convegência na estimação do modelo devido o comportamento da série.

De modo geral, ao se falar dos modelos CAViaR, como comentado no Capítulo 3, definir qual é o melhor modelo depende de qual cenário está se referindo. 
No caso da série do IBOVESPA, de acordo com os modelos ajustados, o mais indicado para estimar o valor do VaR seria o modelo Inclinação Assimétrica dado em (7.3), pois apresenta o menor valor para o $C Q$ e o melhor valor para o teste de $Q D_{a m}$. As proporções de Hits amostrais e pós-amostrais também indicam que o modelo é adequado.

\subsubsection{Modelos CARE}

Considerando as especificações definidas por Taylor (2008), primeiramente é necessário que se estime o valor do $\theta-$ quantil pelo $\tau$ - expectil de acordo com as idéias de Efron (1991).

Este processo de estimação só é possível devido ao fato de que para cada $\tau-$ expectil existe um correspondente $\theta$-quantil na amostra, em que não necessariamente $\tau$ seja igual a $\theta$. Todo o suporte teórico é feito e demonstrado por Jones (1994).

A Figura 7.11 apresenta o gráfico do $\theta$-quantil e $\tau$-expectil de acordo com os valores de $\theta$ e $\tau$ para a série de log-retornos.



Figura 7.11: Gráfico do $\theta$-quantil e $\tau$-expectil obtidos para a série de log-retornos. 
Pelo gráfico, é possível notar que $\tau$ apresenta valores mais extremos que os correspondentes valores de $\theta$.

Considerando a série de retornos do IBOVESPA, assim como nos modelos CAViaR, foram utilizadas para a estimação dos modelos os primeiros 3361 log-retornos, e de acordo com o procedimento detalhado no Capítulo 5, por (5.8), para $\theta=0,05$,

$$
\hat{\tau}=0,02436630 \text {. }
$$

Logo, seguindo o mesmo procedimento para a estimação dos modelos CAViaR, estima-se os parâmetros dos modelos, utilizando Mínimos Quadrados Assimétricos (MQA), ao invés do Critério Quantílico.

A Figura 7.12 apresenta os gráficos dos valores estimados para o VaR de acordo com as especificações do modelo CARE.

Os gráficos indicam que não há muita diferença entre as estimativas utilizando modelos CARE e CAViaR, a não ser para o modelo Adaptativo.

As Tabelas 7.7 e 7.8 apresentam o valor estimado, Erro Padrão, P-valor para cada um dos parâmetros, além da proporação de $H i t_{a m}$ e $H i t_{p a m}, Q D_{a m}, Q D_{\text {pam }}$, e também o valor do $M Q A$ e $C Q$ para cada modelo.

Assim como nos modelos CAViaR, todas as análises foram feitas considerando um nível de significância de $5 \%$.

Após a estimação do modelo, obteve-se ao seguinte modelo estimado para o VaR de acordo com especificação Valor Absoluto Simétrico:

$$
\hat{\mu}_{t}(0,024)=0,3352+0,804 \hat{\mu}_{t-1}(0,024)+0,2441\left|r_{t-1}\right| .
$$

De acordo com os valores estimados, os parâmetros $\beta_{1}$ e $\beta_{3}$ possuem $P$-valor indicando que são não significativos. Já o valor dos $H i t_{a m}$ e $H i t_{p a m}$ indicam que o modelo pode não ser adequado, pois os valores não estão em torno do $5 \%$ esperado. Os testes de $Q D_{a m}$ e $Q D_{\text {pam }}$ possuem $P$-valor bem pequenos, rejeitando a hipótese de que o modelo seja adequado. 

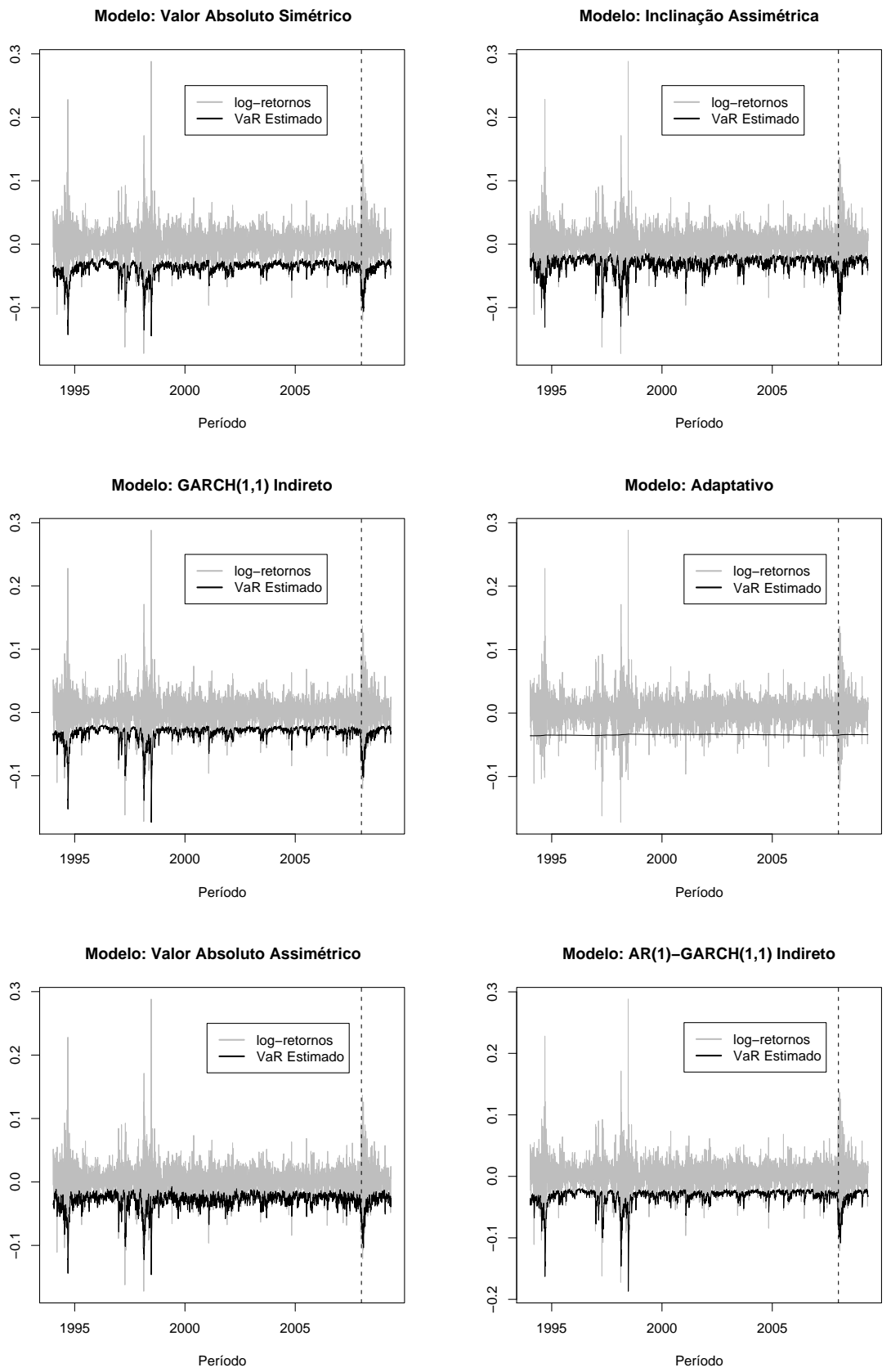

Figura 7.12: Gráficos de VaR estimados através dos modelos CARE. 


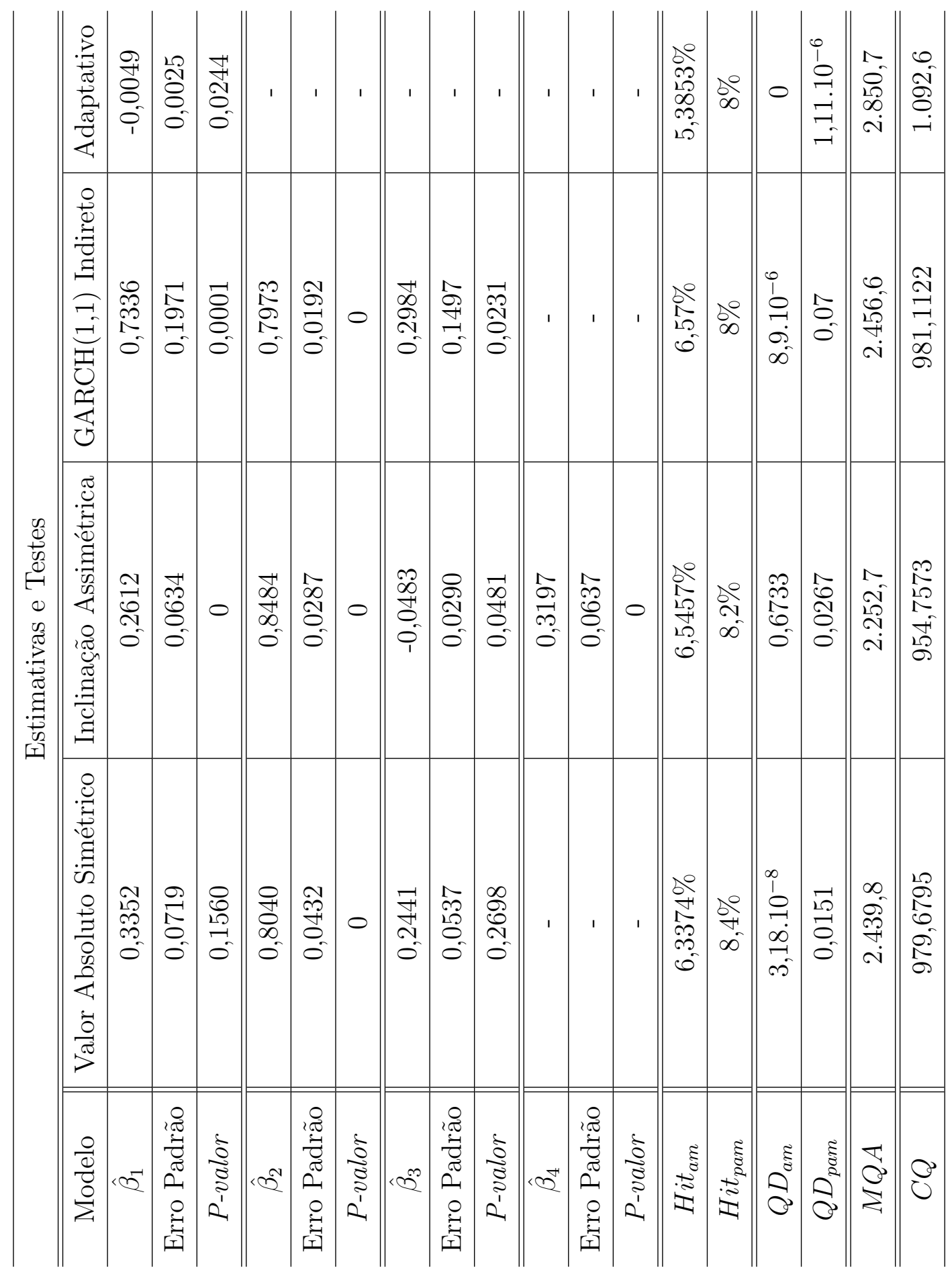

Tabela 7.7: Estimativas e Estatísticas dos Parâmetros Estimados Utilizando as Especificações do Modelo CARE. 


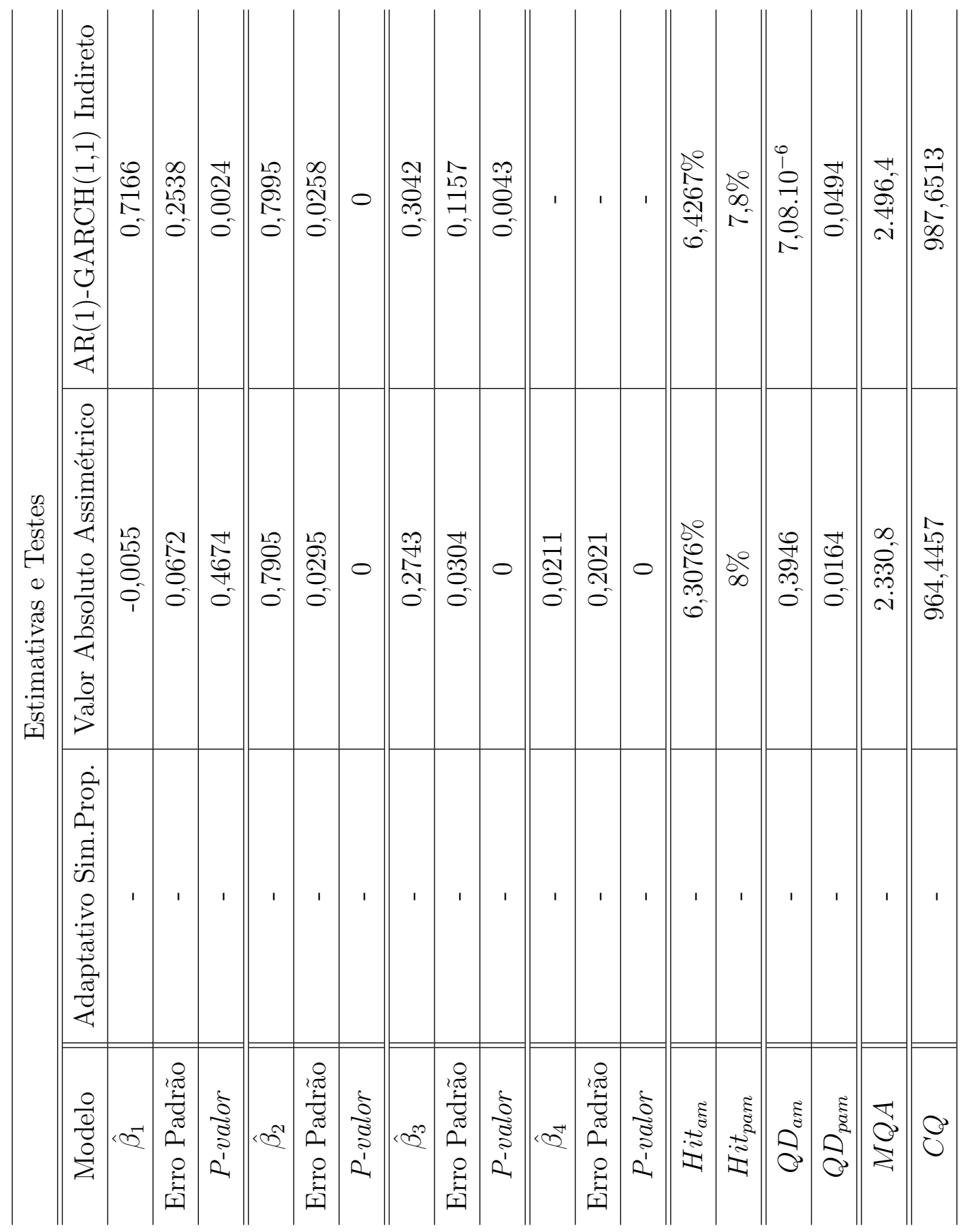

Tabela 7.8: Estimativas e Estatísticas dos Parâmetros Estimados Utilizando as Especificações do Modelo CARE. 
Através da especificação Inclinação Assimétrica, obtem-se o modelo estimado:

$$
\begin{aligned}
\hat{\mu}_{t}(0,024)=0,2612+0,8484 \hat{\mu}_{t-1}(0,024) & -0,0483 r t-1 I\left(r_{t-1}>0\right)+ \\
& +0,3197 r t-1 I\left(r_{t-1}<0\right) .
\end{aligned}
$$

Da mesma forma que os modelos CAViaR, esta é a especificação que apresenta o menor valor para o $M Q A$, além de o menor valor para o $C Q$. Todos os parâmetros estimados são significativos, além de apresentar o melhor valor para o teste de $Q D_{a m}$.

Já a especificação $\operatorname{GARCH}(\mathbf{1 , 1})$ Indireto possue o modelo estimado:

$$
\hat{\mu}_{t}(0,024)=\left(0,7336+0,7973 \hat{\mu}_{t-1}^{2}(0,024)+0,2984 r_{t-1}^{2}\right)^{\frac{1}{2}} .
$$

Apesar de apresentar $P$-valor indicando que todos os parâmetros são significativos, a proporção de $H i t_{a m}$ e $H i t_{p a m}$, os valores dos testes de $Q D_{a m}$ e $Q D_{p a m}$, e do $C Q$ e $M Q A$ indicam que o modelo não é adequado às observações.

O modelo Adaptativo, resulta no seguinte modelo estimado para o VaR:

$$
\begin{aligned}
\hat{\mu}_{t}(0,024) & =\hat{\mu}_{t-1}(0,024)- \\
& -0,0049\left\{\left[1+\exp \left[10\left(r_{t-1}-\hat{\mu}_{t-1}(0,024)\right)\right]\right]^{-1}-\theta\right\} .
\end{aligned}
$$

Este modelo é o que apresenta o maior valor para o $C Q$ e $M Q A$, além de possuir os piores valores para os testes de $Q D_{a m}$ e $Q D_{p a m}$, indicando que o modelo também não é adequado.

Este modelo, comparado aos modelos CAViaR, é o que mais difere. É possível notar que as estimativas são bem suaves, não acompanhando o comportamento dos log-retornos em períodos de instabilidade.

O modelo, utilizando a especificação Valor Absoluto Assimétrico é dado por:

$$
\hat{\mu}_{t}(0,024)=-0,0055+0,7905 \hat{\mu}_{t-1}(0,024)+0,2743\left|r_{t-1}-0,0211\right| .
$$

Este modelo é o que apresenta, dentre os estimados o segundo menor valor para o $C Q$ e $M Q A$, além de ser o segundo que apresenta o melhor valor para o teste $Q D_{a m}$, 
porém o teste de $Q D_{\text {pam }}$ possue valor indicando ser um modelo com qualidade não muito boa.

A especificação AR(1)-GARCH(1,1) Indireto apresenta o seguinte modelo estimado para o VaR:

$$
\begin{aligned}
\hat{\mu}_{t}(0,024)=0,035 r_{t-1}+[0,7166 & +0,7995\left(\hat{\mu}_{t-1}(0,024)-0,035 r_{t-2}\right)^{2}+ \\
& \left.+0,3042\left(r_{t-1}-0,035 r_{t-2}\right)^{2}\right]^{\frac{1}{2}} \cdot
\end{aligned}
$$

Este modelo também possue as proporções de $H i t_{a m}, H i t_{\text {pam }}$, além dos valores dos testes de $Q D_{a m}$ e $Q D_{\text {pam }}$ indicando que o modelo não é adequado.

Da mesma forma que no modelo CAViaR, não foi possíivel a estimação para o VaR através do modelo Adaptativo Simétrico Proporcional.

É importante destacar que o $\tau$-expectil, chamado de $\mu(\tau)$ é um estimador para o $\theta$ - quantil, que foi denominado ao longo do trabalho de $\operatorname{VaR}(\theta)$, sendo assim, ao fixar o valor $\theta=0,05$, para todas as especificações, as proporções de $H_{i t} t_{a m}, H_{\text {pam }}$ além dos testes de $Q D_{a m}$ e $Q D_{\text {pam }}$, e do $C Q$ foram calculados substituindo $\mu(\tau)$ por $\operatorname{VaR}(\theta)$.

Por exemplo, considere a especificação Valor Absoluto Simétrico dada em (7.9) utilizando modelos CARE, logo,

$$
\hat{\mu}_{t}(0,024)=0,3352+0,804 \hat{\mu}_{t-1}(0,024)+0,2441\left|r_{t-1}\right|
$$

pode ser escrito como

$$
\widehat{V a R}_{t}(0,05)=0,3352+0,804 \widehat{V a R}_{t-1}(0,05)+0,2441\left|r_{t-1}\right|
$$

Da mesma forma que os modelos CAViaR, o modelo que apresenta indícios de ser o mais adequado a série, apresentando melhores resultados foi o modelo Inclinação Assimétrica, dado em (7.10). 
Todas as especificações do modelo CARE apresentaram porcentagem de Hits pósamostrais com valores maiores que os valores apresentados para os modelos CAViaR. De fato, essas observações não fazem parte do processo de estimação, além pertencerem a um período de crise. Pelo fato da magnitude dos dados influenciarem mais nos modelos CARE, há um aumento da porcentagem dos Hit pam. O valor dos testes de Quantis Dinâmicos pós-amostrais também indicam que, para períodos de instabilidade, como os identificados na série do IBOVESPA, os modelos CARE, aparentemente são menos indicados que os modelos CAViaR, para o cálculo do VaR.

Considerando o fato de que as propriedades assintóticas dos estimadores de Mínimos Quadrados Assimétricos não foram discutidas e nem demostradas por Taylor (2008), em que ele usa as mesmas estruturas dos modelos CAViaR, além dos mesmos testes para seleção e adequação dos modelos estimados, surgem os modelos definidos e apresentados por Kuan, Yeh e Hsu (2009).

Para estas especificações, de forma mais prática, considerou-se a Especificação 3, $\operatorname{com} p=5$ e $q=5$, e a Especificação 4, $\operatorname{com} p=5$, em que $\theta=0,05$. Da mesma forma que os modelos CARE propostos por Taylor (2008), tem-se $\tau=0,024$.

O fato de considerar somente essas observações defasadas no tempo no processo de ajuste seria uma proposta de analisar a influência que as observações semanais podem causar ao modelo.

Sendo assim, após o ajuste do modelo, pelo $P$-valor que cada parâmetro estimado apresenta e pelo Erro Padrão, é possível analisar se são ou não significativos. Caso não sejam, é feito um novo ajuste em que os lags não significativos não são considerados ao modelo.

A Figura 7.13 apresenta as estimativas para o VaR, de acordo com a Especificação 3 e a Especificação 4, ambas utilizando os lags de ordem 5 para os logretornos defasados no tempo.

Os gráficos mostram que os valores estimados por essa nova proposta, aparentemente não diferem muito dos valores estimados segundo as idéias de Taylor (2008). 

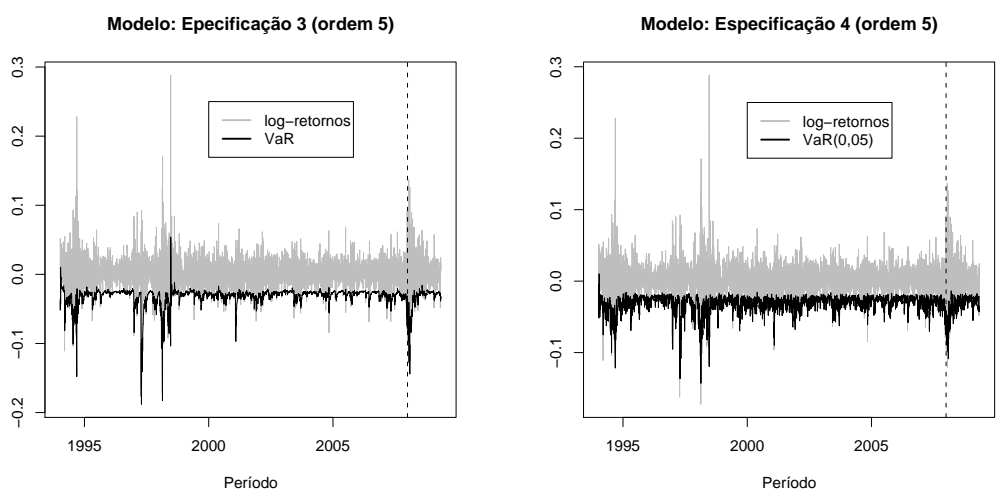

Figura 7.13: Estimativas para o VaR utilizando modelos CARE

Porém, para obter uma análise mais detalhada, são apresentados os parâmetros estimados nas Tabelas 7.9 e 7.10, além do Erro Padrão e do P-valor de cada um dos parâmetros.

Pelos valores observados nas tabelas, é possível notar que todos os parâmetros, em ambos os modelos são significativos, não havendo necessidade de reestimar o modelo.

A Tabela 7.11 apresenta os valores dos $H_{i t} t_{a m}, H_{\text {pam }}$ e $M Q A$ obtidos a partir da estimação dos modelos CARE. Os valores da tabela indicam que a proporção de $H i t_{a m}$ e $H_{i t_{\text {pam }}}$ estão bem próximos dos valores obtidos através dos modelos propostos por Taylor (2008), porém apresentam valores maiores para o $M Q A$.

Para fins de seleção e adequação do modelo, de acordo com o teste detalhado no Capítulo 5, testa-se Especificação 3 como o modelo sob $H_{0}$, contra Especificação 4 sob $H_{1}$.

A um nível de significância de 5\%, o teste apresentou a estatística

$$
Q=0,6783 \sim \chi_{(11)}^{2},
$$

com $P-$ valor $\approx 1$.

Já testando a Especificação 4 como o modelo sob $H_{0}$ contra a Especificação 


\begin{tabular}{c|c||c|c}
\hline \multicolumn{4}{c}{ Especificação 3 } \\
\hline \hline$\hat{a}_{0}(\tau)$ & 0,2344 & - & - \\
\hline Erro Padrão & 0,0081 & - & - \\
\hline$P-$ valor & 0 & - & - \\
\hline \hline$\hat{a}_{1}(\tau)$ & 0,9050 & - & - \\
\hline Erro Padrão & 0,0029 & - & - \\
\hline$P-$ valor & 0 & - & - \\
\hline \hline$\hat{b}_{1}(\tau)$ & $-0,0225$ & $\hat{\gamma}_{1}(\tau)$ & 0,0198 \\
\hline Erro Padrão & 0,0001 & Erro Padrão & 0,0002 \\
\hline$P-$ valor & 0 & $P-$ valor & 0 \\
\hline \hline$\hat{b}_{2}(\tau)$ & 0,0055 & $\hat{\gamma}_{2}(\tau)$ & 0,0305 \\
\hline Erro Padrão & 0,0001 & Erro Padrão & 0,0002 \\
\hline$P-$ valor & 0 & $P-$ valor & 0 \\
\hline \hline$\hat{b}_{3}(\tau)$ & $-0,0014$ & $\hat{\gamma}_{3}(\tau)$ & $-0,0240$ \\
\hline Erro Padrão & 0,0001 & Erro Padrão & 0,0002 \\
\hline$P-$ valor & 0 & $P-$ valor & 0 \\
\hline \hline$\hat{b}_{4}(\tau)$ & 0,0099 & $\hat{\gamma}_{4}(\tau)$ & 0,0244 \\
\hline Erro Padrão & 0,0001 & Erro Padrão & 0,0002 \\
\hline$P-$ valor & 0 & $P-$ valor & 0 \\
\hline \hline$\hat{b}_{5}(\tau)$ & $-0,0013$ & $\hat{\gamma}_{5}(\tau)$ & $-0,0165$ \\
\hline Erro Padrão & 0,0001 & Erro Padrão & 0,0002 \\
\hline$P-$ valor & 0 & $P-$ valor & 0 \\
\hline \hline
\end{tabular}

Tabela 7.9: Estimativas e Estatísticas dos Parâmetros Estimados Utilizando a Especificação 3 do Modelo CARE.

3, obteve-se a estatística:

$$
Q_{2}=1,1843 \sim \chi_{(12)}^{2},
$$




\begin{tabular}{c|c||c|c}
\hline \multicolumn{4}{c}{ Especificação 4} \\
\hline \hline$\hat{a}_{0}(\tau)$ & $-1,8544$ & - & - \\
\hline Erro Padrão & 0,0032 & - & - \\
\hline$P-$ valor & 0 & - & - \\
\hline \hline$\hat{b}_{1}(\tau)$ & $-0,0984$ & $\hat{\gamma}_{1}(\tau)$ & 0,4421 \\
\hline Erro Padrão & 0,0014 & Erro Padrão & 0,0014 \\
\hline$P-$ valor & 0 & $P-$ valor & 0 \\
\hline \hline$\hat{b}_{2}(\tau)$ & 0,0133 & $\hat{\gamma}_{2}(\tau)$ & 0,2443 \\
\hline Erro Padrão & 0,0014 & Erro Padrão & 0,0014 \\
\hline$P-$ valor & 0 & $P-$ valor & 0 \\
\hline \hline$\hat{b}_{3}(\tau)$ & 0,0291 & $\hat{\gamma}_{3}(\tau)$ & 0,2369 \\
\hline Erro Padrão & 0,0013 & Erro Padrão & 0,0014 \\
\hline$P-$ valor & 0 & $P-$ valor & 0 \\
\hline \hline$\hat{b}_{4}(\tau)$ & 0,1100 & $\hat{\gamma}_{4}(\tau)$ & 0,2966 \\
\hline Erro Padrão & 0,0013 & Erro Padrão & 0,0014 \\
\hline$P-$ valor & 0 & $P-$ valor & 0 \\
\hline \hline$\hat{b}_{5}(\tau)$ & 0,1097 & $\hat{\gamma}_{5}(\tau)$ & 0,2418 \\
\hline Erro Padrão & 0,0013 & Erro Padrão & 0,0014 \\
\hline$P-$ valor & 0 & $P-$ valor & 0 \\
\hline \hline
\end{tabular}

Tabela 7.10: Estimativas e Estatísticas dos Parâmetros Estimados Utilizando a Especificação 4 do Modelo CARE.

$\operatorname{com} P-$ valor $\approx 1$.

Os valores dos testes indicam que os dois modelos podem ser considerados como adequados neste cenário. Porém, visando um modelo que possua menos parâmetros e uma melhor proporção de $H_{i} t_{\text {pam }}$, o modelo mais indicado neste cenário, utilizando modelos CARE de acordo com a proposta de Kuan, Yeh e Hsu (2009), seria o modelo 


\begin{tabular}{c||c|c}
\hline Modelo & Especificação 3 & Especificação 4 \\
\hline \hline Hit $_{a m}$ & $6,5754 \%$ & $6,5457 \%$ \\
\hline Hit $_{\text {pam }}$ & $9 \%$ & $7,8 \%$ \\
\hline$M Q A$ & $2.730,6$ & $2.757,2$ \\
\hline \hline
\end{tabular}

Tabela 7.11: Hits Amostrais e Pós-amostrais e Valor do MQA.

descrito como Especificação 4, isto é:

$$
\begin{aligned}
\mu_{t}(0,024) & =-1,8544-0,0984 r_{t-1}^{+}+0,0133 r_{t-2}^{+}+0,0291 r_{t-3}^{+}+ \\
& +0,11 r_{t-4}^{+}+0,1097 r_{t-5}^{+}+0,4221 r_{t-1}^{-}+0,2443 r_{t-2}^{-}+ \\
& +0,2369 r_{t-3}^{-}+0,2966 r_{t-4}^{-}+0,2418 r_{t-5}^{-} .
\end{aligned}
$$

\subsection{Calculo do ES}

A seguir, são feitas estimações para o ES de acordo com as abordagens descritas e detalhadas no Capítulo 6.

\subsubsection{Abordagem Econométrica}

Utilizando a abordagem econométrica, assim como foi feito para os modelos de VaR, o modelo mais indicado foi o modelo (7.1) com $\epsilon_{t} \sim t-$ Student. Sendo assim, para o ajuste do ES com essa abordagem, faz-se a estimação do ES utilizando a distribuição t-Student.

A Figura 7.14 apresenta os valores estimados para ES, com $\theta=0,05$ juntamente com os valores estimados para o VaR utilizando abordagem econométrica.

Os valores estimados para o ES pela abordagem econométrica, por ser influenciado pela volatilidade, são aparentemente mais indicados para períodos de instabilidade. 


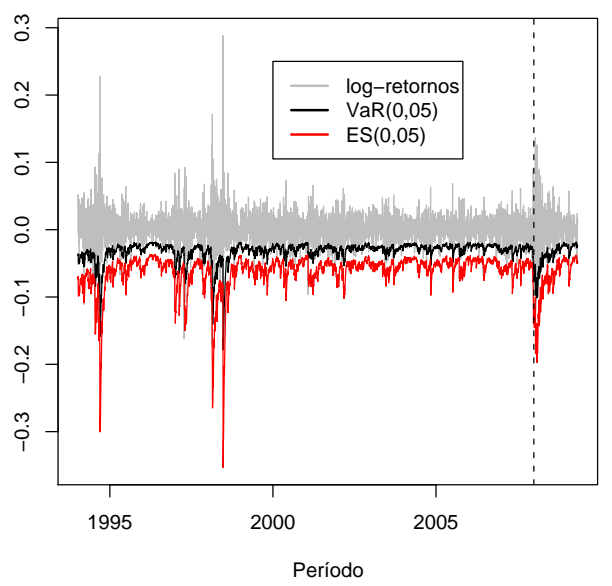

Figura 7.14: Estimativas para o VaR e ES, $\operatorname{com} \theta=0,05$.

\subsubsection{Teoria dos Valores Extremos}

A Figura 7.15 apresenta os valores estimados para VaR(TVE1) e ES(TVE1).

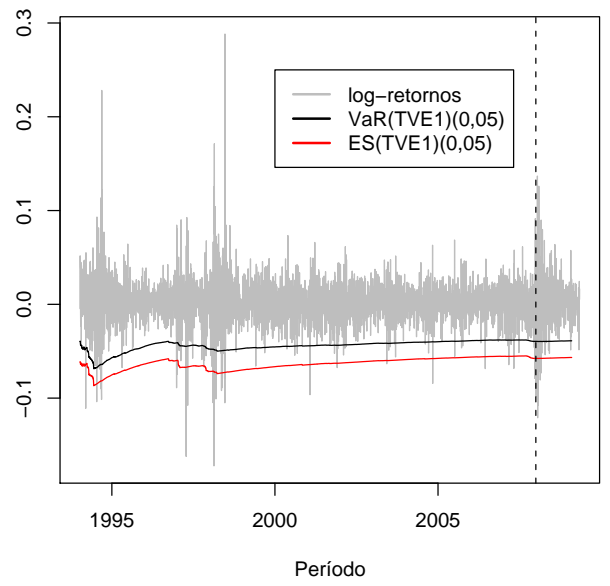

Figura 7.15: Estimativas para o VaR e ES, com $\theta=0,05$ utilizando TVE. 
Assim como os valores do VaR(TVE1), o ES(TVE1) também apresenta pouca variabilidade, podendo se tornar uma medida de risco não muito indicada em períodos de instabilidade, mas com poucas violações.

A Figura 7.16 apresenta os valores estimados para o ES(TVE2) e VaR(TVE2).

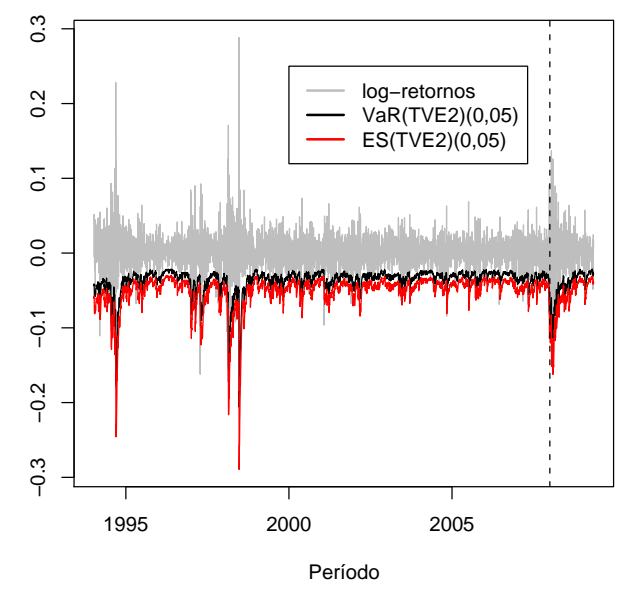

Figura 7.16: Estimativas para o VaR e ES, $\operatorname{com} \theta=0,05$ utilizando TVE.

Os valores estimados para ES(TVE2), assim como os valores estimados para ES, estão diretamente relacionados a volatilidade, diferentemente do método ES(TVE1), que funciona como uma janela móvel. Sendo assim, o método ES(TVE2) seria o mais indicado para períodos de crise.

Para fins de verificação de qual modelo é mais adequado neste cenário, faz-se o teste bootstrap descrito no Capítulo 6, com valores apresentados na Tabela 7.12.

\subsubsection{Modelos CARE}

De acordo com o demonstrado no Capítulo 6, a estimação do ES, utilizando modelos CARE é possível devido a ligação entre Mínimos Quadrados Assimétricos e a definição de ES. 
Considerando

$$
\widehat{E S}_{t}(\theta)=\left(1+\frac{\tau}{(1-2 \tau) \theta}\right) \hat{\mu}_{t}(\tau)-\frac{\tau}{(1-2 \tau) \theta} E\left(r_{t} \mid \mathcal{F}_{t-1}\right) .
$$

em que, de acordo com uma série estacionária,

$$
E\left(r_{t} \mid \mathcal{F}_{t-1}\right) \approx 0
$$

$\log \mathrm{O}$

$$
\widehat{E S}_{t}(\theta)=\left(1+\frac{\tau}{(1-2 \tau) \theta}\right) \hat{\mu}_{t}(\tau)
$$

Sendo assim, utilizando os modelos estimados para o $\tau$ - expectil de acordo com as especificações dos modelos CARE, conforme Taylor (2008), faz-se a estimação para o ES, cujas estimativas são apresentadas na Figura 7.17, em que se considerou $\theta=0,05$ e $\hat{\tau}=0,02436630$.

Para fins de verificação de qual modelo mais adequado neste cenário, os valores do teste bootstrap são apresentados, também, na Tabela 7.12. Observando os valores da Tabela 7.12, a um nível de 5\%, não há indícios de que algum dos modelos apresentados superestima sistematicamente o ES, porém é necessário que se apresente um modelo mais indicado neste cenário.

Já o método ES(TVE1), revelou-se de acordo com os gráficos vistos, o mais suave, não indicado para períodos de crise.

O método ES(TVE2) é o que apresenta maior P-valor, se comportando bem em períodos de crise.

É importante destacar que adotando a abordagem econométrica e TVE2 a modelagem da volatilidade é fundamental para a estimação do ES, o que não ocorre nos modelos CARE, nos quais ao invés de modelar a série de retornos, modela o $\tau-$ expectil e usa os resultados como estimativas para o $\theta$ - quantil. Logo, é possível notar que para esta série, todas as especificações são adequadas, pois todos os $P$ valores indicam a não rejeição de $H_{0}$, ou seja, que todos os excessos padronizados 

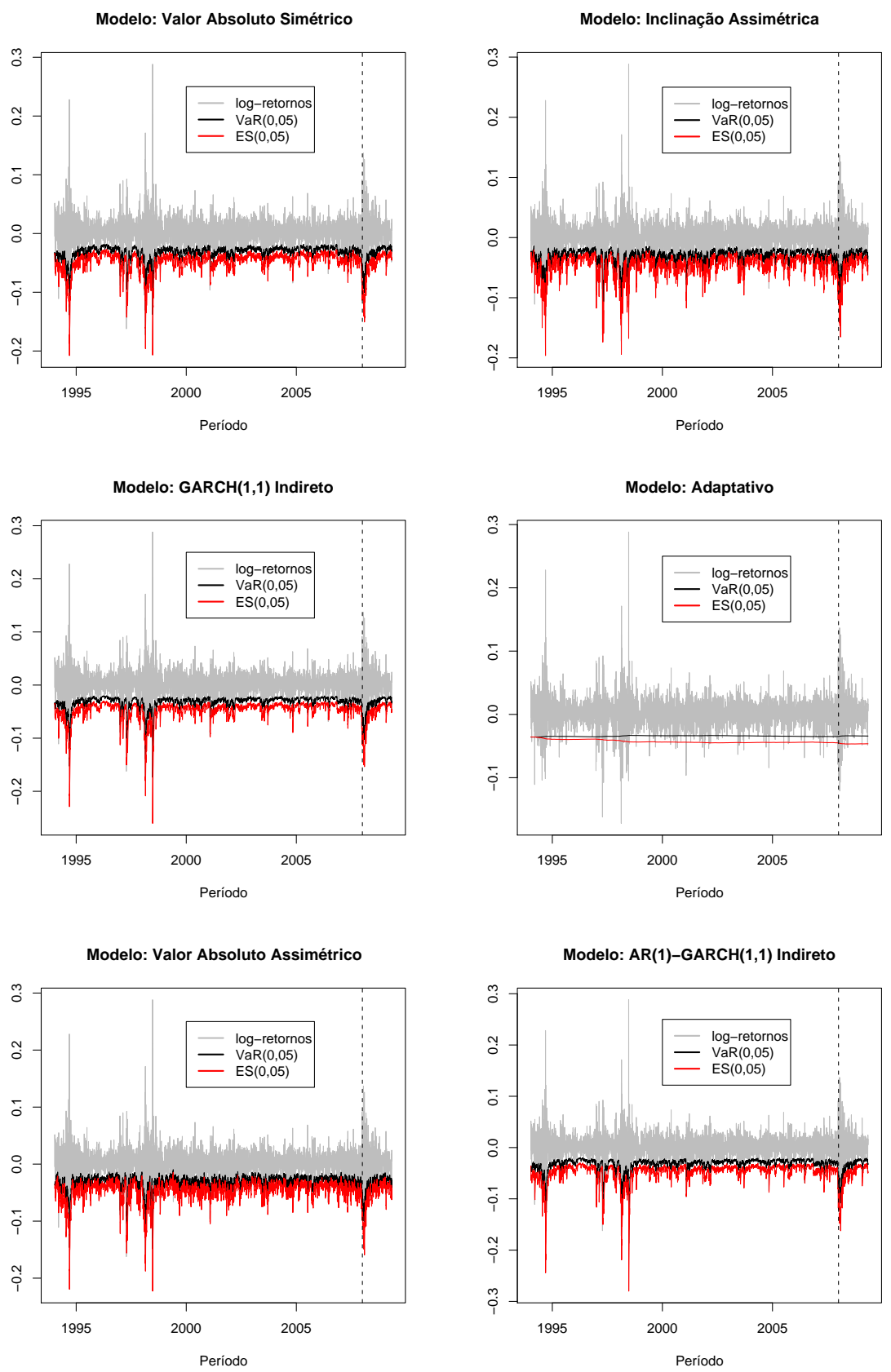

Figura 7.17: Gráficos de VaR e ES estimados através dos modelos CARE. 


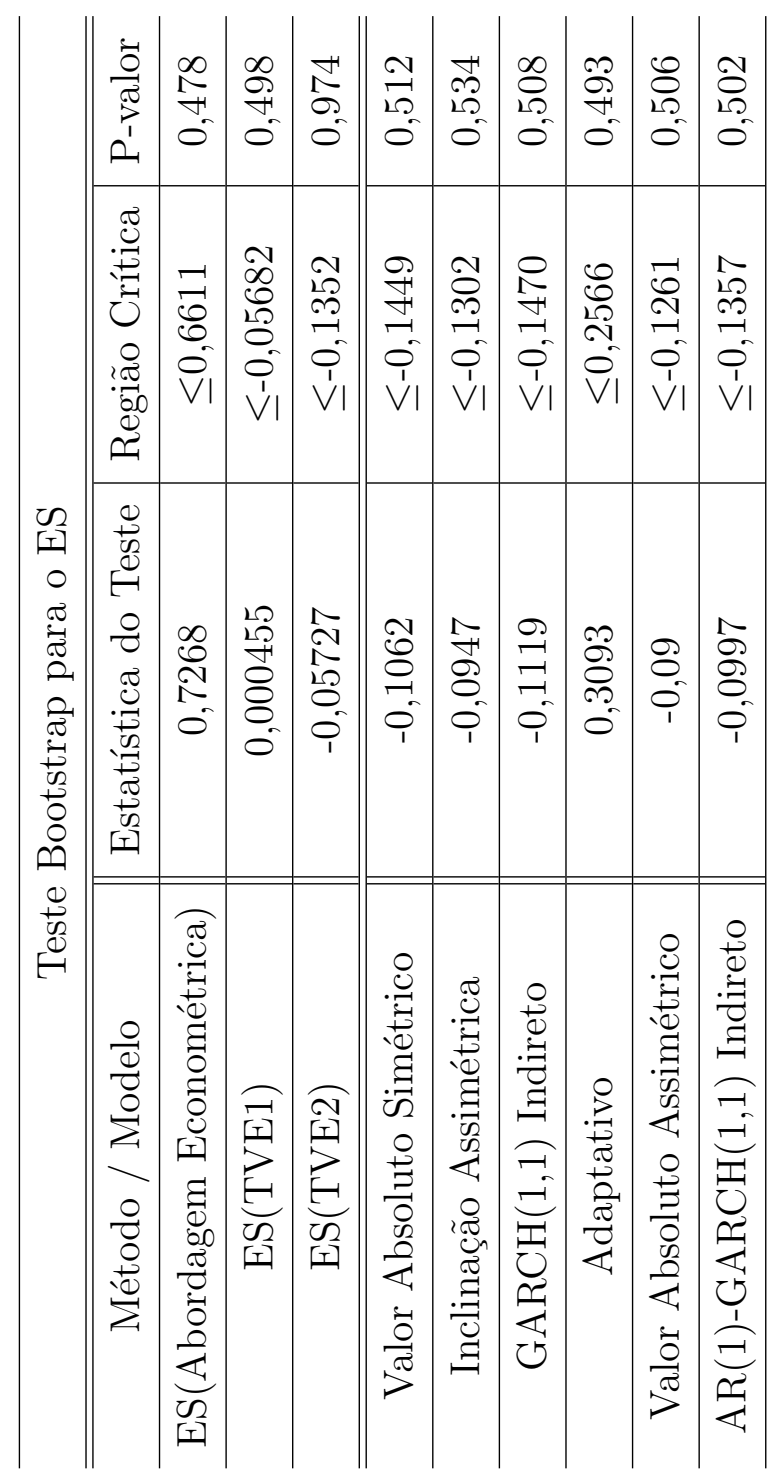

Tabela 7.12: Estatísticas do Teste Bootstrap para ES utilizando Abordagem Econométrica, TVE e modelos CARE.

tem média zero.

Muitos autores, visando estimar vários modelos e indicar o mais adequado, fazem 
vários ajustes para vários valores de $\theta$, para posições compradas e vendidas, em vários cenários, utilizando como critério de aceitação e adequação de modelo o número de séries que possuem P-valor indicando rejeição ou não de $H_{0}$, de acordo com a abordagem adotada.

No trabalho, utilizou-se somente a série do IBOVESPA, pois o objetivo principal foi apresentar modelos e abordagens visando dentro de cada cenário analisar qual o mais adequado. 


\section{Capítulo 8}

\section{Conclusões}

Devido a imposições do Comitê de Basiléia, os bancos e instituições financeiras tem aperfeiçoado e se preocupado mais em apresentar medidas de Risco cada vez mais precisas e adequadas em determinados cenários.

O trabalho apresentou e detalhou vários métodos e modelos para a mensuração dessas medidas visando comparar e obter as melhores estimativas para a série do IBOVESPA.

Para a estimação do VaR, utilizando a abordagem econométrica, notou-se que pelo fato da volatilidade estar incorporada ao cálculo do VaR, para períodos de instabilidade, o método mais adequado foi o que considera também a TVE, o qual denominou-se VaR(TVE2), pois foi o que apresentou a melhor proporção de valores em torno dos 5\%, apesar de apresentar um número de excessos acima do esperado, já que fixou-se um $\theta=0,05$ para posição comprada. A proposta da utilização da TVE nos modelos para mensuração do VaR, a partir da escolha de um limiar apropriado seria para obter medidas de risco que possam ser calculadas obtendo informação máxima das observações.

Já utilizando os modelos CAViaR, de acordo com os testes, proporção de $H i t_{a m}$ e $H i t_{\text {pam }}$, além do $C Q$ e $P$-valor dos testes de Quantis Dinâmicos, o melhor modelo neste cenário foi Inclinação Assimétrica, dado em (7.3). Estes modelos se 
tornaram tão competitivos para cálculo da medida de risco, quanto os mais simples. A diferença está no fato destes modelos CAViaR não fazerem suposição alguma sobre a distribuição dos retornos condicionais, o que pode evitar uma superestimação para o quantil se a suposição não for adequada, pois estes modelos modelam diretamente os quantis dessas observações.

De acordo com os modelos CARE, também utilizados para a estimação do VaR de acordo com Taylor (2008), o que apresentou melhores resultados foi também a Inclinação Assimétrica, dado em (7.10), porém para a proporção de Hit $t_{a m}$, Hit $_{\text {pam }}$ e valores dos testes de Quantis Dinâmicos para a série do IBOVESPA, não se tornou tão competitivo quanto os modelos CAViaR, pois a série apresenta período de instabilidade que influencia bem mais no processo de estimação destes modelos.

Seguindo a proposta de Kuan, Yeh e Hsu (2009), que discute e demonstra toda a teoria assintótica dos modelos CARE, a especificação que se apresenta como a mais adequada seria a Especificação 4 dada em (7.15), onde tomou-se $q=5$, com valores estimados dados pela Tabela 7.10. Estes modelos apresentaram proporção de $H i t_{a m} \mathrm{e}$ Hit $t_{\text {pam }}$ bem próximos dos obtidos para modelos CARE de acordo com Taylor (2008), o que em termos práticos, neste cenário, indica que toda a discussão é válida, porém são modelos bem mais complexos e que não apresentam uma melhora significativa na obtenção das medidas de risco.

Logo, para cálculo do VaR, neste cenário, de todos os modelos e métodos, o mais indicado seria o modelo CAViaR seguindo a estrutura Inclinação Assimétrica, dada em (7.3).

Após toda a discussão de medidas de risco coerente, surge o Expected Shortfall, que analisa o comportamento dos retornos que estão acima ou abaixo do valor do VaR, dependendo da posição.

O processo de estimação para o ES segue os mesmos moldes dos modelos definidos para VaR, porém não pode ser estimado por modelos CAViaR, pois estes não apresentam suporte teórico para esta mensuração.

Utilizando a abordagem econométrica, assim como no VaR, o método que apre- 
sentou melhores resultados foi o ES(TVE2). Dos modelos que utilizam expectil, definidos como CARE, todos eles apresentaram resultados bem semelhantes.

De forma geral, todos os métodos e modelos definidos, detalhados e discutidos são válidos para o cáculo do VaR e do ES, mas como comentado anteriormente, o objetivo principal do trabalho foi apresentar técnicas, não definidas como as melhores, mas as que apresentam resultados mais adequados dentro de determinados cenários, além de mostrar como modelos CAViaR e CARE podem se tornar tão precisos e adequados como os métodos mais simples e mais utilizados no mercado, porém sem fazer suposição alguma sobre a distribuição condicional das observações. 


\section{Apêndice A}

\section{Distribuição T-Student Generalizada Assimétrica.}

Para a distribuição t-Student com média zero, desvio padrão 1 e $v$ graus de liberdade, definida em (2.9), de acordo com Fernándes e Steel (1998), $f(t ; \nu, \psi)$ definida em (2.11) é dada por:

$$
\begin{aligned}
f(t ; v, \psi) & =\frac{2 \psi}{\psi^{2}+1}\left\{\frac{\Gamma\left(\frac{v+1}{2}\right)}{\Gamma\left(\frac{1}{2}\right)(v \pi)^{\frac{1}{2}}}+\left(1+\frac{\left(\frac{t}{\psi}\right)^{2}}{v}\right)^{-\left(\frac{v+1}{2}\right)} I_{[0,+\infty)}(t)+\right. \\
& \left.+\frac{\Gamma\left(\frac{v+1}{2}\right)}{\Gamma\left(\frac{1}{2}\right)(v \pi)^{\frac{1}{2}}}+\left(1+\frac{(t \psi)^{2}}{v}\right)^{-\left(\frac{v+1}{2}\right)} I_{(-\infty, 0)}(t)\right\}= \\
& =\left(\frac{2 \psi}{\psi^{2}+1} \frac{\Gamma\left(\frac{v+1}{2}\right)}{\Gamma\left(\frac{v}{2}\right)(v \pi)^{\frac{1}{2}}}\right)+\left\{\left(1+\frac{\left(\frac{t}{\psi}\right)^{2}}{v}\right)^{-\left(\frac{v+1}{2}\right)} I_{[0,+\infty)}(t)+\right. \\
& \left.+\left(1+\frac{(t \psi)^{2}}{v}\right)^{-\left(\frac{v+1}{2}\right)} I_{(-\infty, 0)}(t)\right\} .
\end{aligned}
$$


114APÊNDICE A. DISTRIBUIÇÃO T-STUDENT GENERALIZADA ASSIMÉTRICA.

Seja

$$
\begin{aligned}
C & =\left(\frac{2 \psi}{\psi^{2}+1} \frac{\Gamma\left(\frac{v+1}{2}\right)}{\Gamma\left(\frac{v}{2}\right)(v \pi)^{\frac{1}{2}}}\right)=\left(\frac{2}{\psi+\frac{1}{\psi}}\right) \frac{\Gamma\left(\frac{v+1}{2}\right)}{\Gamma\left(\frac{v}{2}\right)\left(\frac{v}{2}\right)^{\frac{1}{2}}(2 \pi)^{\frac{1}{2}}} \\
& =\left(\frac{2}{\psi+\frac{1}{\psi}}\right) \frac{1}{\left(\frac{v}{2}\right)^{\frac{1}{2}}} \frac{\Gamma\left(\frac{v+1}{2}\right)}{\Gamma\left(\frac{v}{2}\right)(2 \pi)^{\frac{1}{2}}}=\left[\left(\psi+\psi^{-1}\right) 2^{-1}\left(\frac{v}{2}\right)^{\frac{1}{2}} B\left(\frac{v}{2}, \frac{1}{2}\right)\right]^{-1} .
\end{aligned}
$$

Fazendo $\frac{v}{2}=\nu \Rightarrow v=2 \nu$,

$$
\begin{aligned}
C & =\left[\left(\psi+\psi^{-1}\right) 2^{-1}(\nu)^{\frac{1}{2}} B\left(\nu, \frac{1}{2}\right)\right]^{-1} \mathrm{e} \\
f(t ; \nu, \psi) & =C\left\{\left(1+\frac{\left(\frac{t}{\psi}\right)^{2}}{2 \nu}\right)^{-\left(\nu+\frac{1}{2}\right)} I_{[0,+\infty)}(t)+\left(1+\frac{(t \psi)^{2}}{2 \nu}\right)^{-\left(\nu+\frac{1}{2}\right)} I_{(-\infty, 0)}(t)\right\}= \\
& =C\left\{\left(1+\frac{\left(\frac{t}{\sqrt{2} \psi}\right)^{2}}{\nu}\right)^{-\left(\nu+\frac{1}{2}\right)} I_{[0,+\infty)}(t)+\left(1+\frac{\left(\frac{t \psi}{\sqrt{2}}\right)^{2}}{\nu}\right)^{-\left(\nu+\frac{1}{2}\right)} I_{(-\infty, 0)}(t)\right\} .
\end{aligned}
$$

Tomando $\frac{T}{\sqrt{2}}=Z$, em que $T \sim t(0,1, v)$, a densidade da v.a. $Z$ é definida como uma t-Student com parâmetros de posição $\mu$ e escala $\sigma^{2}$, pois $Z=\mu+\sigma T$, onde $\mu=0$ e $\sigma=\frac{1}{\sqrt{2}}$.

Assim, com o parâmetro de assimetria indexado, $Z \sim t-$ Student Generalizada Assimétrica, em que:

$$
f(z ; \nu, \psi)=C\left\{\left(1+\frac{\left(\frac{z}{\psi}\right)^{2}}{\nu}\right)^{-\left(\nu+\frac{1}{2}\right)} I_{[0,+\infty)}(z)+\left(1+\frac{(z \psi)^{2}}{\nu}\right)^{-\left(\nu+\frac{1}{2}\right)} I_{(-\infty, 0)}(z)\right\} .
$$




\section{Apêndice B}

\section{Medidas de Risco}

De acordo com McNeil, Frey e Embrechts (2005) e Artzner, Delbaen, Eber e Heath (1999), fixando um espaço de probabilidade $(\Omega, \mathcal{F}, \mathcal{P})$ e um horizonte de tempo $h$, seja $L^{0}(\Omega, \mathcal{F}, \mathcal{P})$ o conjunto de todas as variáveis aleatórias em $(\Omega, \mathcal{F})$ finitas.

Chama-se de Risco Financeiro o conjunto de todas as variáveis aleatórias $\mathcal{M} \subset$ $L^{0}(\Omega, \mathcal{F}, \mathcal{P})$ que são interpretadas como as perdas de um portfóleo em algum período $h$. Assume-se que $\mathcal{M}$ é uma cônica convexa, isto é:

- Seja $L_{1} \in \mathcal{M}$ e $L_{2} \in \mathcal{M} \Rightarrow\left(L_{2}+L_{2}\right) \in \mathcal{M}$;

- Para todo $\lambda>0, \lambda L_{1} \in \mathcal{M}$;

Medidas de Risco são funções de valores reais $\omega: \mathcal{M} \rightarrow \mathbb{R}$, definidas em cones convexos de v.a., satisfazendo certas propriedades. Assim, $\omega(L)$ é a quantia de capital que deverá ser adicionada a uma posição com perda dada por L, logo essa posição torna aceitável para um controlador de risco externo ou interno.

Associando uma medida de risco e aceitabilidade, tem-se a definição:

Definição [1]: O conjunto de aceitação associado a uma medida de risco $\omega$ é dada por um cojunto $\mathcal{A}_{\omega}$ definido por:

$$
\mathcal{A}_{\omega}=\{L \in \mathcal{M} \mid \omega(L) \leq 0\}
$$


As medidadas de Risco $\omega$ apresentam os seguintes axiomas:

Axioma[1] - Translação Invariante: Para todo $L \in \mathcal{M}$ e todo $r \in \mathbb{R}$,

$$
\omega(L+r)=\omega(L)+r .
$$

$\mathrm{O}$ axioma indica que ao adicionar ou subtratir uma determinada quantia $r \in \mathbb{R}$ a uma posição com perda $L$, a quantia de capital exigida é aumentada ou diminuída em exatamente a mesma quantia.

Considere uma posição com perda $L$, e $\omega(L)>0$, seja

$$
L^{\prime}=L-\omega(L),
$$

logo sabendo que $\omega(L) \in \mathbb{R}$, faz-se

$$
\omega\left(L^{\prime}\right)=\omega(L-\omega(L))=\omega(L)-\omega(L)=0 .
$$

Axioma[2] - Subaditividade: Para todo $L_{1}$ e $L_{2} \in \mathcal{M}$,

$$
\omega\left(L_{1}+L_{2}\right) \leq \omega\left(L_{1}\right)+\omega\left(L_{2}\right) .
$$

Este axioma indica que o risco pode ser reduzido por uma diversificação, ou seja, um investidor poderá reduzir o risco de investimento de um portfólio operando diferentes quantias para cada posição do portfólio, fazendo uma descentralização do sistema de gerenciamento de risco.

Considerando como exemplo, duas carteiras de negociação com perdas $L_{1}$ e $L_{2}$. Dado que o gerenciador de risco deseja medir $\omega(L)$, onde $L$ é o risco de perda geral, $L=L_{1}+L_{2}$ e que $M$ seja menor que $L$, ao usar uma medida de risco subaditiva $\omega$, ele pode escolher limites $M_{1}$ e $M_{2}$ tais que $M_{1}+M_{2} \leq M$, e impor à cada uma dessas carteiras que $\omega\left(L_{i}\right) \leq M_{i}$, logo pela subaditividade de $\omega, \omega(L) \leq M_{1}+M_{2} \leq M$.

Axioma[3] - Positividade Homogênea: Para todo $L \in \mathcal{M}$ e $\lambda \in \mathbb{R}$,

$$
\omega(\lambda L)=\lambda \omega(L) .
$$


O axioma é facilmente justificado pela subaditividade. Seja $n \in \mathbb{N}$ :

$$
\omega(n L)=\omega(L+\ldots+L) \leq n \cdot \omega(L)
$$

Considerando que não haja diversificação entre as perdas do portfólio, a igualdade $\omega(n L)=n \cdot \omega(L)$ é obtida naturalmente.

As medidas de risco que apresentam os axiomas [2] e [3] são ditas convexas em $\mathcal{M}$.

Axioma[4] - Monotonicidade: Para $L_{1}$ e $L_{2} \in \mathcal{M}$, tal que $L_{1} \leq L_{2}$ quase certamente,

$$
\omega\left(L_{1}\right) \leq \omega\left(L_{2}\right) .
$$

Para uma medida de Risco que satifaz os axiomas [2] e [3] e estabelecendo a condição

$$
\forall L \leq 0, \omega(L) \leq \omega(0)=0, \text { pois } \omega(0)=\omega(n .0)=n . \omega(0)=0 .
$$

Sendo $L_{1} \leq L_{2} \Rightarrow L_{1}-L_{2} \leq 0, \operatorname{logo} \omega\left(L_{1}-L_{2}\right) \leq 0$.

Então

$$
\begin{gathered}
\omega\left(L_{1}\right)=\omega\left(L_{1}-L_{2}+L_{2}\right) \leq \underbrace{\omega\left(L_{1}-L_{2}\right)}_{\leq 0}+\omega\left(L_{2}\right) \leq \omega\left(L_{2}\right) \\
\therefore \omega(L 1) \leq \omega\left(L_{2}\right) .
\end{gathered}
$$

Do ponto de vista econômico, o axioma traz um resultado óbvio, pois posições com maiores perdas, requerem maiores riscos de capital.

De acordo com diversos cenários, é possível calcular diversas medidas de Risco, e existem aquelas classificadas como coerentes.

\section{Definição [2]: Medida de Risco Coerente}

Uma medida de Risco $\omega$ definida em uma cônica convexa $\mathcal{M}$ é dita coerente em $\mathcal{M}$ se satisfizer os quatro axiomas acima descritos e especificados. 


\section{Apêndice $\mathrm{C}$}

\section{Método da Aceitação-Rejeição}

Em muitos casos, simular valores de uma certa distribuição $F($.$) se torna impossível$ por métodos usuais, como o da transformada inversa. Nestes casos, analisar e explorar as propriedades probabilísticas dessas disribuições é algo bem complicado.

O Método da Aceitação-Rejeição pertence a uma classe de métodos que só exige saber a forma funcional da densidade $f$ de iteresse, sem um profundo estudo analítico. A idéia do método é o uso de uma densidade $g$ auxiliar de fácil simulação.

Neste caso, considere uma densidade $f$ tal que

$$
f(x)=\int_{0}^{f(x)} 1 d u,
$$

e uma v.a. uniforme $U$. Logo, $f$ aparece como a densidade marginal em $X$ da distribuição conjunta

$$
(X, U) \sim U(x, u): 0<u<f(x),
$$

desde que $U$ não seja diretamente relacionada ao problema original, onde é chamada de variável auxiliar.

Utilizando a densidade conjunta dada em (C.1), é possível gerar valores dessa distribuição através de uma v.a. uniforme construída no conjunto

$$
\{(x, u): 0<u<f(x)\},
$$


com $f$ sendo a função distribuição marginal de interesse da v.a. $X$, ou seja, gerando os valores do conjunto dado em (C.2) gera-se valores de $X$ com fdp f. Este fato é enfatizado pelo seguinte teorema:

\section{Teorema Fundamental da Simulação - Simulando}

$$
X \sim f(x)
$$

é equivalente a simular

$$
(X, U) \sim U(x, u): 0<u<f(x)
$$

O teorema é considerado como fundamental no sentido de representação formal, mas em alguns casos a simulação do par uniforme $(X, U)$ não é algo simples e direto. Por exemplo, é possível simular $X \sim F(x)$ e $U \mid X=x \sim U(0, f(x))$, o que em alguns casos resulta na obtenção de valores ruins, devido ao fato da distribuição uniforme ser simétrica.

A solução é simular valores $(X, U)$ em um intevalo maior, no qual a simulação é mais fácil, e então obter os valores que satisfazem as condições desejadas.

Por exemplo, suponha que

$$
\int_{a}^{b} f(x) d x=1
$$

em que $f(x)<m, \forall x \in(a, b)$. Então pode-se simular $(Y, U) \sim U([a, b],[0, m])$ e obter os pares $(y, u)$ tais que $0<u<f(y)$. 


\section{Apêndice D}

\section{Teste Bootstrap}

A idéia do teste é verificar, sem usar cálculos analíticos qual o comportamento da estatística de interesse, sem fazer suposições sobre a distribuição dos dados.

No, trabalho, por exemplo, ao considerar os excessos, deseja-se saber se a média desses excessos é, ou não, igual a zero. Optamos por utilizar o procedimento Bootstrap, apresentado por Efron e Tibshirani (1993), que consiste nos seguintes passos:

- Considere $\underline{x}=x_{1}, x_{2}, \ldots, x_{n}$ observações independentes;

- Defina a estatística $t($.$) de interesse, no caso, a média;$

- Gere um número B de amostras bootstrap, independentes, obtendo $\underline{x}^{* 1}, \underline{x}^{* 2}, \ldots, \underline{x}^{* B}$;

- Calcule as réplicas $t\left(\underline{x}^{* 1}\right), t\left(\underline{x}^{* 2}\right), \ldots, t\left(\underline{x}^{* B}\right)$ de cada amostra;

- Faça a distribuição empírica das réplicas com a finalidade de saber o comportamento das mesmas;

- A um nível de confiança $\alpha$, obtém-se a região crítica através do histograma da estatística calculada para as réplicas, indicando a região de rejeição para $H_{0}$.

- Calcula-se, então, $t(\underline{x})$ e observa-se se a estatística pertence ou não a região crítica. 


\section{Apêndice E}

\section{Ajuste do Modelo ARMA-TARCH}

Os modelos ARCH e GARCH tratam simetricamente os retornos, pois a volatilidade é uma função quadrática dos mesmos, sendo assim, visando incorporar o efeito assimétrico que o sinal dos retornos causam na modelagem da volatilidade, utiliza-se também os modelos TARCH("threshold ARCH"), que é um caso particular do modelo ARCH não-linear.

Sendo assim, a média e a volatilidade agora, dado que $r_{t}=\mu_{t}+\sigma_{t} \epsilon_{t}$, possuem as seguintes estruturas:

$$
\begin{aligned}
r_{t} & =\phi_{0}+\sum_{i=1}^{p} \phi_{i} r_{t-i}-\sum_{j=1}^{q} \theta_{j} a_{t-j}+a_{t} \\
a_{t} & =\sigma_{t} \epsilon_{t} \\
\sigma_{t}^{2} & =\omega+\alpha_{1} a_{t-1}^{2}+\gamma S_{t-1} a_{t-1}^{2}+\beta_{1} \sigma_{t-1}^{2}
\end{aligned}
$$

em que

$$
S_{t}=\left\{\begin{array}{l}
1, \text { se } a_{t}<0 \text { ("bad news") } \\
0, \text { se } a_{t} \geq 0 \text { ("good news") }
\end{array}\right.
$$

Se $\gamma \neq 0$, há um impacto de informação assimétrica. Espera-se que $\gamma>0$ para que "bad news"tenha um impacto maior. 
Sendo assim, o melhor modelo ajustado para a série do IBOVESPA, segundo os critérios AIC e BIC é o modelo $\mathrm{AR}(10)-\mathrm{TARCH}(1,1)$, cujo os valores ajustados, estatísticas e P-valores são mostrados na Figura E.1.

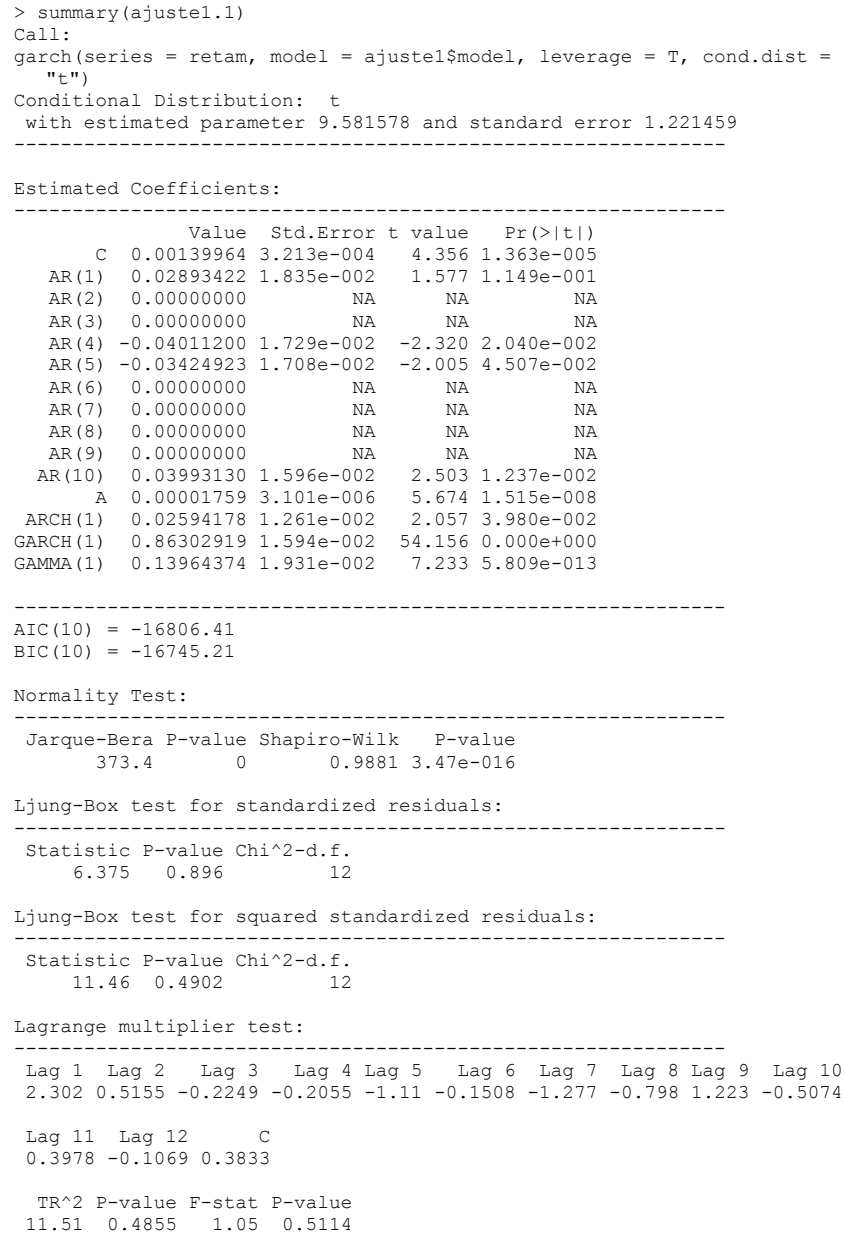

Figura E.1: Ajuste do modelo AR(10)-TARCH(1,1)

Os valores dos testes Ljung-Box para os resíduos padronizados e quadrado dos resíduos padronizados indicam que o modelo está bem ajustado à série. 


\section{Referências Bibliográficas}

Acerbi, C. and Tasche, D. (2002), On the Coherence of Expected Shortfall, Journal of Banking and Finance, 26, 1487-1503. 15, 62

Artzner, P., Delbaen, F., Eber, J. M. and Heath, D. (1999), Coherent Measures of Risk, Mathematical Finance, 9, 203-228. 61, 115

Coles, S. (2001), An Introduction to Statistical Modeling of Extreme Value, Springer, London. 22, 26

Efron, B. (1991), Regression Percentiles Using Asymmetric Squared Error Loss, Estatistica Sinica, 1, 93-125. 90

Efron, B. and Tibshirani, R. (1993), An Introduction to the Bootstrap, Chapman and Hall, New York . 121

Engle, R. F. and Manganelli, S. (2004), CAViaR: Conditional Autoregressive Value at Risk by Regression Quantiles, Journal of Business and Economic Statistics, 22, 367-381. 2, 31, 33, 39, 41, 42, 43, 83

Fernándes, C. and Steel, M.,F.,J. (1998), On Bayesian Modeling of Fat Tails and Skewness, Journal of the American Statistical Association, 93, 359-371. 1, 12, 113

Jones, M. C. (1994), Expectiles and M-quantiles are Quantiles, Statistics and Probability Letters, 20, 149-153. 90 
Koenker, R. and Basset, G. (1978), Regression Quantiles, Econometrica, 46, 33-50. 36

Koenker, R. W. (2005), Quantile Regression, Cambridge, UK: CambridgeUniversity Press. 47

Kuan, C., Yeh, J. and Hsu, Y. (2009) Assessing Value at Risk with CARE, the Conditional Autoregressive Expectile Models, Journal of Econometrics, 150, 261270. $2,45,50,97,100,110$

Kuester, K., Mittnik, S. and Paolella, M.,S. (2006), Value-at-Risk Prediction: A Comparison of Alternative Strategies, Journal of Financial Econometrics, 4, 5389. 31,34

Lambert, P. and Laurent, S. (2002), Modelling Skewness Dynamics in Series of Financial Data Using Skewed Location-Scale Distributions, Institut de Statistique, Université Catholique de Louvain, Louvain-la-Neuve, Belgium, Discussion Paper No. 01-25. 14

McNeil, A. J. and Saladin, T. (1997), The Peaks of Thresholds Method for Estimating High Quantiles of Loss Distributions, In: Proceeding of XXVIIth International ASTIN, Colloquium, Cairns, p. 23-43. 24

McNeil, A. J. and Frey, R. (2000), Estimation of Tail-Related Risk Measures for Heteroscedastic Financial Time Series: an Extreme Value Approach, Journal of Empirical Finance, 7, 271-300. 70

McNeil, A. J., Frey, R. and Embrechts, P. (2005), Quantitative Risk Management, New Jersey: Princeton University Press. 61, 63, 115

Morettin, P. A. (2008), Econometria Financeira, segunda Ed., São Paulo. 1, 5

Newey, W. K. and Powell, J.L. (1987), Asymmetric Least Squares Estimation and Testing, Econometrica, 55, 819-847. 53, 54, 57 
Taylor, J. W. (2008), Estimating Value at Risk and Expected Shortfall Using Expectiles, Journal of Financial Econometrics, 6, 231-252. 2, 45, 48, 49, 50, 66, 70, 90, 97, 98, 104, 110

Tsay, R. S. (2005), Analysis of Financial Time Series, Wiley-Interscience, second Ed., New Jersey. 27

Weiss, A. (1991), Estimating Nonlinear Dynamic Models Using Least Absolute Error Estimation, Econometric Theory, 7, 46-68. 39

Zivot, W. and Wang, J. (2006), Modeling Financial Time Series With S-Plus, Springer, second Ed., New York. 23, 27 